\title{
FROM MOTIVES TO COMODULES OVER THE MOTIVIC HOPF ALGEBRA
}

\author{
JOSEPH AYOUB
}

To Charles Weibel for his 65th anniversary

\begin{abstract}
We construct the natural functor from the triangulated category of motives (over a field endowed with a complex embedding) to the triangulated category of homotopy comodules over the motivic homotopy Hopf algebra.

Keywords: homotopy Hopf algebra, homotopy comodule, motive, motivic Hopf algebra.
\end{abstract}

AMS 2010 Mathematics subject classification: 14F42; 18D10; 18G30; 18G55; $19 \mathrm{E} 15$.

\section{CONTENTS}

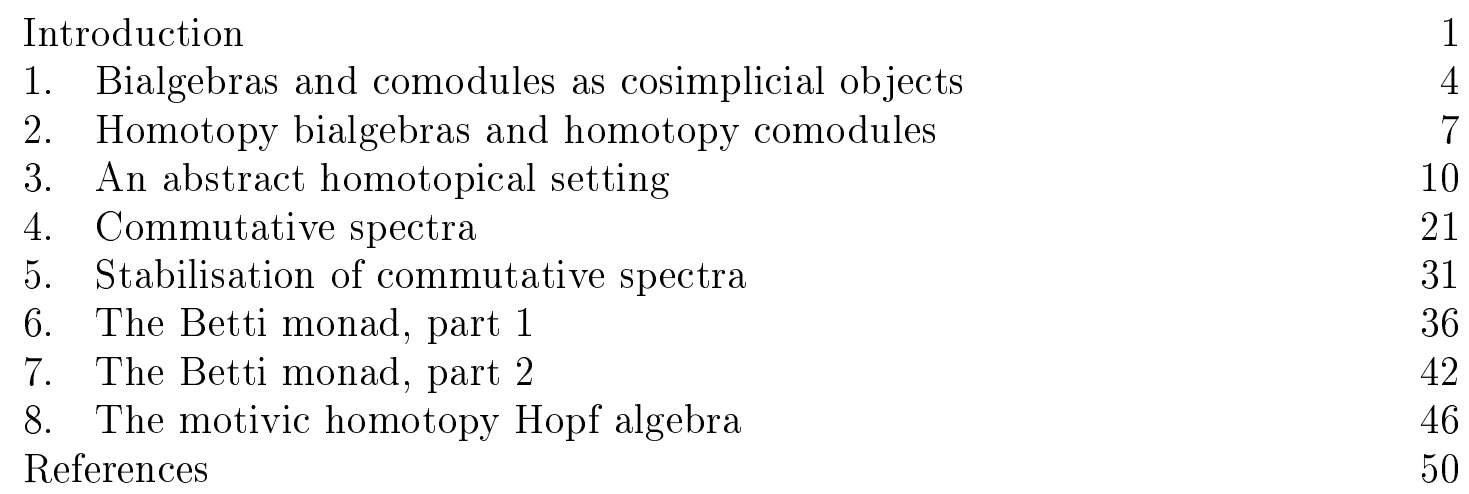

Introduction. This paper complements [5, 6] where the motivic Hopf algebra $\mathcal{H}_{\text {mot }}(k, \sigma ; \Lambda)$ of a field $k$ endowed with a complex embedding $\sigma: k \hookrightarrow \mathbb{C}$ was constructed and studied. (As usual, $\Lambda$ denotes the ring of coefficients.) Recall that, given a $k$-motive $M$, its Betti realisation $\operatorname{Bti}_{\sigma}^{*}(M)$ is naturally a comodule over $\mathcal{H}_{\text {mot }}(k, \sigma ; \Lambda)$. This yields a functor

$$
\mathbf{D A}^{\text {ét }}(k ; \Lambda) \longrightarrow \operatorname{coMod}\left(\mathcal{H}_{\text {mot }}(k, \sigma ; \Lambda)\right)
$$

from the category of motives (étale and with coefficients in $\Lambda$ ) to the category of comodules over $\mathcal{H}_{\text {mot }}(k, \sigma ; \Lambda)$.

Unfortunately, there are obvious drawbacks: $\mathcal{H}_{\text {mot }}(k, \sigma ; \Lambda)$ is only a Hopf algebra in $\mathbf{D}(\Lambda)$, the derived category of $\Lambda$-modules, and, similarly, the coaction of $\mathcal{H}_{\text {mot }}(k, \sigma ; \Lambda)$ on $\operatorname{Bti}_{\sigma}^{*}(M)$ is only defined in $\mathbf{D}(\Lambda)$. In particular, the target of the functor (1) is not a triangulated category.

The author was supported in part by the Swiss National Science Foundation (NSF), Grant No. 200021-144372/1. 
It is natural to expect that $\mathcal{H}_{\text {mot }}(k, \sigma ; \Lambda)$ can be enhanced into a motivic homotopy Hopf algebra $\underline{\mathcal{H}}_{\text {mot }}(k, \sigma ; \Lambda)$ and that $\operatorname{Bti}_{\sigma}^{*}(M)$ can be enhanced into a homotopy comodule over $\underline{\mathcal{H}}_{\text {mot }}(k, \sigma ; \Lambda) .1$ This would then yield a triangulated functor

$$
\mathbf{D A}^{\text {ét }}(k ; \Lambda) \longrightarrow \operatorname{hocoMod}\left(\underline{\mathcal{H}}_{\text {mot }}(k, \sigma ; \Lambda)\right)
$$

that refines (1). The goal of this paper is precisely to carry out this expectation.

Relation with the work of Nori. The motivic Hopf algebra $\mathcal{H}_{\text {mot }}(k, \sigma ; \Lambda)$ is acyclic in strictly negative homological degrees and thus its zeroth homology $\mathbf{H}_{\text {mot }}(k, \sigma ; \Lambda)$ is naturally a Hopf algebra in the category of $\Lambda$-modules. By the work of Nori [12] and thanks to the comparison result of [7], one has a triangulated functor

$$
\mathbf{D A}^{\text {ét }}(k ; \Lambda) \longrightarrow \mathbf{D}\left(\operatorname{coMod}\left(\mathbf{H}_{\text {mot }}(k, \sigma ; \Lambda)\right)\right)
$$

where $\operatorname{coMod}\left(\mathbf{H}_{\text {mot }}(k, \sigma ; \Lambda)\right)$ is the abelian category of comodules over $\mathbf{H}_{\text {mot }}(k, \sigma ; \Lambda)$ in the category of $\Lambda$-modules. (For details about the construction of (3), see [7, $\S 7.4]$.

Considering $\mathbf{H}_{\text {mot }}(k, \sigma ; \Lambda)$ as a homotopy Hopf algebra, one also has the triangulated category hocoMod $\left(\mathbf{H}_{\text {mot }}(k, \sigma ; \Lambda)\right)$ of homotopy comodules over $\mathbf{H}_{\text {mot }}(k, \sigma ; \Lambda)$. Assuming that $\Lambda$ is a field, this category is closely related to the derived category of the abelian category of comodules over $\mathbf{H}_{\text {mot }}(k, \sigma ; \Lambda)$. Indeed, there is a natural $t$-structure on hocoMod $\left(\mathbf{H}_{\operatorname{mot}}(k, \sigma ; \Lambda)\right)$ whose heart is exactly $\operatorname{coMod}\left(\mathbf{H}_{\operatorname{mot}}(k, \sigma ; \Lambda)\right)$. Moreover, the obvious functor

$$
\mathbf{D}\left(\operatorname{coMod}\left(\mathbf{H}_{\operatorname{mot}}(k, \sigma ; \Lambda)\right)\right) \longrightarrow \operatorname{hocoMod}\left(\mathbf{H}_{\operatorname{mot}}(k, \sigma ; \Lambda)\right)
$$

is $t$-exact, fully faithful, commutes with homotopy colimits and induces an equivalence when restricted to the subcategories of objects which are $t$-bounded from the left.

There is a corestriction functor ${ }^{2}$

$$
\operatorname{hocoMod}\left(\underline{\mathcal{H}}_{\operatorname{mot}}(k, \sigma ; \Lambda)\right) \longrightarrow \operatorname{hocoMod}\left(\mathbf{H}_{\operatorname{mot}}(k, \sigma ; \Lambda)\right),
$$

and the composed functor (5) $\circ(2): \mathbf{D A}^{\text {ét }}(k ; \Lambda) \longrightarrow \operatorname{hocoMod}\left(\mathbf{H}_{\text {mot }}(k, \sigma ; \Lambda)\right)$ factors through the fully faithful embedding (4). (This follows from the properties of (4) mentioned above and the fact that $\mathbf{D} \mathbf{A}^{\mathrm{et}}(k ; \Lambda)$ is compactly generated by motives whose image by (5) $\circ(2)$ are $t$-bounded.) This gives a functor from $\mathbf{D} A^{\text {ét }}(k ; \Lambda)$

\footnotetext{
${ }^{1}$ Informally speaking, the multiplication of a homotopy Hopf algebra is strict whereas its comultiplication is lax (i.e., the comultiplication is only defined up to homotopy and the coassociativity property is only satisfied up to coherent higher homotopies). Similarly, the coaction of a homotopy Hopf algebra on a homotopy comodule is lax. For the precise notions we refer the reader to Definitions 2.1 and 2.3 .

${ }^{2}$ This functor is constructed as follows. Consider the diagram of cosimplicial algebras

$$
\underline{\mathcal{H}}_{\text {mot }}(k, \sigma ; \Lambda) \longleftarrow \tau_{\geqslant 0}\left(\underline{\mathcal{H}}_{\text {mot }}(k, \sigma ; \Lambda)\right) \longrightarrow \mathrm{H}_{0}\left(\underline{\mathcal{H}}_{\text {mot }}(k, \sigma ; \Lambda)\right) .
$$
}

The left arrow is a quasi-isomorphism by [5, Corollaire 2.105] which implies also that

$$
\mathrm{H}_{0}\left(\underline{\mathcal{H}}_{\operatorname{mot}}(k, \sigma ; \Lambda)\right)=\mathrm{B}\left(\mathbf{H}_{\text {mot }}(k, \sigma ; \Lambda)\right)
$$

where $\mathrm{B}(-)$ is as in Construction 1.1. Thus, the above diagram is actually a diagram of homotopy Hopf algebras where the left arrow is a quasi-isomorphism. Applying Proposition 2.12, we deduce two triangulated functors

$$
\operatorname{hocoMod}\left(\underline{\mathcal{H}}_{\operatorname{mot}}(k, \sigma ; \Lambda)\right) \longleftarrow \operatorname{hocoMod}\left(\tau \geqslant 0\left(\underline{\mathcal{H}}_{\operatorname{mot}}(k, \sigma ; \Lambda)\right)\right) \longrightarrow \operatorname{hocoMod}\left(\mathbf{H}_{\text {mot }}(k ; \sigma ; \Lambda)\right)
$$

where the left one is an equivalence. This gives the functor (5). 
to $\mathbf{D}\left(\operatorname{coMod}\left(\mathbf{H}_{\text {mot }}(k, \sigma ; \Lambda)\right)\right)$ which we expect to coincide with (3) up to an isomorphism. We also expect this compatibility to be routine but lengthy, and we do not attempt to verify it here. Finally, recall that $\underline{\mathcal{H}}_{\text {mot }}(k, \sigma ; \Lambda)$ is conjectured to be acyclic except in degree zero (see [5, page 126, Conjecture A]). Therefore, $\underline{\mathcal{H}}_{\text {mot }}(k, \sigma ; \Lambda)$ is conjecturally quasi-isomorphic to $\mathbf{H}_{\text {mot }}(k, \sigma ; \Lambda)$, so that the functor (5) is conjecturally an equivalence of categories and the functors (2) and (3) are conjecturally the same (up to the fully faithful embedding (4)).

Relation with the work of Pridham. By [13], $\mathcal{H}_{\text {mot }}(k, \sigma ; \Lambda)$ can be enhanced into a motivic dg Hopf algebra. Pridham's work also gives a functor similar to (2) but where the target is the homotopy category of the dg category of dg comodules over the motivic dg Hopf algebra. In a sense that I don't really comprehend yet, Pridham's motivic dg Hopf algebra is more strict than our motivic homotopy Hopf algebra. This is illustrated by the fact that in [13], Pridham uses a stronger notion of equivalence between dg Hopf algebras. Indeed, in loc. cit., a morphism of $\mathrm{dg}$ Hopf algebras inducing a quasi-isomorphism on the underlying complexes does not necessarily induce an equivalence on the dg categories of dg comodules (whereas, with our definitions, a morphism of homotopy Hopf algebras inducing a quasi-isomorphism on the underlying complexes does induce an equivalence on the categories of homotopy comodules).

Also, it should be noted that our approach is much more explicit than Pridham's [13] and uses heavily the specificity of the situation (contrary to the approach of Pridham which is applicable to general dg categories). In particular, our method gives a nice and concrete model for the motivic homotopy Hopf algebra. Nevertheless, a serious comparison with Pridham's work should be done, but this probably deserves a separate paper.

Organisation. In Section 1, we recall well known interpretations of bialgebras and comodules as certain cosimplicial objects. In Section 2, we recall the notions of "homotopy Hopf algebras" and "homotopy comodules". In Section 3, we describe a situation which naturally gives rise to a homotopy Hopf algebra. In Section 4. we introduce the notion of commutative spectra which is a variant of the notion of symmetric spectra. In Section 5, we discuss the stabilisation of commutative spectra. In Sections 6 and 7, we recall and complement the explicit model of the monad $\mathrm{Bti}_{\sigma, *} \mathrm{Bti}_{\sigma}^{*}$ obtained in [5, §2.2.5]. In Section 8, we construct the promised enhancements and the functor (2).

Notation. We work over a base field $k$ of characteristic zero endowed with a complex embedding $\sigma: k \hookrightarrow \mathbb{C}$. By $k$-variety we mean a separated $k$-scheme of finite type. We denote by $\mathrm{Sch} / k$ the category of $k$-varieties and by $\mathrm{Sm} / k$ its subcategory of smooth $k$-varieties. Given a $k$-variety $X$, we denote by $X^{\text {an }}$ the associated complex analytic space (which depends on $\sigma$ ).

We fix a commutative ring of coefficients $\Lambda$. For most of the constructions, it will be necessary to assume that $\Lambda$ is a $\mathbb{Q}$-algebra. We denote by $\operatorname{Cpl}(\Lambda)$ the category of complexes of $\Lambda$-modules and by $\mathbf{D}(\Lambda)$ the derived category. More generally, given an additive category $\mathcal{A}$, we denote by $\operatorname{Cpl}(\mathcal{A})$ the category of complexes in $\mathcal{A}$ and, if $\mathcal{A}$ is abelian, we denote by $\mathbf{D}(\mathcal{A})$ its derived category.

Given a model category $\mathfrak{M}$, we denote by $\operatorname{Ho}(\mathfrak{M})$ its homotopy category. Given an essentially small category $\mathcal{C}$, we denote by $\operatorname{PSh}(\mathcal{C} ; \Lambda)$ the category of presheaves 
of $\Lambda$-modules on $\mathcal{C}$. If $\mathcal{C}$ comes with a Grothendieck topology $\tau, \mathbf{C p l}(\mathbf{P S h}(\mathcal{C} ; \Lambda))$ has a $\tau$-local model structure and we denote by $\mathbf{H o}_{\tau}(\mathbf{C p l}(\mathbf{P S h}(\mathcal{C} ; \Lambda)))$ its homotopy category.

As usual, we denote by $\boldsymbol{\Delta}$ the category of simplices (aka., finite ordinals $[n]=$ $\{0 \leqslant \cdots \leqslant n\}$.) A simplicial (resp. cosimplicial) object is a contravariant (resp. covariant) functor from $\Delta$. Given a category $\mathcal{E}$, we denote by $\Delta^{\mathrm{op}} \mathcal{E}$ (resp. $\Delta \mathcal{E}$ ) the category of simplicial (resp. cosimplicial) objects of $\mathcal{E}$.

\section{BialgeBras AND COMODUles As COSIMPliCIAL OBJECTS}

In this section, we fix a symmetric monoidal category with unit $(\varepsilon, \otimes, \mathbf{1})$. If not otherwise stated, algebras in $\mathcal{E}$ will be commutative and unital. Coalgebras in $\mathcal{E}$ will be counital but not necessary cocommutative. (See [5, §1.1] for more details.) The following construction is well known; it is dual to the construction of the classifying simplicial set of a monoid (see Remark 1.2 below).

Construction 1.1 — Let $A$ be a bialgebra in $\mathcal{E}$. We associate to $A$ a cosimplicial algebra $\mathrm{B}(A)$ in $\mathcal{E}$ as follows.

(1) For $n \in \mathbb{N}$, we set

$$
\mathrm{B}^{n}(A)=\overbrace{A \otimes \cdots \otimes A}^{n \text { times }} .
$$

(2) For $0 \leqslant i \leqslant n$, the map $\sigma_{i}: \mathrm{B}^{n+1}(A) \longrightarrow \mathrm{B}^{n}(A)$ is given by

$$
A^{\otimes n+1}=A^{\otimes i} \otimes A \otimes A^{\otimes n-i} \stackrel{\mathrm{id} \otimes \mathrm{cu} \otimes \mathrm{id}}{\longrightarrow} A^{\otimes i} \otimes \mathbf{1} \otimes A^{\otimes n-i}=A^{\otimes n} .
$$

(3) The map $\delta_{0}: \mathrm{B}^{n}(A) \longrightarrow \mathrm{B}^{n+1}(A)$ is given by

$$
A^{\otimes n}=\mathbf{1} \otimes A^{\otimes n} \stackrel{\mathrm{u} \otimes \mathrm{id}}{\longrightarrow} A \otimes A^{\otimes n}=A^{\otimes n+1} .
$$

(4) For $1 \leqslant i \leqslant n$, the map $\delta_{i}: \mathrm{B}^{n}(A) \longrightarrow \mathrm{B}^{n+1}(A)$ is given by

$$
A^{\otimes n}=A^{\otimes i-1} \otimes A \otimes A^{\otimes n-i} \stackrel{\text { id } \otimes \mathrm{cm} \otimes \mathrm{id}}{\longrightarrow} A^{\otimes i-1} \otimes A \otimes A \otimes A^{\otimes n-i}=A^{\otimes n+1} .
$$

(5) The map $\delta_{n+1}: \mathrm{B}^{n}(A) \longrightarrow \mathrm{B}^{n+1}(A)$ is given by

$$
A^{\otimes n}=A^{\otimes n} \otimes 1 \stackrel{\mathrm{id} \otimes \mathrm{u}}{\longrightarrow} A^{\otimes n} \otimes A=A^{\otimes n+1} .
$$

Of course, $\mathrm{u}, \mathrm{cu}$ and $\mathrm{cm}$ denote the unit, counit, and comultiplication of $A$.

Remark 1.2 - Keep the notation as in Construction 1.1. Let $E$ be an algebra in $\mathcal{E}$. Then the set of algebra homomorphisms $\operatorname{hom}_{\mathrm{Alg}}(A, E)$ is naturally a monoid. Moreover, it is easy to see that the simplicial set $\operatorname{hom}_{\mathrm{Alg}}(\mathrm{B}(A), E)$ identifies with the classifying simplicial set of the monoid $\operatorname{hom}_{\mathrm{Alg}}(A, E)$ which is given in degree $n \in \mathbb{N}$ by the set of functors

$$
[n]^{\text {op }} \longrightarrow \bullet_{\operatorname{hom}_{\mathrm{Alg}}(A, E)}
$$

where, for a monoid $M, \bullet_{M}$ is the category with one object $\bullet$ satisfying end $(\bullet)=M$. By the Yoneda lemma, this gives a quick verification that Construction 1.1 yields indeed a cosimplicial algebra.

Definition $\mathbf{1 . 3}$ - Let $B$ be a cosimplicial algebra in $\mathcal{E}$. We introduce three conditions on $B$.

(B1) The unit map $\mathrm{u}: \mathbf{1} \longrightarrow B^{0}$ is an isomorphism. 
(B2) For every $n \geqslant 1$, the natural map

$$
\eta_{1} \cdots \eta_{n}: \overbrace{B^{1} \otimes \cdots \otimes B^{1}}^{n \text { times }} \longrightarrow B^{n},
$$

induced by the maps $\eta_{i}:[1] \rightarrow[n]$ given by $\eta_{i}(0)=i-1$ and $\eta_{i}(1)=i$, is an isomorphism.

(B3) The natural maps

$$
\begin{aligned}
& \quad \delta_{1} \cdot \delta_{0}: B^{1} \otimes B^{1} \longrightarrow B^{2} \quad \text { and } \quad \delta_{2} \cdot \delta_{1}: B^{1} \otimes B^{1} \longrightarrow B^{2} \\
& \text { are isomorphisms }\left.\right|^{3}
\end{aligned}
$$

The following result is well known.

Proposition 1.4 - The functor $A \mapsto \mathrm{B}(A)$ gives an equivalence of categories between the category of bialgebras in $\mathcal{E}$ and the category of cosimplicial algebras in $\mathcal{E}$ satisfying the conditions (B1) and (B2) of Definition 1.3. Moreover, a bialgebra $A$ is a Hopf algebra if and only if $\mathrm{B}(A)$ satisfies the condition (B3) of Definition 1.3.

Proof. The fact that $\mathrm{B}(A)$ satisfies the conditions (B1) and (B2) is clear. Similarly, if $A$ is a Hopf algebra, it is easy to see that $\mathrm{B}(A)$ satisfies the condition (B3).

Now, let $B$ be a cosimplicial algebra satisfying (B1) and (B2). We will show that $B^{1}$ is naturally a bialgebra. We define the comultiplication $\mathrm{cm}: B^{1} \longrightarrow B^{1} \otimes B^{1}$ by the composition of

$$
B^{1} \stackrel{\delta_{1}}{\longrightarrow} B^{2} \stackrel{\delta_{2} \cdot \delta_{0}}{\sim} B^{1} \otimes B^{1} .
$$

To check that this comultiplication is coassociative, we identify the two possible maps with the composition of

$$
B^{1} \stackrel{\eta}{\longrightarrow} B^{3} \stackrel{\eta_{1} \cdot \eta_{2} \cdot \eta_{3}}{\sim} B^{1} \otimes B^{1} \otimes B^{1}
$$

where the first morphism is induced by $\eta:[1] \rightarrow[3]$ sending 0 and 1 to 0 and 3 respectively. The remaining verifications are easy and will be omitted.

Alternatively, one could use the Yoneda lemma as in Remark 1.2 to reduce to the classical dual statement about monoids and their classifying simplicial sets.

Construction 1.5 - Let $A$ be a bialgebra in $\mathcal{E}$ and $L$ a left comodule over $A$. We associate to $L$ a $\mathrm{B}(A)$-module $\mathrm{M}(L)$ as follows.

(1) For $n \in \mathbb{N}$, we set

$$
\mathrm{M}^{n}(L)=\mathrm{B}^{n}(A) \otimes L=\overbrace{A \otimes \cdots \otimes A}^{n \text { times }} \otimes L .
$$

(2) For $0 \leqslant i \leqslant n$, the map $\sigma_{i}: \mathrm{M}^{n+1}(L) \longrightarrow \mathrm{M}^{n}(L)$ is given by

$$
\mathrm{B}^{n+1}(A) \otimes L \stackrel{\sigma_{i} \otimes \mathrm{id}}{\longrightarrow} \mathrm{B}^{n}(A) \otimes L .
$$

(3) For $0 \leqslant i \leqslant n$, the map $\delta_{i}: \mathrm{M}^{n}(L) \longrightarrow \mathrm{M}^{n+1}(L)$ is given by

$$
\mathrm{B}^{n}(A) \otimes L \stackrel{\delta_{i} \otimes \mathrm{id}}{\longrightarrow} \mathrm{B}^{n+1}(A) \otimes L .
$$

(4) The map $\delta_{n+1}: \mathrm{M}^{n}(L) \longrightarrow \mathrm{M}^{n+1}(L)$ is given by

$$
A^{\otimes n} \otimes L \stackrel{\text { id } \otimes \mathrm{ca}}{\longrightarrow} A^{\otimes n} \otimes A \otimes L=A^{\otimes n+1} \otimes L .
$$

\footnotetext{
${ }^{3}$ Note that, with the notation of (B2), we have $\delta_{0}=\eta_{2}$ and $\delta_{2}=\eta_{1}$.
} 
Of course, ca denotes the coaction of $A$ on $L$.

Similarly, given a right comodule $R$ over $A$, we may construct a $\mathrm{B}(A)$-module $\mathrm{M}(R)$ given by $\mathrm{M}^{n}(R)=R \otimes \mathrm{B}^{n}(A)$, etc.

DEFINITION 1.6 - Let $B$ be a cosimplicial algebra in $\mathcal{E}$ satisfying the condition (B1) of Definition 1.3. Let $M$ be a B-module. We introduce two conditions on $M$.

(Cl) For all $n \in \mathbb{N}$, the morphism of $B^{n}$-modules

$$
B^{n} \otimes M^{0} \longrightarrow M^{n}
$$

induced by the map $\iota_{n}:[0] \rightarrow[n]$ given by $\iota_{n}(0)=n$, is an isomorphism.

(Cr) For all $n \in \mathbb{N}$, the morphism of $B^{n}$-modules

$$
M^{0} \otimes B^{n} \longrightarrow M^{n}
$$

induced by the map $\iota_{0}:[0] \rightarrow[n]$ given by $\iota_{0}(0)=0$, is an isomorphism.

The following result is well known.

Proposition 1.7 - Let $A$ be a bialgebra in $\mathcal{E}$. The functor $L \mapsto \mathrm{M}(L)$ (resp. $R \mapsto \mathrm{M}(R)$ ) gives an equivalence of categories between the category of left (resp. right) comodules over $A$ and the category of $\mathrm{B}(A)$-modules satisfying the conditions (Cl) (resp. (Cr)) of Definition 1.6.

Proof. We only discuss the case of left comodules. Clearly, given a left comodule $L$ over $A$, the $\mathrm{B}(A)$-module $\mathrm{M}(L)$ satisfies the condition $(\mathrm{Cl})$.

Conversely, let $M$ be a $\mathrm{B}(A)$-module satisfying $(\mathrm{Cl})$. We define the coaction of $A$ on $M^{0}$ by the composition of

$$
M^{0} \stackrel{\iota_{0}}{\longrightarrow} M^{1} \stackrel{\iota_{1}}{\sim} A \otimes M^{0}
$$

To check that this coaction is coassociative, we identify the two possible maps with the composition of

$$
M^{0} \stackrel{\iota_{0}}{\longrightarrow} M^{2} \stackrel{\iota_{2}}{\sim} \mathrm{B}^{2}(A) \otimes M^{0}
$$

The remaining verifications are easy and will be omitted.

Proposition 1.8 - Let $B$ be a cosimplicial algebra in $\mathcal{E}$ satisfying the conditions (B1), (B2) and (B3) of Definition 1.3. Let $M$ be a B-module and assume that $M$ satisfies condition (Cl) (resp. (Cr)) of Definition 1.6. Then, $M$ satisfies the following stronger condition.

(Cs) For all $0 \leqslant m \leqslant n$, the morphism of $B^{n}$-modules

$$
B^{n} \otimes M^{0} \longrightarrow M^{n}
$$

induced by the map $\iota_{m}:[0] \rightarrow[n]$ given by $\iota_{m}(0)=m$, is an isomorphism.

In particular, $M$ satisfies condition $(C r)$ (resp. (Cl)) of Definition 1.6.

Proof. By Propositions 1.4 and 1.7, we may assume that $B=\mathrm{B}(A)$ and $M=\mathrm{M}(L)$ where $A$ is a Hopf algebra and $L$ is a left comodule over $A$. The claim is then easy and the details will be omitted. 


\section{Homotopy BiAlgeBRAS AND Homotopy COMODUleS}

In this section, we fix a symmetric monoidal model category with unit $(\mathfrak{E}, \otimes, \mathbf{1})$ in the sense of Hovey [9, Chapter 4]; in fact, for simplicity, we will assume that the unit object 1 is cofibrant as in [2, Définition 4.1.57]. We are mostly interested in the case where $\mathfrak{E}=\operatorname{Cpl}(\Lambda)$; in this case "algebras" are usually called "dg algebras".

The following definition is motivated by the considerations in Section 1. We have learned this definition from B. Toën. Clearly, it is also a variant of the concept of a Segal space (see [14]).

Definition 2.1 - Let $B$ be a cosimplicial algebra in $\mathfrak{E}$. We say that $B$ is a homotopy bialgebra if the following two conditions are satisfied.

(hB1) The unit map $\mathrm{u}: 1 \longrightarrow B^{0}$ is a weak equivalence.

(hB2) For every $n \geqslant 1$, the natural map

$$
\eta_{1} \cdots \eta_{n}: \overbrace{B^{1} \otimes \cdots \otimes B^{1}}^{n \text { times }} \longrightarrow B^{n},
$$

induced by the maps $\eta_{i}:[1] \rightarrow[n]$ given by $\eta_{i}(0)=i-1$ and $\eta_{i}(1)=i$, is a weak equivalence.

We say that $B$ is a homotopy Hopf algebra if moreover the following condition is satisfied.

(hB3) The natural maps

$$
\delta_{1} \cdot \delta_{0}: B^{1} \stackrel{\mathrm{L}}{\otimes} B^{1} \longrightarrow B^{2} \quad \text { and } \quad \delta_{2} \cdot \delta_{1}: B^{1} \stackrel{\mathrm{L}}{\otimes} B^{1} \longrightarrow B^{2}
$$

are weak equivalences. ${ }^{4}$

Remark 2.2 - Clearly, a cosimplical algebra $B$ is a homotopy bialgebra (resp. a homotopy Hopf algebra) if and only if $B$, considered as a cosimplicial algebra in $\mathbf{H o}(\mathfrak{E})$, satisfies the conditions (B1) and (B2) (resp. (B1)-(B3)) of Definition 1.3. In particular, by Proposition 1.4 $B$ determines a bialgebra (resp. a Hopf algebra) in the monoidal category $\mathbf{H o}(\mathfrak{E})$.

DeFinition 2.3 - Let $B$ be a homotopy bialgebra and let $M$ be a B-module. We say that $M$ is a left homotopy comodule over $B$ if the following condition is satisfied.

( $h C l$ ) For all $n \in \mathbb{N}$, the natural map

$$
B^{n} \stackrel{\mathrm{L}}{\otimes} M^{0} \longrightarrow M^{n}
$$

induced by the action of $B^{n}$ on $M^{n}$ and the map $\iota_{n}:[0] \rightarrow[n]$ given by $\iota_{n}(0)=n$, is a weak equivalence.

Similarly, we say that $M$ is a right homotopy comodule over $B$ if the following condition is satisfied.

( $h \mathrm{Cr}$ ) For all $n \in \mathbb{N}$, the natural map

$$
M^{0} \stackrel{\mathrm{L}}{\otimes} B^{n} \longrightarrow M^{n}
$$

induced by the action of $B^{n}$ on $M^{n}$ and the map $\iota_{0}:[0] \rightarrow[n]$ given by $\iota_{0}(0)=0$, is a weak equivalence.

\footnotetext{
${ }^{4}$ Note that, with the notation of (hB2), we have $\delta_{0}=\eta_{2}$ and $\delta_{2}=\eta_{1}$.
} 
Morphisms between left (resp. right) homotopy comodules over $B$ are simply morphisms of B-modules.

Remark 2.4 - Let $B$ be a homotopy bialgebra. Clearly, a $B$-module $M$ is a left (resp. right) homotopy comodule over $B$ if and only if $M$, considered as a cosimplicial object in $\mathbf{H o}(\mathfrak{E})$, satisfies condition $(\mathrm{Cl})$ (resp. $(\mathrm{Cr})$ ) of Definition 1.6. In particular, by Propositions 1.4 and 1.7, $M$ determines a left (resp. right) comodule $M^{0}$ over the bialgebra $B^{1}$ in $\mathbf{H o}(\mathfrak{E})$.

For later use, we record the following observation. (We note that the class of weak equivalences in $\boldsymbol{\Delta} \mathfrak{M}$ consists of those maps which are weak equivalences in every cosimplicial degree.)

LEMMA $2.5-$ Let $B$ be a homotopy bialgebra and let $M \longrightarrow M^{\prime}$ be a morphism of left (resp. right) homotopy comodules over B. Then the following conditions are equivalent:

(i) $M^{0} \longrightarrow M^{\prime 0}$ is a weak equivalence in $\mathfrak{M}$;

(ii) $M \longrightarrow M^{\prime}$ is a weak equivalence in $\Delta \mathfrak{M}$.

Proposition 2.6 - Let $M$ be a left (resp. right) homotopy comodule over a homotopy bialgebra B. Assume that $B$ is a homotopy Hopf algebra. Then, $M$ satisfies the following condition.

(hCs) For all $0 \leqslant m \leqslant n$, the natural map

$$
B^{n} \stackrel{\mathrm{L}}{\otimes} M^{0} \longrightarrow M^{n},
$$

induced by the action of $B^{n}$ on $M^{n}$ and the map $\iota_{m}:[0] \rightarrow[n]$ given by $\iota_{m}(0)=m$, is a weak equivalence.

In particular, $M$ is also a right (resp. left) homotopy comodule over $B$.

Proof. This follows immediately from Proposition 1.8 .

In the remainder of this section, we will use results from [15]. For this, we need to impose some mild technical assumptions on the monoidal model category $\mathfrak{E}$. Namely, we will assume the following.

\section{Hypothesis 2.7}

- The model category $\mathfrak{E}$ is presentable by cofibrations as in [2, Définition 4.2.39]. (Another possibility is to assume that $\mathfrak{E}$ is cellular as in [8, Definition 12.1.1].)

- The monoidal model category $\mathfrak{E}$ satisfies the monoid axiom as in [15, Definition 3.3].

Remark 2.8 - The assumption that $\mathfrak{E}$ is presentable by cofibrations (or cellular) insures that $\mathfrak{E}$ is cofibrantly generated as in [15, Definition 2.2]. Clearly, $\mathbf{C p l}(\Lambda)$, endowed with the projective model structure, satisfies both assumptions in Hypothesis 2.7

Proposition $2.9-$ Let $B$ be a cosimplicial algebra in $\mathfrak{E}$. Then $\operatorname{Mod}(B)$ is naturally a monoidal model category. Weak equivalences and fibrations in $\operatorname{Mod}(B)$ are preserved and detected by the forgetful functor from $\operatorname{Mod}(B)$ to the category of cosimplical objets in $\mathfrak{E}$ endowed with its injective model structure (see [2, Définition 4.4.15]). 
Proof. The category $\Delta \mathfrak{E}$ has an injective model structure (see [2, Proposition 4.4.17]) where cofibrations and weak equivalences are defined degreewise. In fact, $\Delta \mathfrak{E}$ is a monoidal model category satisfying the assumptions in Hypothesis 2.7. The claim now follows from [15, Theorem 4.1(2)].

DEFINITION 2.10 - Let $B$ be a homotopy bialgebra. We denote by $\operatorname{hocoMod}_{l}(B)$ $\left(\right.$ resp. $\left.\operatorname{hocoMod}_{r}(B)\right)$ the full subcategory of $\mathbf{H o}(\operatorname{Mod}(B))$ consisting of left (resp. right) homotopy comodules over $B$. If $B$ is a homotopy Hopf algebra, the categories $\operatorname{hocoMod}_{l}(B)$ and $\operatorname{hocoMod}_{r}(B)$ coincide by Proposition 2.6, and we simply write $\operatorname{hocoMod}(B)$.

We end this section with a few functoriality results.

Proposition 2.11 - We assume that the endofunctor $Q \otimes-$ of $\mathfrak{E}$ preserves weak equivalences for every cofibrant object $Q \in \mathfrak{E}$. Let $B$ be a homotopy bialgebra in $\mathfrak{E}$. Then

$$
\operatorname{hocoMod}_{l}(B) \subset \mathbf{H o}(\operatorname{Mod}(B)) \quad \text { and } \operatorname{hocoMod}_{r}(B) \subset \mathbf{H o}(\operatorname{Mod}(B))
$$

are monoidal subcategories. Moreover, if $\mathfrak{E}$ is stable (see [9, Definition 7.1.1] or [2, Définition 4.1.44]), these are also triangulated subcategories.

Proof. Let $M$ and $N$ be two left homotopy comodules over $B$. We may assume that $M$ and $N$ are cofibrant objects in $\operatorname{Mod}(B)$. We need to show that $M \otimes_{B} N$ is still a left homotopy comodule, i.e., we need to show that, for $n \in \mathbb{N}$, the map

$$
B^{n} \stackrel{\mathrm{L}}{\otimes}\left(M^{0} \otimes_{B^{0}} N^{0}\right) \longrightarrow M^{n} \otimes_{B^{n}} N^{n},
$$

induced by $\iota_{n}:[0] \rightarrow[n]$, is a weak equivalence.

Let $U \longrightarrow M^{0}$ and $V \longrightarrow N^{0}$ be cofibrant replacements. As $1 \longrightarrow B^{0}$ is a weak equivalence, we may use [15, Theorem 4.3] to deduce that the natural map

$$
U \otimes V \longrightarrow M^{0} \otimes_{B^{0}} N^{0}
$$

is a weak equivalence; this already uses the extra assumption on $\mathfrak{E}$. Using again this assumption, we get weak equivalences

$$
\begin{gathered}
B^{n} \stackrel{\mathrm{L}}{\otimes}(U \otimes V) \longrightarrow B^{n} \otimes(U \otimes V), \\
B^{n} \stackrel{\mathrm{L}}{\otimes} M^{0} \longrightarrow B^{n} \otimes U \quad \text { and } \quad B^{n} \stackrel{\mathrm{L}}{\otimes} N^{0} \longrightarrow B^{n} \otimes V .
\end{gathered}
$$

Thus, we are left to show that

$$
B^{n} \otimes(U \otimes V) \longrightarrow\left(B^{n} \otimes U\right) \otimes_{B^{n}}\left(B^{n} \otimes V\right)
$$

is a weak equivalence. But the latter is an isomorphism, and we are done.

Next, assume that $\mathfrak{E}$ is stable; it follows that $\operatorname{Mod}(B)$ is also stable. It is clear that the subcategory hocoMod $(B)$ is closed under homotopy pushout. Thus, it remains to show that this subcategory is closed under the quasi-inverse of the suspension functor $\Sigma^{1}$. But this quasi-inverse is given by $-\stackrel{\mathrm{L}}{\otimes}_{B} \underline{\operatorname{Hom}}\left(\Sigma^{1} \mathbf{1}, B\right)$ and it is easy to see that $\operatorname{Hom}\left(\Sigma^{1} \mathbf{1}, B\right)$ is a homotopy comodule over $B$. We may now use that $\operatorname{hocoMod}_{l}(B)$ is a monoidal subcategory to conclude. 
Proposition 2.12 - We assume that the endofunctor $Q \otimes$ - of $\mathfrak{E}$ preserves weak equivalences for every cofibrant object $Q \in \mathfrak{E}$. Let $B \longrightarrow B^{\prime}$ be a morphism of homotopy bialgebras. There is an induced functor

$$
B^{\prime} \stackrel{\mathrm{L}}{\otimes}_{B}-: \operatorname{hocoMod}(B) \longrightarrow \operatorname{hocoMod}\left(B^{\prime}\right) .
$$

Moreover, if $B \longrightarrow B^{\prime}$ is a weak equivalence, this functor is an equivalence of categories.

Proof. We have a left Quillen functor

$$
B^{\prime} \otimes_{B}-: \operatorname{Mod}(B) \longrightarrow \operatorname{Mod}\left(B^{\prime}\right)
$$

which is a left Quillen equivalence if $B \longrightarrow B^{\prime}$ is a weak equivalence (see [15, Theorem 4.3]).

First, we check that $B^{\prime} \stackrel{\mathrm{L}}{\otimes}_{B}-$ takes a left homotopy comodule $M$ over $B$ to a left homotopy comodule over $B^{\prime}$. This amounts to check that, for $n \in \mathbb{N}$, the map

$$
B^{\prime n} \stackrel{\mathrm{L}}{\otimes}\left(B^{\prime 0} \stackrel{\mathrm{L}}{\otimes}_{B^{0}} M^{0}\right) \longrightarrow B^{\prime n} \stackrel{\mathrm{L}}{B_{B^{n}}} M^{n}
$$

is a weak equivalence. We fix a cofibrant replacement $U \longrightarrow M^{0}$. Arguing as in the proof of Proposition 2.11, we have weak equivalences

$$
B^{\prime n} \stackrel{\mathrm{L}}{\otimes}\left(B^{\prime 0} \stackrel{\mathrm{L}}{\otimes} B^{0} M^{0}\right) \longrightarrow B^{\prime n} \otimes U \quad \text { and } \quad B^{n} \otimes U \longrightarrow M^{n} .
$$

Thus, we are left to show that

$$
B^{\prime n} \otimes U \longrightarrow B^{\prime n} \stackrel{\mathrm{L}}{\otimes}_{B^{n}}\left(B^{n} \otimes U\right)
$$

is a weak equivalence, which is clear since $B^{n} \otimes U$ is a cofibrant object in $\operatorname{Mod}\left(B^{n}\right)$.

To finish the proof, it remains to show that the forgetful functor

$$
\mathbf{H o}\left(\operatorname{Mod}\left(B^{\prime}\right)\right) \longrightarrow \mathbf{H o}(\operatorname{Mod}(B))
$$

takes a left homotopy comodule over $B^{\prime}$ to a left homotopy comodule over $B$ (assuming that $B \longrightarrow B^{\prime}$ is a weak equivalence). This follows easily from the fact that

$$
B^{n} \stackrel{\mathrm{L}}{B_{B^{0}}} M^{0} \longrightarrow B^{\prime n} \stackrel{\mathrm{L}}{\otimes_{B^{\prime 0}}} M^{0}
$$

is a weak equivalence (use again [15, Theorem 4.3]).

\section{An ABSTRACT HOMOtOpiCAL SETting}

In this section, we will describe a situation which naturally gives rise to a homotopy Hopf algebra. Following the terminology in [1, §2.1.4], we will use "pseudo-monoidal functor" instead of "lax monoidal functor".

Setting 3.1 — Let $(\mathfrak{E}, \otimes, \mathbf{1})$ and $(\mathfrak{M}, \otimes, \mathbf{1})$ be two monoidal model categories related by a Quillen adjunction

$$
(c, \Gamma): \mathfrak{E} \rightleftarrows \mathfrak{M},
$$

where the left Quillen functor $c$ is monoidal. Let $\mathcal{S}$ be a pseudo-monoidal monad of $\mathfrak{M}$ (i.e., $\mathcal{S}$ is a pseudo-monoidal endofunctor of $\mathfrak{M}$ endowed with a unit $\mathrm{u}:$ id $\longrightarrow \mathcal{S}$ and a multiplication $\mathrm{m}: \mathcal{S} \circ \mathcal{S} \longrightarrow \mathcal{S}$ which are pseudo-monoidal natural transformations and which satisfy the usual rules of associativity and unitarity). We assume that the following conditions are satisfied. 
(a) The endofunctor $\mathcal{S}$ preserves fibrant objects and weak equivalences between fibrant objects. In particular, it admits a right derived functor

$$
\mathrm{RS}: \mathbf{H o}(\mathfrak{M}) \longrightarrow \mathbf{H o}(\mathfrak{M})
$$

which is pseudo-monoidal.

(b) The pseudo-monoidal functor

$$
\mathrm{R} \Gamma \circ \mathrm{RS}: \mathrm{Ho}(\mathfrak{M}) \longrightarrow \mathbf{H o}(\mathfrak{E})
$$

is monoidal. (In particular, the map $\mathbf{1} \longrightarrow \mathrm{R} \Gamma \circ \mathrm{RS}(\mathbf{1})$ is an isomorphism in $\operatorname{Ho}(\mathfrak{E})$.)

(c) For every objects $A, B \in \mathfrak{M}$, the natural maps

$$
A \stackrel{\mathrm{L}}{\otimes} \mathrm{RS}(B) \longrightarrow \mathrm{RS}(A \stackrel{\mathrm{L}}{\otimes} B) \quad \text { and } \quad \mathrm{RS}(A) \stackrel{\mathrm{L}}{\otimes} B \longrightarrow \mathrm{RS}(A \stackrel{\mathrm{L}}{\otimes} B)
$$

are isomorphisms in $\mathbf{H o}(\mathfrak{M})$.

Remark 3.2 — The first map in $(8)$ is given by the composition of

$$
A \stackrel{\mathrm{L}}{\otimes} \mathrm{RS}(B) \stackrel{\mathrm{u} \otimes \mathrm{id}}{\longrightarrow} \mathrm{RS}(A) \stackrel{\mathrm{L}}{\otimes} \mathrm{RS}(B) \stackrel{\mathrm{m}}{\longrightarrow} \mathrm{RS}(A \stackrel{\mathrm{L}}{\otimes} B),
$$

and the second map in (8) is defined similarly.

LEMMA 3.3 - We work under the hypothesis in Setting 3.1. For $A, B \in \mathfrak{M}$, and $m, n \in \mathbb{N}$, there is a canonical isomorphism in $\mathbf{H o}(\mathfrak{M})$ :

$$
\mathrm{RS}^{\circ m}(A) \stackrel{\mathrm{L}}{\otimes} \mathrm{R}^{\circ n}(B) \simeq \mathrm{R}^{\circ m+n}(A \stackrel{\mathrm{L}}{\otimes} B)
$$

given by the composition of

$$
\begin{aligned}
& \mathrm{RS}^{\circ m}(A) \stackrel{\mathrm{L}}{\otimes} \mathrm{RS}^{\circ n}(B) \\
& \left\lfloor\mathcal{S}^{\circ m}(\mathrm{u}) \otimes \mathrm{u}\left(\mathcal{S}^{\circ n}\right)\right. \\
& \mathrm{RS}^{\circ m} \circ \mathrm{RS}^{\circ n}(A) \stackrel{\mathrm{L}}{\otimes} \mathrm{RS}^{\circ m} \circ \mathrm{RS}^{\circ n}(B)=\mathrm{RS}^{\circ m+n}(A) \otimes \mathrm{L}^{\circ} \mathcal{L}^{m+n}(B) \\
& \downarrow \mathrm{m} \\
& \mathrm{RS}^{\circ m+n}(A \stackrel{\mathrm{L}}{\otimes} B) \text {. }
\end{aligned}
$$

Proof. First, we assume that $m=0$ and we argue by induction on $n$. When $n=0$, there is nothing to prove. When $n=1$, the claim is contained in condition (c) of Setting 3.1 . If $n \geqslant 2$, we use induction to get isomorphisms

$$
\begin{aligned}
A \otimes \mathrm{L}^{\circ}(B) & =A \stackrel{\mathrm{L}}{\otimes} \mathcal{R S}^{\circ n-1} \circ \mathrm{RS}(B) \\
& \simeq \mathrm{RS}^{\circ n-1}(A \otimes \mathrm{L} \mathcal{S}(B)) \\
& \simeq \mathrm{RS}^{\circ n-1} \circ \mathrm{RS}(A \otimes B) \\
& =\operatorname{RS}^{\circ n}(A \otimes B) .
\end{aligned}
$$

We leave it to the reader to check that the composition of these isomorphisms coincides with the composition in the statement. 
At this point, we know the lemma if $m=0$ or $n=0$ (by symmetry). Using these two cases, we may form the following chain of isomorphisms

$$
\begin{aligned}
& \mathrm{RS}^{\circ m}(A) \stackrel{\mathrm{L}}{\otimes} \mathrm{R}^{\circ n}(B) \simeq \mathrm{R}^{\circ m}\left(A \stackrel{\mathrm{L}}{\otimes} \mathrm{R}^{\circ n}(B)\right) \\
& \simeq \operatorname{RS}^{\circ m} \circ \mathrm{RS}^{\circ n}(A \stackrel{\mathrm{L}}{\otimes} B) \\
& =\mathrm{RS}^{\circ}+n(A \stackrel{\mathrm{L}}{\otimes} B) \text {. }
\end{aligned}
$$

Again, we leave it to the reader to check that the composition of these isomorphisms coincides with the composition in the statement.

Corollary 3.4 - We work under the hypothesis in Setting 3.1.

(a) For $n \in \mathbb{N}$, there is a canonical isomorphism of algebras in $\mathbf{H o}(\mathfrak{M})$ :

$$
\overbrace{\mathrm{RS}(\mathbf{1}) \stackrel{\mathrm{L}}{\otimes} \cdot \mathrm{L} \otimes \mathrm{RS}(\mathbf{1})}^{n \text { times }} \stackrel{\sim}{\longrightarrow} \mathrm{RS}^{\circ n}(\mathbf{1})
$$

which on the $m$-th factor, for $1 \leqslant m \leqslant n$, is given by the map

$$
\mathrm{RS}(\mathbf{1}) \stackrel{\mathrm{u}}{\longrightarrow} \mathrm{RS}^{\circ m-1} \circ \mathrm{RS} \circ \mathrm{RS}^{\circ n-m}(\mathbf{1})=\operatorname{RS}^{\circ n}(\mathbf{1}) .
$$

(b) For $M \in \mathfrak{M}$ and $n \in \mathbb{N}$, there is a canonical isomorphism in $\mathbf{H o}(\mathfrak{M})$ :

$$
\mathrm{RS}^{\circ n}(\mathbf{1}) \stackrel{\mathrm{L}}{\otimes} M \stackrel{\sim}{\longrightarrow} \mathrm{RS}^{\circ n}(M)
$$

which can be characterised as follows. It is a morphism of $\mathrm{RS}^{\circ n}(\mathbf{1})$-modules and its restriction to the second factor is given by $\mathrm{u}: M \longrightarrow \mathrm{RS}^{\circ}(M)$.

Proof. This is a direct consequence of Lemma 3.3 .

Proposition 3.5 - We work under the hypothesis in Setting 3.1. For $M \in \mathfrak{M}$, and $m, n \in \mathbb{N}$, there is a canonical isomorphism in $\mathbf{H o}(\mathfrak{E})$ :

$$
\mathrm{R} \Gamma \circ \mathrm{RS}^{\circ m+1}(\mathbf{1}) \stackrel{\mathrm{L}}{\otimes} \mathrm{R} \Gamma \circ \mathrm{RS}^{\circ n+1}(M) \stackrel{\sim}{\longrightarrow} \mathrm{R} \Gamma \circ \mathrm{RS}^{\circ m+n+1}(M)
$$

given by the composition of

$$
\begin{aligned}
& \mathrm{R} \Gamma \circ \mathrm{RS}^{\circ m+1}(\mathbf{1}) \stackrel{\mathrm{L}}{\otimes} \mathrm{R} \Gamma \circ \mathrm{RS}^{\circ n+1}(M) \\
& \left\lceil\left(\mathcal{S}^{\circ m+1}(\mathrm{u})\right) \otimes \Gamma\left(\mathrm{u}\left(\mathcal{S}^{\circ n+1}\right)\right)\right. \\
& \mathrm{R} \Gamma \circ \mathrm{RS}^{\circ m+1} \circ \mathrm{RS}^{\circ n}(\mathbf{1}) \stackrel{\mathrm{L}}{\otimes} \mathrm{R} \Gamma \circ \mathrm{RS}^{\circ m} \circ \mathrm{RS}^{\circ n+1}(M) \\
& \| \\
& \mathrm{R} \Gamma \circ \mathrm{RS}^{\circ m+n+1}(\mathbf{1}) \stackrel{\mathrm{L}}{\otimes} \mathrm{R} \Gamma \circ \mathrm{RS}^{\circ m+n+1}(M) \\
& \mathrm{m} \\
& \mathrm{R} \Gamma \circ \mathrm{RS}^{\circ m+n+1}(M) \text {. }
\end{aligned}
$$


Proof. By Corollary 3.4, we have isomorphisms

$$
\begin{aligned}
& \mathrm{R} \Gamma \circ \mathrm{RS}^{\circ m+1}(\mathbf{1}) \simeq \mathrm{R} \Gamma(\overbrace{\mathrm{RS}(\mathbf{1}) \stackrel{\mathrm{L}}{\otimes} \ldots \stackrel{\mathrm{L}}{\otimes} \mathrm{RS}(\mathbf{1})}^{m+1}), \\
& \mathrm{R} \Gamma \circ \mathrm{RS}^{\circ n+1}(M) \simeq \mathrm{R} \Gamma \overbrace{(\mathrm{RS}(\mathbf{1}) \otimes \ldots \otimes \mathrm{RS}(\mathbf{1})}^{n+1 \text { times }} \stackrel{\mathrm{L}}{\mathrm{L}} M) .
\end{aligned}
$$

Moreover, under these identifications, the composition in the statement can be rewritten as

$$
\begin{aligned}
& \mathrm{R} \Gamma\left(\mathrm{RS}(\mathbf{1})^{\mathrm{L} \otimes m} \stackrel{\mathrm{L}}{\otimes} \mathrm{RS}(\mathbf{1})\right) \stackrel{\mathrm{L}}{\otimes} \mathrm{R} \Gamma\left(\mathrm{RS}(\mathbf{1}) \stackrel{\mathrm{L}}{\otimes} \mathrm{RS}(\mathbf{1})^{\mathrm{L} \otimes n} \stackrel{\mathrm{L}}{\otimes} M\right) \\
& \mathrm{m} \\
& \mathrm{R} \Gamma(\mathrm{RS}(\mathbf{1})^{\mathrm{L} \otimes m} \stackrel{\mathrm{L}}{\otimes} \underbrace{\mathrm{RS}(\mathbf{1}) \stackrel{\mathrm{L}}{\otimes} \mathrm{RS}(\mathbf{1})}_{\mid m} \stackrel{\mathrm{L}}{\otimes} \mathrm{RS}(\mathbf{1})^{\mathrm{L} \otimes n} \stackrel{\mathrm{L}}{\otimes} M) \\
& \mathrm{R} \Gamma\left(\mathrm{R} \mathcal{S}(\mathbf{1})^{\mathrm{L} \otimes m} \stackrel{\mathrm{L}}{\otimes} \mathrm{RS}(\mathbf{1}) \stackrel{\mathrm{L}}{\otimes} \mathrm{RS}(\mathbf{1})^{\mathrm{L} \otimes n} \stackrel{\mathrm{L}}{\otimes} M\right) \\
& \mathrm{R} \Gamma\left(\mathrm{RS}(\mathbf{1})^{\mathrm{L} \otimes m+n+1} \stackrel{\mathrm{L}}{\otimes} M\right) .
\end{aligned}
$$

By applying the circular permutation $(1 \cdots m+1) \in \Sigma_{m+1}$ on $\mathrm{RS}(\mathbf{1})^{\mathrm{L} \otimes m+1}$, we may as well show that the composition of

$$
\begin{aligned}
& \mathrm{R} \Gamma\left(\mathrm{RS}(\mathbf{1}) \stackrel{\mathrm{L}}{\otimes} \mathrm{R} \mathcal{S}(\mathbf{1})^{\mathrm{L} \otimes m}\right) \stackrel{\mathrm{L}}{\otimes} \mathrm{R} \Gamma\left(\mathrm{RS}(\mathbf{1}) \stackrel{\mathrm{L}}{\otimes} \mathrm{R} \mathcal{S}(\mathbf{1})^{\mathrm{L} \otimes n} \stackrel{\mathrm{L}}{\otimes} M\right) \\
& \mathrm{m}
\end{aligned}
$$

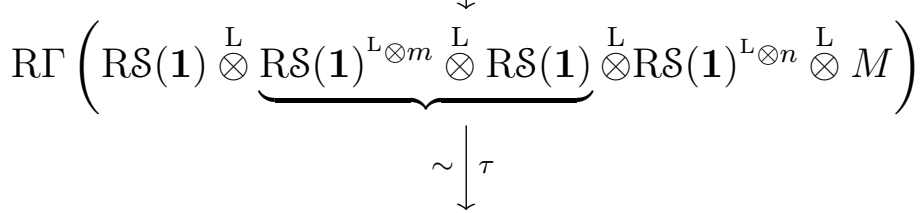

$$
\begin{aligned}
& \mathrm{R} \Gamma(\underbrace{\mathrm{R} \mathcal{S}(\mathbf{1}) \stackrel{\mathrm{L}}{\otimes} \mathrm{RS}(\mathbf{1})} \stackrel{\mathrm{L}}{\otimes} \mathrm{RS}(\mathbf{1})^{\mathrm{L} \otimes m} \stackrel{\mathrm{L}}{\otimes} \mathrm{RS}(\mathbf{1})^{\mathrm{L} \otimes n} \stackrel{\mathrm{L}}{\otimes} M) \\
& \downarrow \mathrm{m} \\
& \mathrm{R} \Gamma\left(\mathrm{RS}(\mathbf{1}) \stackrel{\mathrm{L}}{\otimes} \mathrm{RS}(\mathbf{1})^{\mathrm{L} \otimes m} \stackrel{\mathrm{L}}{\otimes} \mathrm{RS}(\mathbf{1})^{\mathrm{L} \otimes n} \stackrel{\mathrm{L}}{\otimes} M\right)
\end{aligned}
$$


is an isomorphism. Thus, it is more general to show that the composition of

$$
\begin{aligned}
& \mathrm{R} \Gamma(\mathrm{RS}(\mathbf{1}) \stackrel{\mathrm{L}}{\otimes} B) \stackrel{\mathrm{L}}{\otimes} \mathrm{R} \Gamma(\mathrm{RS}(\mathbf{1}) \stackrel{\mathrm{L}}{\otimes} C) \\
& \downarrow \mathrm{m} \\
& \mathrm{R} \Gamma(\mathrm{RS}(\mathbf{1}) \stackrel{\mathrm{L}}{\otimes} B \stackrel{\mathrm{L}}{\otimes} \mathrm{RS}(\mathbf{1}) \stackrel{\mathrm{L}}{\otimes} C) \stackrel{\tau}{\sim} \underset{\sim}{\sim} \mathrm{R} \Gamma(\mathrm{RS}(\mathbf{1}) \stackrel{\mathrm{L}}{\otimes} \mathrm{RS}(\mathbf{1}) \stackrel{\mathrm{L}}{\otimes} B \stackrel{\mathrm{L}}{\otimes} C) \\
& \downarrow \mathrm{m} \\
& \mathrm{R} \Gamma(\mathrm{RS}(\mathbf{1}) \stackrel{\mathrm{L}}{\otimes} B \stackrel{\mathrm{L}}{\otimes} C)
\end{aligned}
$$

is an isomorphism for all $B, C \in \mathfrak{M}$. Using the isomorphism $\mathrm{RS}(\mathbf{1}) \otimes-\simeq \mathrm{RS}(-)$, we may identify the composition above with the composition of

$$
\mathrm{R} \Gamma(\mathrm{RS}(B)) \stackrel{\mathrm{L}}{\otimes} \mathrm{R} \Gamma(\mathrm{RS}(C)) \stackrel{\mathrm{m}}{\longrightarrow} \mathrm{R} \Gamma(\mathrm{RS}(B) \stackrel{\mathrm{L}}{\otimes} \mathrm{RS}(C)) \stackrel{\mathrm{m}}{\longrightarrow} \mathrm{R} \Gamma(\mathrm{RS}(B \stackrel{\mathrm{L}}{\otimes} C))
$$

which is an isomorphism by condition (b) of Setting 3.1 .

CoRollary 3.6 - We work under the hypothesis in Setting 3.1. For $n \in \mathbb{N}$, there is a canonical isomorphism of algebras in $\mathbf{H o}(\mathfrak{E})$ :

$$
\overbrace{\mathrm{R} \Gamma \circ \mathrm{RS}^{\circ 2}(\mathbf{1}) \otimes \cdots \otimes \mathrm{L} \Gamma \circ \mathrm{RS}^{\circ 2}(\mathbf{1})}^{\mathrm{L}^{\text {times }}} \stackrel{\mathrm{L}}{\sim} \mathrm{R} \Gamma \circ \mathrm{RS}^{\circ n+1}(\mathbf{1})
$$

which on the $m$-th factor, for $1 \leqslant m \leqslant n$, is given by the map

$$
\mathrm{R} \Gamma \circ \mathrm{RS}^{\circ 2}(\mathbf{1}) \stackrel{\mathrm{u}}{\longrightarrow} \mathrm{R} \Gamma \circ\left(\mathrm{RS}^{\circ m-1} \circ \mathrm{RS}^{\circ 2} \circ \mathrm{RS}^{\circ n-m}(\mathbf{1})\right)=\mathrm{R} \Gamma \circ \mathrm{RS}^{\circ n+1}(\mathbf{1}) .
$$

Proof. This is a direct consequence of Proposition 3.5 .

Corollary $3.7-$ We work under the hypothesis in Setting 3.1. For $M \in \mathfrak{M}$ and $n \in \mathbb{N}$, there is a canonical isomorphism in $\mathbf{H o}(\mathfrak{E})$ :

$$
\mathrm{R} \Gamma \circ \mathrm{RS}^{\circ n+1}(\mathbf{1}) \stackrel{\mathrm{L}}{\otimes} \mathrm{R} \Gamma \circ \mathrm{RS}(M) \stackrel{\sim}{\longrightarrow} \mathrm{R} \Gamma \circ \mathrm{RS}^{\circ n+1}(M)
$$

which can be characterised as follows. It is a morphism of $\mathrm{R} \Gamma \circ \mathrm{RS}^{\circ n+1}(\mathbf{1})$-modules and its restriction to the second factor is given by

$$
\mathrm{u}: \mathrm{R} \Gamma \circ \mathrm{RS}(M) \stackrel{\mathrm{u}}{\longrightarrow} \mathrm{R} \Gamma \circ \mathrm{RS}^{\circ n} \circ \mathrm{RS}(M)=\mathrm{R} \Gamma \circ \mathrm{RS}^{\circ n+1}(M) .
$$

Proof. This is a particular case of Proposition 3.5 .

We will also need the following variant of (a special case of) Proposition 3.5.

LEмма 3.8 - We work under the hypothesis in Setting 3.1. There are two

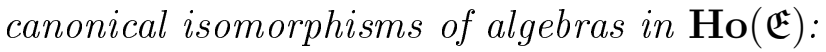

$$
\delta_{1} \cdot \delta_{0} \quad \text { and } \quad \delta_{2} \cdot \delta_{1}: \mathrm{R} \Gamma \circ \mathrm{RS}^{\circ 2}(\mathbf{1}) \stackrel{\mathrm{L}}{\otimes} \mathrm{R} \Gamma \circ \mathrm{RS}^{\circ 2}(\mathbf{1}) \stackrel{\sim}{\longrightarrow} \mathrm{R} \Gamma \circ \mathrm{RS}^{\circ 3}(\mathbf{1})
$$

where:

$$
\delta_{0}: \mathrm{R} \Gamma(\mathrm{RS} \circ \mathrm{RS}(\mathbf{1}))=\mathrm{R} \Gamma(\mathrm{id} \circ \mathrm{RS} \circ \mathrm{RS}(\mathbf{1})) \stackrel{\mathrm{u}}{\longrightarrow} \mathrm{R} \Gamma(\mathrm{RS} \circ \mathrm{RS} \circ \mathrm{RS}(\mathbf{1})),
$$




$$
\begin{aligned}
& \delta_{1}: \mathrm{R} \Gamma(\operatorname{RS} \circ \operatorname{RS}(\mathbf{1}))=\mathrm{R} \Gamma(\operatorname{RS} \circ \mathrm{id} \circ \operatorname{RS}(\mathbf{1})) \stackrel{\mathrm{u}}{\longrightarrow} \mathrm{R} \Gamma(\operatorname{RS} \circ \operatorname{RS} \circ \operatorname{RS}(\mathbf{1})) \\
& \delta_{2}: \operatorname{R} \Gamma(\operatorname{RS} \circ \operatorname{RS}(\mathbf{1}))=\operatorname{R} \Gamma(\operatorname{RS} \circ \operatorname{RS} \circ \mathrm{id}(\mathbf{1})) \stackrel{\mathrm{u}}{\longrightarrow} \mathrm{R} \Gamma(\operatorname{RS} \circ \operatorname{RS} \circ \operatorname{RS}(\mathbf{1}))
\end{aligned}
$$

Proof. The argument used in the proof of Proposition 3.5 can be easily adapted to prove the lemma. We leave the details to the reader.

Construction 3.9 - We work under the hypothesis in Setting 3.1. The monad $\mathcal{S}$ gives rise to a cosimplicial pseudo-monoidal endofunctor $\widehat{\mathcal{S}}$ of $\mathfrak{M}$ as follows.

(1) For $n \in \mathbb{N}$, we set

$$
\widehat{\mathcal{S}}^{n}=\mathcal{S}^{\circ n+1} .
$$

(2) For $0 \leqslant i \leqslant n$, the natural transformation $\sigma_{i}: \widehat{\mathcal{S}}^{n+1} \longrightarrow \widehat{\mathcal{S}}^{n}$ is given by

$$
\mathcal{S}^{\circ n+2}=\mathcal{S}^{\circ i} \circ \mathcal{S}^{\circ 2} \circ \mathcal{S}^{\circ n-i} \stackrel{\text { idomoid }}{\longrightarrow} \mathcal{S}^{\circ i} \circ \mathcal{S} \circ \mathcal{S}^{\circ n-i}=\mathcal{S}^{\circ n+1} .
$$

(3) For $0 \leqslant i \leqslant n+1$, the natural tranformation $\delta_{i}: \widehat{\mathcal{S}}^{n} \longrightarrow \widehat{\mathcal{S}}^{n+1}$ is given by

$$
\mathcal{S}^{\circ n+1}=\mathcal{S}^{\circ i} \circ \text { id } \circ \mathcal{S}^{\circ n+1-i} \stackrel{\text { idouoid }}{\longrightarrow} \mathcal{S}^{\circ i} \circ \mathcal{S} \circ \mathcal{S}^{\circ n+1-i}=\mathcal{S}^{\circ n+2} .
$$

Given an algebra $A$ in $\mathfrak{M}, \widehat{\mathcal{S}}(A)$ is a cosimplicial algebra in $\mathfrak{M}$.

THEOREM 3.10 - We work under the hypothesis in Setting 3.1. We fix a commutative algebra $A$ in $\mathfrak{M}$ which is fibrant (as an object of $\mathfrak{M}$ ) and such that the map u : $1 \longrightarrow A$ is a weak equivalence..$^{5}$

(a) The cosimplicial algebra $\Gamma \circ \widehat{\mathcal{S}}(A)$ is a homotopy Hopf algebra in $\mathfrak{E}$.

(b) Let $M$ be an A-module which is fibrant as an object of $\mathfrak{M}$. Then $\Gamma \circ \widehat{\mathcal{S}}(M)$ is a homotopy comodule over $\Gamma \circ \widehat{\mathcal{S}}(A)$.

Proof. As $A$ is a commutative algebra in $\mathfrak{M}, \Gamma \circ \widehat{\mathcal{S}}(A)$ is a cosimplicial commutative algebra in $\mathfrak{E}$. Furthermore, by the choice of $A$, we have isomorphisms in $\mathbf{H o}(\mathfrak{E})$ :

$$
\Gamma \circ \mathcal{S}^{\circ n+1}(A) \simeq \mathrm{R} \Gamma \circ \mathrm{RS}^{\circ n+1}(\mathbf{1}) .
$$

Thus, Corollary 3.6 implies that $\Gamma \circ \widehat{\mathcal{S}}(A)$ is a homotopy bialgebra and Lemma 3.8 insures that this homotopy bialgebra is in fact a homotopy Hopf algebra. This proves part (a) of the statement.

We now prove (b). As $M$ is assumed to be fibrant, we have isomorphisms in $\operatorname{Ho}(\mathfrak{E})$ :

$$
\Gamma \circ \mathcal{S}^{\circ n+1}(M) \simeq \mathrm{R} \Gamma \circ \mathrm{RS}^{\circ n+1}(M) .
$$

Thus, Corollary 3.7 implies that the $\Gamma \circ \widehat{\mathcal{S}}(A)$-module $\Gamma \circ \widehat{\mathcal{S}}(M)$ is a left homotopy comodule. (As $\Gamma \circ \widehat{\mathcal{S}}(A)$ is a homotopy Hopf algebra, we may speak of homotopy comodule thanks to Proposition 2.6.)

THEOREM 3.11 - We work under the hypothesis in Setting 3.1. We also assume the following technical conditions.

- The model categories $\mathfrak{M}$ and $\mathfrak{E}$ are presentable by cofibrations as in [2, Définition 4.2.39]. (Another possibility is to assume that $\mathfrak{E}$ is cellular as in [8, Definition 12.1.1].)

\footnotetext{
${ }^{5}$ It is not always possible to find such a commutative algebra; see for example [15, Remark 4.5].
} 
- The monoidal model categories $\mathfrak{M}$ and $\mathfrak{E}$ satisfy the monoid axiom as in [15, Definition 3.3].

- The endofunctor $M \otimes-($ resp. $Q \otimes-)$ of $\mathfrak{M}$ (resp. E) preserves weak equivalences for every cofibrant object $M \in \mathfrak{M}$ (resp. $Q \in \mathfrak{E})$.

Fix a commutative algebra $A$ in $\mathfrak{M}$ which is fibrant (as an object of $\mathfrak{M}$ ) and such that the map $\mathrm{u}: \mathbf{1} \longrightarrow A$ is a weak equivalence. Then, there exists a monoidal functor

$$
\mathrm{R} \Gamma \circ \mathrm{R} \widehat{\mathcal{S}}(A \stackrel{\mathrm{L}}{\otimes}-): \mathbf{H o}(\mathfrak{M}) \longrightarrow \operatorname{hocoMod}(\Gamma \circ \widehat{\mathcal{S}}(A)) .
$$

Assuming that $\mathfrak{M}$ and $\mathfrak{E}$ are stable, the functor $(9)$ is triangulated.

Proof. By [15, Theorem 4.3], we have a left Quillen equivalence $\mathfrak{M} \longrightarrow \operatorname{Mod}(A)$ yielding an equivalence of categories

$$
A \stackrel{\mathrm{L}}{\otimes}-: \mathbf{H o}(\mathfrak{M}) \stackrel{\sim}{\longrightarrow} \mathbf{H o}(\operatorname{Mod}(A)) .
$$

On the other hand, we have a pseudo-monoidal functor

$$
\Gamma \circ \widehat{\mathcal{S}}: \operatorname{Mod}(A) \longrightarrow \operatorname{Mod}(\widehat{\mathcal{S}}(A))
$$

which preserves weak equivalences between fibrant $A$-modules. Thus, it admits a right derived functor

$$
\mathrm{R} \Gamma \circ \mathrm{R} \widehat{\mathcal{S}}: \mathbf{H o}(\operatorname{Mod}(A)) \longrightarrow \mathbf{H o}(\operatorname{Mod}(\widehat{\mathcal{S}}(A))) .
$$

By Theorem 3.10, the image of this functor is contained in $\operatorname{hocoMod}(\widehat{\mathcal{S}}(A))$. This gives the functor (9) of the statement.

Using Proposition 2.11, it follows that the functor $(9)$ is pseudo-monoidal and triangulated under the appropriate technical assumptions. Thus, it remains to see that $(9)$ is monoidal. This follows from Lemma 2.5 and the fact that

$$
\mathrm{R} \Gamma \circ \mathrm{R} \widehat{\mathcal{S}}^{0}=\mathrm{R} \Gamma \circ \mathrm{RS}: \mathbf{H o}(\mathfrak{M}) \longrightarrow \mathbf{H o}(\mathfrak{E})
$$

is a monoidal functor (by condition (b) in Setting 3.1).

We end this section with the following compatibility result with the weak Tannakian formalism of [5, §1].

THEOREM 3.12 - We work under the hypothesis in Setting 3.1 and we assume the following two further properties.

(i) The functor $f=\mathrm{R} \Gamma \circ \mathrm{RS}$ admits a right adjoint $g: \mathbf{H o}(\mathfrak{E}) \longrightarrow \mathbf{H o}(\mathfrak{M})$. Moreover, the natural transformation $\mathrm{RS} \longrightarrow g \circ f$, which is adjoint to the composition of

$$
f \circ \mathrm{RS}=\mathrm{R} \Gamma \circ \mathrm{RS}^{\circ 2} \stackrel{\mathrm{m}}{\longrightarrow} \mathrm{R} \Gamma \circ \mathrm{RS}=f,
$$

is an isomorphism.

(ii) The composition of

$$
\mathrm{id} \stackrel{\eta}{\longrightarrow} \mathrm{R} \Gamma \circ \mathrm{L} c \stackrel{\mathrm{u}}{\longrightarrow} \mathrm{R} \Gamma \circ \mathrm{RS} \circ \mathrm{L} c=f \circ \mathrm{L} c
$$

is an isomorphism of endofunctors of $\mathbf{H o}(\mathfrak{E})$. In particular, Lc is a monoidal section to $f$.

Then, the functor $f$, with its monoidal section Lc, satisfies [5, Hypothèse 1.40]. Furthermore, the following two conclusions hold. 
(a) The Hopf algebra $\mathrm{H}=f \circ g(\mathbf{1})$ of $\mathbf{H o}(\mathfrak{E})$, given by [5., Théorème 1.21] (and [5, Théorème 1.45]), is canonically isomorphic to the Hopf algebra $\mathrm{R} \Gamma \circ \mathrm{RS}^{\circ 2}(\mathbf{1})$ that one gets from Theorem 3.10(a) via Proposition 1.4.

(b) For $M \in \mathbf{H o}(\mathfrak{M})$, the left coaction of $\mathrm{H}$ on $f(M)$, given by [5, Proposition $1.28(\mathrm{a})]$, coincides with the left coaction of $\mathrm{R} \Gamma \circ \mathrm{RS}^{\circ 2}(\mathbf{1})$ on $\mathrm{R} \Gamma \circ \mathrm{RS}(M)$ that one gets from Theorem 3.11 (see also Theorem 3.10(b)) via Proposition 1.7 (modulo the Hopf algebras isomorphism of (a)).

The rest of this section is devoted to the proof of this theorem. Thus, until the end of this section, we work under the assumptions of the statement of Theorem 3.12 ,

Remark 3.13 - From the assumption (ii) of the statement of Theorem 3.12 , there is a natural isomorphism of endofunctors of $\mathbf{H o}(\mathfrak{E})$ :

$$
\text { id } \stackrel{\sim}{\longrightarrow} f \circ \mathrm{L} c .
$$

Passing to right adjoints, one also gets a natural isomorphism of endofunctors:

$$
\mathrm{R} \Gamma \circ g \stackrel{\sim}{\longrightarrow} \mathrm{id} .
$$

Notation 3.14 - We denote by $\alpha: f \circ R \mathcal{R} \longrightarrow f$ the composition of

$$
f \circ \mathrm{RS}=\mathrm{R} \Gamma \circ \mathrm{RS} \circ \mathrm{RS} \stackrel{\mathrm{m}}{\longrightarrow} \mathrm{R} \Gamma \circ \mathrm{RS}=f
$$

and we denote by $\theta: \mathrm{RS} \longrightarrow g \circ f$ the natural transformation obtained from $\alpha$ by adjunction. By the assumption (i) of the statement of Theorem 3.12, $\theta$ is an isomorphism.

LEMMA 3.15 - The invertible natural transformation $\theta: \mathrm{RS} \stackrel{\sim}{\longrightarrow} g \circ f$ is an isomorphism of pseudo-monoidal monads.

Proof. It is easy to see that $\theta$ is a morphism of pseudo-monoidal endofunctors. We will only check that $\theta$ is a morphism of monads. By adjunction, the composition of

$$
\mathrm{id} \stackrel{\mathrm{u}}{\longrightarrow} \mathrm{RS} \stackrel{\theta}{\longrightarrow} g \circ f
$$

corresponds to the composition of

$$
f \stackrel{\mathrm{u}}{\longrightarrow} f \circ \mathrm{RS}=\mathrm{R} \Gamma \circ \mathrm{RS} \circ \mathrm{RS} \stackrel{\mathrm{m}}{\longrightarrow} \mathrm{R} \Gamma \circ \mathrm{RS}=f
$$

which is clearly equal to the identity of $f$. This shows that $\theta$ is compatible with the unit maps.

It remains to prove that $\theta$ is compatible with multiplication, i.e., that the square

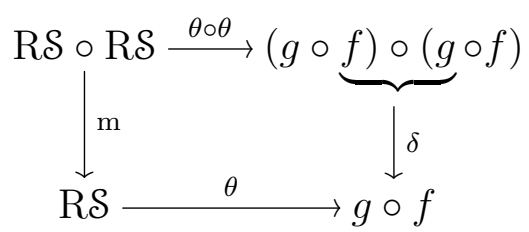

is commutative. To do this, we expand the square into the following diagram

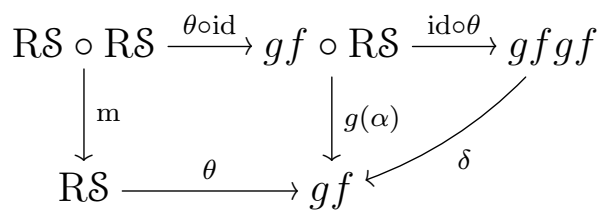

and check that each subdiagram commutes. 
The commutativity of the triangle in $(12)$ follows the commutativity of the diagram

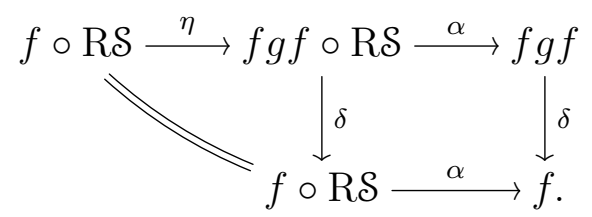

The square in 12 can be expanded as follows

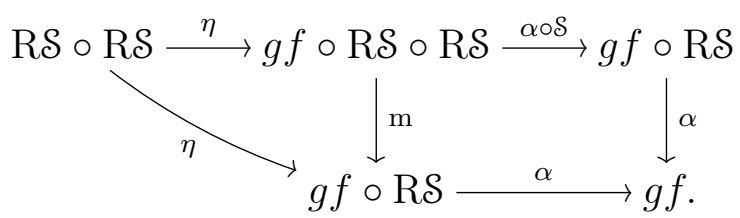

The left triangle commutes for obvious reasons and the commutativity of the square follows from the associativity of the multiplication of $\mathcal{S}$.

We can now prove the following bit of the statement of Theorem 3.12 .

Corollary 3.16 - The monoidal functor $f$ satisfies [5, Hypothèse 1.40].

Proof. The functor $\mathrm{L} c$ admits a right adjoint, namely $\mathrm{R} \Gamma$. It remains to show that the natural morphism

$$
g\left(A^{\prime}\right) \otimes B \longrightarrow g\left(A^{\prime} \otimes f(B)\right)
$$

is an isomorphism for $A^{\prime} \in \mathbf{H o}(\mathfrak{E})$ and $B \in \mathbf{H o}(\mathfrak{M})$. Since the functor $f$ admits a section, it is surjective on objects, and thus we may assume that $A^{\prime}=f(A)$ for some $A \in \mathbf{H o}(\mathfrak{M})$. Moreover, the composition of

$$
g f(A) \otimes B \longrightarrow g(f(A) \otimes f(B)) \simeq g f(A \otimes B)
$$

coincides with the composition of

$$
g f(A) \otimes B \stackrel{\mathrm{id} \otimes \eta}{\longrightarrow} g f(A) \otimes g f(B) \stackrel{\mathrm{m}}{\longrightarrow} g f(A \otimes B) .
$$

Using the isomorphism of pseudo-monoidal monads $\mathrm{RS} \simeq g \circ f$ of Lemma 3.15] the result follows from the assumption (c) in Setting 3.1 .

Notation 3.17 - For $n \in \mathbb{N} \sqcup\{-1\}$, we denote by

$$
\rho_{n}: \mathrm{R} \Gamma \circ \mathrm{R}^{1+n} \stackrel{\sim}{\longrightarrow} f \circ{\widehat{(g \circ f)^{n}}}^{n}
$$

the composition of

$$
\mathrm{R} \Gamma \circ \mathrm{RS}^{\circ 2+n} \stackrel{\theta^{2+n}}{\sim} \mathrm{R} \Gamma \circ g \circ f \circ(g \circ f)^{\circ 1+n} \stackrel{\sim}{\longrightarrow} \circ(f \circ g)^{\circ 1+n},
$$

where the last isomorphism is induced from $(11)$. (By convention, $\overline{(g \circ f)}^{-1}$ is the identity functor.)

LEMMA 3.18 - The natural transformations $\rho_{n}$, for $n \in \mathbb{N} \sqcup\{-1\}$, define an isomorphism of coaugmented cosimplicial functors

$$
\rho: \mathrm{R} \Gamma \circ \mathrm{R}^{1+\bullet} \stackrel{\sim}{\longrightarrow} f \circ \overline{(g \circ f)} \bullet
$$

\footnotetext{
${ }^{6}$ In fact, we only need to know that $\mathrm{R} \mathcal{S} \simeq g \circ f$ is an isomorphism of pseudo-monoidal functors which is compatible with the unit maps.
} 
In particular, the following squares

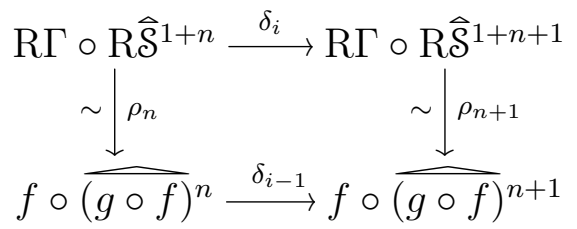

commute for all $1 \leqslant i \leqslant n+2$.

Proof. This is a direct consequence of Lemma 3.15 and the definition of the natural transformations $\rho_{n}$.

Notation 3.19 — We denote by $\gamma:$ id $\longrightarrow f \circ g$ the natural transformation given by the composition of

$$
\mathrm{id} \stackrel{\sqrt{10}}{\sim} f \circ \mathrm{L} c \stackrel{\eta}{\longrightarrow} f \circ g \circ f \circ \mathrm{L} c \stackrel{\left[10^{-1}\right.}{\sim} f \circ g .
$$

Equivalently, $\gamma$ is also the composition of

$$
\mathrm{id} \stackrel{\mathbb{1 1}^{-1}}{\sim} \mathrm{R} \Gamma \circ g \stackrel{\eta}{\longrightarrow} \mathrm{R} \Gamma \circ g \circ f \circ g \stackrel{11}{\sim} f \circ g .
$$

(We leave the verification of this claim to the reader.)

Remark 3.20 - Modulo the isomorphism $1 \simeq f(\mathbf{1})$, the two maps

$$
\gamma(\mathbf{1}): \mathbf{1} \longrightarrow f g(\mathbf{1}) \quad \text { and } \quad f(\eta): f(\mathbf{1}) \longrightarrow f g f(\mathbf{1})
$$

coincide since both of them are unit maps for the algebra $f g(\mathbf{1})$.

LEMMA 3.21 - For $n \in \mathbb{N} \sqcup\{-1\}$, the composition of

$$
\mathrm{R} \Gamma \circ \mathrm{R} \widehat{\mathcal{S}}^{n+1} \stackrel{\delta_{0}}{\longrightarrow} \mathrm{R} \Gamma \circ \mathrm{R} \widehat{\mathcal{S}}^{1+n+1} \stackrel{\rho_{n+1}}{\sim} f \circ \widehat{(g \circ f}^{n+1}
$$

is equal to the composition of

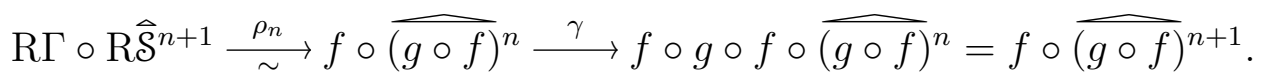

Proof. It is clearly enough to treat the case $n=-1$. Thus, we need to analyse the composition of

$$
\mathrm{R} \Gamma \circ \mathrm{RS}=\mathrm{R} \Gamma \circ \mathrm{id} \circ \mathrm{RS} \stackrel{\mathrm{u}}{\longrightarrow} \mathrm{R} \Gamma \circ \mathrm{RS} \circ \mathrm{RS} \stackrel{\theta^{2}}{\longrightarrow} \mathrm{R} \Gamma \circ g f g f \stackrel{\sim}{\longrightarrow} f g f .
$$

By Lemma 3.15, this composition is equal to

$$
\mathrm{R} \Gamma \circ \mathrm{RS} \stackrel{\theta}{\longrightarrow} \overbrace{\mathrm{R} \Gamma \circ g f=\mathrm{R} \Gamma \circ \mathrm{id} \circ g f \stackrel{\eta}{\longrightarrow} \mathrm{R} \Gamma \circ g f g f \stackrel{\sim}{\longrightarrow} f g f}^{\longrightarrow} .
$$

Now, recall that $\gamma:$ id $\longrightarrow f g$ is the composition of

$$
\mathrm{id} \simeq \mathrm{R} \Gamma \circ g \stackrel{\eta}{\longrightarrow} \mathrm{R} \Gamma \circ g f g \simeq f g .
$$

This shows that the composition of the embraced portion in 13 is equal to the composition of

$$
\mathrm{R} \Gamma \circ g f \simeq f \stackrel{\gamma}{\longrightarrow} f g f .
$$

This finishes the proof of the lemma. 
Theorem 3.12 follows from Propositions 3.22 and 3.23 below.

Proposition 3.22 - The isomorphism

$$
\rho=\rho_{0}(\mathbf{1}): \mathrm{R} \Gamma \circ \mathrm{RS}^{\circ 2}(\mathbf{1}) \stackrel{\sim}{\longrightarrow} f g(\mathbf{1})=\mathrm{H}
$$

is an isomorphism of Hopf algebras. (See the statement of Theorem 3.12 for the description of these Hopf algebras.)

Proof. It is easy to see that $\rho$ is a morphism of algebras. We will only show that $\rho$ is compatible with comultiplication.

The comultiplication on $\mathrm{R} \Gamma \circ \mathrm{RS}^{\circ 2}(\mathbf{1})$ is given by the composition of

$$
\mathrm{R} \Gamma \circ \mathrm{RS}^{\circ 2}(\mathbf{1}) \stackrel{\delta_{1}}{\longrightarrow} \mathrm{R} \Gamma \circ \mathrm{RS}^{\circ 3}(\mathbf{1}) \stackrel{\delta_{2} \cdot \delta_{0}}{\sim} \mathrm{R} \Gamma \circ \mathrm{RS}^{2}(\mathbf{1}) \otimes \mathrm{R} \Gamma \circ \mathrm{RS}^{2}(\mathbf{1})
$$

(see the proof of Proposition 1.4). By Lemmas 3.18 and 3.21, we have a commutative diagram

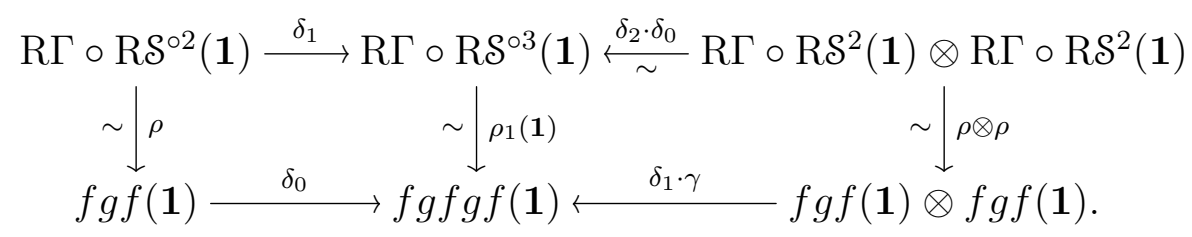

Identifying $f(\mathbf{1})$ with $\mathbf{1}, \delta_{0}$ is simply $\eta: f g(\mathbf{1}) \longrightarrow f g f g(\mathbf{1})$. Thus, comparing with the comultiplication as defined in [5, Théorème 1.21], we need to identify the map

$$
f g f(\eta) \cdot \gamma(f g f(\mathbf{1})): f g f(\mathbf{1}) \otimes f g f(\mathbf{1}) \longrightarrow f g f g f(\mathbf{1})
$$

with the composition of

$$
\begin{aligned}
f g(\mathbf{1}) \otimes f g(\mathbf{1}) & \simeq f g(\mathbf{1}) \otimes f \circ \mathrm{Lc} \circ f g(\mathbf{1}) \\
& \simeq f(g(\mathbf{1}) \otimes \mathrm{L} c \circ f g(\mathbf{1})) \\
& \stackrel{c_{d}}{\longrightarrow} f g(\mathbf{1} \otimes f \circ \mathrm{L} c \circ f g(\mathbf{1})) \\
& \simeq f g f g(\mathbf{1}) .
\end{aligned}
$$

As both maps are morphisms of algebras, it is enough to check that they coincide on each of the two $f g(\mathbf{1})$ factors.

On the left $f g(\mathbf{1})$ factor, (14) is simply

$$
f g(\mathrm{u}): f g(\mathbf{1}) \longrightarrow f g f g(\mathbf{1})
$$

where $\mathrm{u}: \mathbf{1} \longrightarrow f g(\mathbf{1})$ is the unit of algebra $f g(\mathbf{1})$ which is also equal to $f(\eta)$ : $f(\mathbf{1}) \longrightarrow f g f(\mathbf{1})$ modulo the identification $f(\mathbf{1}) \simeq \mathbf{1}$. This proves what we need for the left $f g(\mathbf{1})$ factor.

On the right $f g(\mathbf{1})$ factor, (14) is given by the composition of

$$
f g(\mathbf{1}) \simeq f \circ \mathrm{L} c \circ f g(\mathbf{1}) \stackrel{\eta}{\longrightarrow} f g f \circ \operatorname{Lc} \circ f g(\mathbf{1}) \simeq f g f g(\mathbf{1})
$$

which is precisely $\gamma(f g(\mathbf{1}))$ as needed. This finishes the proof of the proposition.

Proposition 3.23 - Let $M \in \mathbf{H o}(\mathfrak{M})$. The identification

$$
\mathrm{R} \Gamma \circ \mathrm{RS}(M)=f(M)
$$


is compatible with the left coaction of $\mathrm{R} \Gamma \circ \mathrm{RS}^{\circ 2}(\mathbf{1})$ on $\mathrm{R} \Gamma \circ \mathrm{RS}(M)$ and the left coaction of $f g(\mathbf{1})$ on $f(M)$. (See the statement of Theorem 3.12 for the description of these coactions.)

Proof. The coaction of $\mathrm{R} \Gamma \circ \mathrm{RS}^{\circ 2}(\mathbf{1})$ on $\mathrm{R} \Gamma \circ \mathrm{RS}(M)$ is given by the composition of

$$
\mathrm{R} \Gamma \circ \mathcal{S}(M) \stackrel{\delta_{1}}{\longrightarrow} \mathrm{R} \Gamma \circ \mathcal{S}^{\circ 2}(M) \stackrel{\delta_{0}}{\sim} \mathrm{R} \Gamma \circ \mathcal{S}^{\circ 2}(\mathbf{1}) \otimes \mathrm{R} \Gamma \circ \mathcal{S}(M)
$$

(see the proof of Proposition 1.7 and remark that $\iota_{0}=\delta_{1}$ and $\iota_{1}=\delta_{0}$ ). By Lemmas 3.18 and 3.21 , we have a commutative diagram

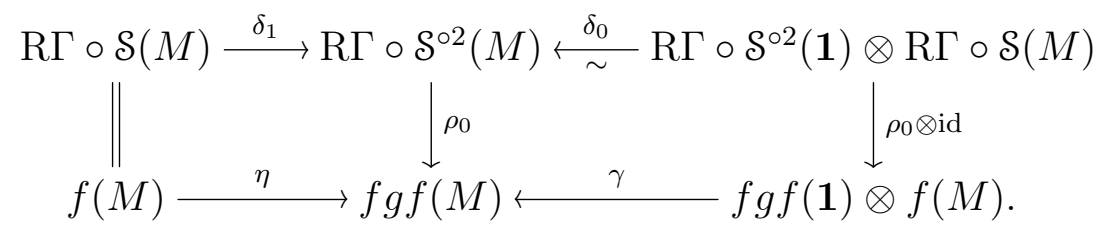

Thus, comparing with the coaction as defined in [5, Proposition 1.28(a)], we need to identify the map

$$
\gamma: f g f(\mathbf{1}) \otimes f(M) \longrightarrow f g f(M)
$$

(and more precisely, the $f g f(\mathbf{1})$-linear extension of $\gamma: f(M) \longrightarrow f g f(M)$ ) with the composition of

$$
\begin{aligned}
f g(\mathbf{1}) \otimes f(M) & \simeq f g(\mathbf{1}) \otimes f \circ \operatorname{Lc} \circ f(M) \\
& \simeq f(g(\mathbf{1}) \otimes \operatorname{L} c \circ f(M)) \\
& \stackrel{c_{d}}{\longrightarrow} f g(\mathbf{1} \otimes f \circ \operatorname{Lc} \circ f(M)) \\
& \simeq f g f(M) .
\end{aligned}
$$

As both maps are morphisms of $f g(\mathbf{1})$-modules, it is enough to show that the restriction of 15 to $f(M)$ is equal to $\gamma: f(M) \longrightarrow f g f(M)$ which is clear.

\section{Commutative spectra}

Let $(\mathfrak{M}, \otimes, \mathbf{1})$ be a monoidal model category and let $T \in \mathfrak{M}$ be a cofibrant object. By [10], under some mild technical assumptions, we may form two model categories $\mathbf{S p t}_{T}(\mathfrak{M})$ and $\mathbf{S p t}_{T}^{\Sigma}(\mathfrak{M})$, whose objects are called $T$-spectra and symmetric $T$-spectra respectively. These two model categories turn out to be Quillen equivalent in some favourable situations (see for example [10, Corollary 10.4] or [2, Théorème 4.3.79]). Furthermore, the second category has a monoidal structure (which is compatible with the model structure under some further technical assumptions) whereas the first one does not. For more details, we refer the reader to [10] and [2, §4.3].

Assuming that $\mathfrak{M}$ is $\mathbb{Q}$-linear, we introduce in this section a third model category $\operatorname{Spt}_{T}^{\sharp}(\mathfrak{M})$ whose objects will be called commutative $T$-spectra (for lack of a better name). In some sense, $\mathbf{S p t}_{T}^{\sharp}(\mathfrak{M})$ is as simple as $\mathbf{S p t}_{T}(\mathfrak{M})$ and, at the same time, retains the good formal properties from $\operatorname{Spt}_{T}^{\Sigma}(\mathfrak{M})$. In particular, $\operatorname{Spt}_{T}^{\sharp}(\mathfrak{M})$ is a monoidal category. Also, in some favourable situations, we will see that $\operatorname{Spt}_{T}^{\sharp}(\mathfrak{M})$ is Quillen equivalent to both $\mathbf{S p t}_{T}(\mathfrak{M})$ and $\operatorname{Spt}_{T}^{\Sigma}(\mathfrak{M})$.

If not otherwise stated, in this section $(\mathfrak{M}, \otimes, \mathbf{1})$ will be a monoidal model category with cofibrant unit and satisfying the following assumptions. 


\section{Hypothesis 4.1}

- The model category $\mathfrak{M}$ is presentable by cofibrations as in 2, Définition 4.2.39]. (Another possibility is to assume that $\mathfrak{M}$ is cellular as in [8, Definition 12.1.1].)

- Coproducts and filtered colimits in $\mathfrak{M}$ preserve weak equivalences.

- The category $\mathfrak{M}$ is additive and $\mathbb{Q}$-linear.

Remark 4.2 - Given a finite group $G$ acting on an object $M \in \mathfrak{M}$, the assumption that $\mathfrak{M}$ is additive and $\mathbb{Q}$-linear implies that the natural map $M^{G} \longrightarrow M / G$, from the invariants to the coinvariants, is an isomorphism, and that the functors $M \mapsto M^{G}$ and $M \mapsto M / G$ preserve weak equivalences, cofibrations and fibrations. (Indeed, if $f: M \longrightarrow M^{\prime}$ is a $G$-equivariant morphism in $\mathfrak{M}$, then $f^{G}: M^{G} \longrightarrow M^{\prime G}$ is a retract of $f$.)

Notation 4.3 - Given a monoidal category with colimits, we denote by $\mathrm{S}^{n}(M)$ the $n$-th symmetric power of an object $M$. Recall that this is given by

$$
\mathrm{S}^{n}(M)=M^{\otimes n} / \Sigma_{n}
$$

The symmetric algebra on $M$ is the $\mathbb{N}$-graded ring $\mathrm{S}(M)=\left\{\mathrm{S}^{n}(M)\right\}_{n \in \mathbb{N}}$.

We fix an object $T \in \mathfrak{M}$. Starting from Defintion 4.10 below, we will assume that $T$ is cofibrant.

Definition $4.4-A$ commutative $T$-spectrum is an $\mathbb{N}$-graded module over the symmetric algebra $\mathrm{S}(T)$.

More explicitly, a commutative T-spectrum $\mathbf{E}$ is a pair $\left(\left\{\mathbf{E}_{n}\right\}_{n \in \mathbb{N}},\left\{\gamma_{n}\right\}_{n \in \mathbb{N}}\right)$ consisting of a collection of objects $\mathbf{E}_{n} \in \mathfrak{M}$ and a collection of maps $\gamma_{n}: T \otimes \mathbf{E}_{n} \longrightarrow \mathbf{E}_{n+1}$, called the assembly maps, satisfying the following condition.

For all $m, n \in \mathbb{N}$, the natural map

$$
\gamma_{m+n-1} \circ \cdots \circ \gamma_{n}: T^{\otimes m} \otimes \mathbf{E}_{n} \longrightarrow \mathbf{E}_{m+n}
$$

is $\Sigma_{m}$-equivariant with respect to the natural action of $\Sigma_{m}$ on $T^{\otimes m}$ and the identity actions on $\mathbf{E}_{n}$ and $\mathbf{E}_{m+n}$. (In particular, this map factors through a $\left.\operatorname{map} \mathrm{S}^{m}(T) \otimes \mathbf{E}_{n} \longrightarrow \mathbf{E}_{m+n}.\right)$

We denote by $\mathbf{S p t}_{T}^{\sharp}(\mathfrak{M})$ the category of commutative T-spectra. This is a symmetric monoidal category with unit.

Remark 4.5 - By construction, $\operatorname{Spt}_{T}^{\sharp}(\mathfrak{M})$ is a full subcategory of $\operatorname{Spt}_{T}(\mathfrak{M})$. Indeed, a graded module over the symmetric algebra on $T$ is naturally a graded left module over the tensor algebra on $T$. Also, $\mathbf{S p t}_{T}^{\sharp}(\mathfrak{M})$ is a full subcategory of $\operatorname{Spt}_{T}^{\Sigma}(\mathfrak{M})$. Indeed, a commutative $T$-spectrum $\mathbf{E}$ becomes a symmetric $T$-spectrum if we endow each $\mathbf{E}_{n}$ with the identity action of $\Sigma_{n}$.

LEMMA 4.6 - The inclusion functor $\operatorname{Spt}_{T}^{\sharp}(\mathfrak{M}) \hookrightarrow \operatorname{Spt}_{T}^{\Sigma}(\mathfrak{M})$ admits a left adjoint

$$
-/ \Sigma: \operatorname{Spt}_{T}^{\Sigma}(\mathfrak{M}) \longrightarrow \operatorname{Spt}_{T}^{\sharp}(\mathfrak{M})
$$

which is monoidal. It sends a symmetric T-spectrum $\mathbf{E}$ to the commutative $T$ spectrum $\mathbf{E} / \Sigma$ given in level $n$ by $(\mathbf{E} / \Sigma)_{n}=\mathbf{E}_{n} / \Sigma_{n}$.

Proof. Given a symmetric $T$-spectrum E, we define a $T$-spectrum $\mathbf{E} / \Sigma$ by setting $(\mathbf{E} / \Sigma)_{n}=\mathbf{E}_{n} / \Sigma_{n}$ and by taking for $\gamma_{n}: T \otimes(\mathbf{E} / \Sigma)_{n} \longrightarrow(\mathbf{E} / \Sigma)_{n+1}$ the composition 
of

$$
T \otimes\left(\mathbf{E}_{n} / \Sigma_{n}\right)=\left(T \otimes \mathbf{E}_{n}\right) /\left(\Sigma_{1} \times \Sigma_{n}\right) \stackrel{\gamma_{n}}{\longrightarrow} \mathbf{E}_{n+1} /\left(\Sigma_{1} \times \Sigma_{n}\right) \rightarrow \mathbf{E}_{n+1} / \Sigma_{n+1} .
$$

Clearly, $\mathbf{E} / \Sigma$ is a commutative $T$-spectrum. Also, given a commutative $T$-spectrum $\mathbf{F}$, every morphism of symmetric $T$-spectra $\mathbf{E} \longrightarrow \mathbf{F}$ factors uniquely through the projection $\mathbf{E} \longrightarrow \mathbf{E} / \Sigma$. This proves that $-/ \Sigma$ is left adjoint to the inclusion functor.

We now check that $-/ \Sigma$ is monoidal. As in [2, Définition 4.3.3], we consider the categories:

$$
\operatorname{Suite}(\mathfrak{M})=\prod_{n \in \mathbb{N}} \mathfrak{M} \quad \text { and } \quad \operatorname{Suite}(\Sigma, \mathfrak{M})=\prod_{n \in \mathbb{N}} \operatorname{Rep}\left(\Sigma_{n}, \mathfrak{M}\right)
$$

Objects in Suite $(\mathfrak{M})$ are simply families $\left(X_{n}\right)_{n \in \mathbb{N}}$ of objects of $\mathfrak{M}$, and objects in Suite $(\Sigma, \mathfrak{M})$ are families $\left(X_{n}\right)_{n \in \mathbb{N}}$ endowed, for each $n \in \mathbb{N}$, with an action of $\Sigma_{n}$ on $X_{n}$. There is an obvious functor

$$
-/ \Sigma: \operatorname{Suite}(\Sigma, \mathfrak{M}) \longrightarrow \operatorname{Suite}(\mathfrak{M})
$$

given by $\left(X_{n}\right)_{n \in \mathbb{N}} \mapsto\left(X_{n} / \Sigma_{n}\right)_{n \in \mathbb{N}}$. Furthermore, the two categories in (16) are symmetric monoidal (see [2, Définition 4.3.63]). In $\operatorname{Suite}(\mathfrak{M})$, the tensor product is given by

$$
\left(X_{n}\right)_{n \in \mathbb{N}} \otimes\left(Y_{n}\right)_{n \in \mathbb{N}}=\left(\coprod_{i+j=n} X_{i} \otimes Y_{j}\right)_{n \in \mathbb{N}} .
$$

In $\operatorname{Suite}(\Sigma, \mathfrak{M})$, the tensor product is given by

$$
\left(X_{n}\right)_{n \in \mathbb{N}} \otimes\left(Y_{n}\right)_{n \in \mathbb{N}}=\left(\coprod_{i+j=n} \operatorname{Ind}_{\Sigma_{i} \times \Sigma_{j}}^{\Sigma_{n}} X_{i} \otimes Y_{j}\right)_{n \in \mathbb{N}} .
$$

From these descriptions, it follows immediately that the functor 17 is symmetric monoidal.

Now, recall that $\operatorname{Spt}_{T}^{\Sigma}(\mathfrak{M})$ can be identified with the category of $\mathrm{S}^{T}$-modules in Suite $(\Sigma, \mathfrak{M})$, where $S^{T}=\left\{T^{\otimes n}\right\}_{n \in \mathbb{N}}$ with $\Sigma_{n}$ is acting on $T^{\otimes n}$ by permutation of factors (see [2, Définition 4.3.68] and [2, Proposition 4.3.70]). Similarly, $\operatorname{Spt}_{T}^{\sharp}(\mathfrak{M})$ can be identified with the category of $\mathrm{S}(T)$-modules in Suite $(\mathfrak{M})$. As $\mathrm{S}(T)=\mathrm{S}^{T} / \Sigma$, there is an induced symmetric monoidal functor

$$
-/ \Sigma: \operatorname{Mod}\left(\mathrm{S}^{T}\right) \longrightarrow \operatorname{Mod}(\mathrm{S}(T)) \text {. }
$$

This finishes the proof of the lemma.

Recall that the $p$-th evaluation functors $\mathbf{E} \mapsto \mathbf{E}_{p}=\operatorname{Ev}_{p}(\mathbf{E})$ admit left adjoints

$$
\operatorname{Sus}_{T}^{p}: \mathfrak{M} \longrightarrow \operatorname{Spt}_{T}(\mathfrak{M}) \quad \text { and } \quad \operatorname{Sus}_{T, \Sigma}^{p}: \mathfrak{M} \longrightarrow \operatorname{Spt}_{T}^{\Sigma}(\mathfrak{M}) .
$$

The same holds for commutative $T$-spectra, and we have the following result.

LEMмA 4.7 - The p-th evaluation functor admits a left adjoint

$$
\operatorname{Sus}_{T, \sharp}^{p}: \mathfrak{M} \longrightarrow \operatorname{Spt}_{T}^{\sharp}(\mathfrak{M}) \text {. }
$$

For $M \in \mathfrak{M}$, we have

$$
\operatorname{Sus}_{T, \sharp}^{p}(M)_{n}=\left\{\begin{array}{cc}
0 & \text { if } n<p, \\
\mathrm{~S}^{n-p}(T) \otimes M & \text { if } n \geqslant p .
\end{array}\right.
$$

We also have the formula

$$
\operatorname{Sus}_{T, \sharp}^{p}(M)=\operatorname{Sus}_{T, \Sigma}^{p}(M) / \Sigma .
$$


Proof. The $p$-th evaluation functor is the composition of

$$
\operatorname{Spt}_{T}^{\sharp}(\mathfrak{M}) \hookrightarrow \operatorname{Spt}_{T}^{\Sigma}(\mathfrak{M}) \stackrel{\mathrm{Ev}_{p}}{\longrightarrow} \mathfrak{M} .
$$

The two functors above have left adjoints given respectively by $-/ \Sigma$ and $\operatorname{Sus}_{T, \Sigma}^{p}$. This gives the existence of $\operatorname{Sus}_{T, \sharp}^{p}$ and the last formula of the statement. The computation of $\operatorname{Sus}_{T, \sharp}^{p}(M)_{n}$ follows then readily from the corresponding formula for $\operatorname{Sus}_{T, \Sigma}^{p}(M)_{n}$ (see [2, Lemme 4.3.9]).

LemMA 4.8 - Let $\mathbf{E}$ be a commutative $T$-spectrum. For $M \in \mathfrak{M}$ and $p \in \mathbb{N}$, we have

$$
\left(\operatorname{Sus}_{T, \sharp}^{p}(M) \otimes \mathbf{E}\right)_{n}=\left\{\begin{array}{cll}
0 & \text { if } & n<p \\
M \otimes \mathbf{E}_{n-p} & \text { if } & n \geqslant p .
\end{array}\right.
$$

Proof. Indeed, as a graded $\mathrm{S}(T)$-module, $\operatorname{Sus}_{T, \sharp}^{p}(M)$ is the free $\mathrm{S}(T)$-module generated by $M$ but whose grading is the $p$-th shift of the natural one. The result follows.

LEMMA 4.9 - For $p, q \in \mathbb{N}$ and $M, N \in \mathfrak{M}$, there is a canonical isomorphism

$$
\operatorname{Sus}_{T, \sharp}^{p}(M) \otimes \operatorname{Sus}_{T, \sharp}^{q}(N) \simeq \operatorname{Sus}_{T, \sharp}^{p+q}(M \otimes N) .
$$

In particular, $\operatorname{Sus}_{T, \sharp}^{0}$ is a monoidal functor.

Proof. This follows from Lemma 4.6 and [2, Corollaire 4.3.72]. Alternatively, one can deduce this from Lemma 4.8.

We now proceed to construct model structures on $\operatorname{Spt}_{T}^{\sharp}(\mathfrak{M})$; for this, we assume that $T$ is cofibrant.

Definition 4.10 - Let $f: \mathbf{E} \longrightarrow \mathbf{F}$ be a morphism of commutative $T$-spectra.

(a) We say that $f$ is a levelwise weak equivalence (resp. cofibration, fibration) if for all $n \in \mathbb{N}$, the map $f_{n}: \mathbf{E}_{n} \longrightarrow \mathbf{F}_{n}$ is a weak equivalence (resp. cofibration, fibration) in $\mathfrak{M}$. We denote by $\mathbf{W}_{\text {level }}$ (resp. $\mathbf{C o f}_{\text {level }}, \mathbf{F i b}_{\text {level }}$ ) the class of those maps.

(b) We say that $f$ is a projective cofibration (resp. injective fibration) if it satisfies the left lifting property with respect to maps in $\mathbf{W}_{\text {level }} \cap \mathbf{F i b}$ level (resp. the right lifting property with respect to maps in $\left.\mathbf{W}_{\text {level }} \cap \mathbf{C o f} \mathbf{f}_{\text {level }}\right)$. We denote by $\mathbf{C o f}_{\text {proj }}$ (resp. $\mathbf{F i b}_{\text {inj }}$ ) the class of those maps.

Proposition 4.11 - The category $\operatorname{Spt}_{T}^{\sharp}(\mathfrak{M})$ admits a projective unstable model structure given by the triple $\left(\mathbf{W}_{\text {level }}, \mathbf{C o f} \mathbf{p r o j}_{\text {provel }}, \mathbf{F i b} \mathbf{b}_{\text {leve }}\right)$ It also admits an injective unstable model structure given by the triple $\left(\mathbf{W}_{\text {level }}, \mathbf{C o f}_{\text {level }}, \mathbf{F i b} \mathbf{b}_{\mathrm{inj}}\right)$. The two model structures are Quillen equivalent and they are both presentable by cofibrations.

Proof. The proof of [2, Proposition 4.3.21] applies mutatis mutandis.

We denote by $\mathbf{H o}_{\text {level }}\left(\mathbf{S p t}_{T}^{\sharp}(\mathfrak{M})\right)$ the homotopy category of the unstable model structures. The projective unstable model structure will be preferred below due to the following result.

Proposition 4.12 - The category $\operatorname{Spt}_{T}^{\sharp}(\mathfrak{M})$ endowed with its projective unstable model structure is a monoidal model category. 
Proof. The proof of [2, Proposition 4.3.75] applies mutatis mutandis.

For later use, we establish the following technical result.

\section{LEMMA 4.13}

(i) Let $Q \in \mathfrak{M}$ be a cofibrant object. If $Q \otimes-$ preserves weak equivalences, then, for every $p \in \mathbb{N}$, $\operatorname{Sus}_{T, \sharp}^{p}(Q) \otimes-$ preserves levelwise weak equivalences.

(ii) If the monoidal model category $\mathfrak{M}$ satisfies the monoid axiom as in [15, Definition 3.3], then so does $\mathbf{S p t}_{T}^{\sharp}(\mathfrak{M})$ endowed with its projective unstable model structure.

Proof. The conclusion of (i) is a direct consequence of Lemma 4.8.

To prove (ii), we need to check that a transfinite compositions of pushouts of maps of the form $c \otimes \mathrm{id}_{\mathbf{E}}: \mathbf{A} \otimes \mathbf{E} \longrightarrow \mathbf{B} \otimes \mathbf{E}$, with $c$ a levelwise trivial projective cofibration, are levelwise weak equivalences. By [15, Lemma 3.5(2)], we may assume that the $c$ 's are obtained form trivial cofibrations of $\mathfrak{M}$ by applying the functors $\operatorname{Sus}_{T, \sharp}^{p}$ 's. As weak equivalences in $\mathfrak{M}$ are assumed to be preserved by filtered colimits, we are left to show that a pushout of

$$
\operatorname{Sus}_{T, \sharp}^{p}(A) \otimes \mathbf{E} \longrightarrow \operatorname{Sus}_{T, \sharp}^{p}(B) \otimes \mathbf{E}
$$

is a levelwise weak equivalence if $A \longrightarrow B$ is a trivial cofibration of $\mathfrak{M}$. This follows from Lemma 4.8 since $\mathfrak{M}$ satisfies the monoid axiom.

Proposition 4.14 - The functor

$$
-/ \Sigma: \operatorname{Spt}_{T}^{\Sigma}(\mathfrak{M}) \longrightarrow \operatorname{Spt}_{T}^{\sharp}(\mathfrak{M})
$$

is a left Quillen functor with respect to the projective (resp. injective) unstable model structures. Moreover, this functor and its right adjoint preserve weak equivalences, levelwise cofibrations and levelwise fibrations.

Proof. By Remark 4.2, the functor $-/ \Sigma$ preserves levelwise weak equivalences, levelwise cofibrations (and levelwise fibrations). This proves that this functor is a left Quillen functor with respect to the injective unstable model structures. On the other hand, the inclusion functor $\operatorname{Spt}_{T}^{\sharp}(\mathfrak{M}) \hookrightarrow \operatorname{Spt}_{T}^{\Sigma}(\mathfrak{M})$ preserves levelwise fibrations. This shows that $-/ \Sigma$ is also left Quillen with respect to the projective unstable model structures. The other statements are obvious.

We now proceed to construct the stable model structures on $\operatorname{Spt}_{T}^{\sharp}(\mathfrak{M})$.

Notation 4.15 — Let $M$ be an object of $\mathfrak{M}$. We have a natural map

$$
\omega_{M}^{p}: \operatorname{Sus}_{T, \sharp}^{p+1}(T \otimes M) \longrightarrow \operatorname{Sus}_{T, \sharp}^{p}(M)
$$

which corresponds by adjunction to the identity map $T \otimes M \simeq \operatorname{Sus}_{T, \sharp}^{p}(M)_{p+1}$.

DEFinition 4.16 - The projective (resp. injective) stable model structure on $\mathbf{S p t}_{T}^{\sharp}(\mathfrak{M})$ is the Bousfield localisation of the projective (resp. injective) unstable model structure with respect to the maps $\omega_{M}^{p}$ for all $p \in \mathbb{N}$ and cofibrant $M \in \mathfrak{M}$. The homotopy category of the stable model structure is denoted by $\mathbf{H o}_{\text {st }}\left(\mathbf{S p t}_{T}^{\sharp}(\mathfrak{M})\right)$.

Remark 4.17 — The existence of the Bousfield localisation in Definition 4.16 can be established by adapting the proof of [2, Lemme 4.3.28]. The two stable model structures on $\operatorname{Spt}_{T}^{\sharp}(\mathfrak{M})$ are presentable by cofibrations. 
Proposition 4.18 - Let $\mathbf{E}$ be a commutative $T$-spectrum. Then $\mathbf{E}$ is projectively stably fibrant (i.e., fibrant with respect to the projective stable model structure) if and only if it satisfies the following two conditions:

(i) $\mathbf{E}$ is levelwise fibrant;

(ii) for every $n \in \mathbb{N}$, the adjoint to the assembly map

$$
\gamma_{n}^{\prime}: \mathbf{E}_{n} \longrightarrow \underline{\operatorname{Hom}}\left(T, \mathbf{E}_{n+1}\right)
$$

is a weak equivalence.

Proof. The proof of [2, Proposition 4.3.30] applies mutatis mutandis.

Remark 4.19 - Recall that a $T$-spectrum $\mathbf{E}$ is called an $\Omega$-spectrum if, for all $n \in \mathbb{N}$, the map $\gamma_{n}^{\prime}: \mathbf{E}_{n} \longrightarrow \operatorname{R} \underline{\operatorname{Hom}}\left(T, \mathbf{E}_{n+1}\right)$ is an isomorphism in $\mathbf{H o}(\mathfrak{M})$. Thus, a commutative $T$-spectrum is projectively stably fibrant if and only if it is a levelwise fibrant $\Omega$-spectrum.

Proposition 4.20 - Assume that the category $\mathbf{S p t}_{T}^{\sharp}(\mathfrak{M})$ endowed with its stable model structure is stable in the sense of [9, Definition 7.1.1]. Then, the category $\mathbf{S p t}_{T}^{\sharp}(\mathfrak{M})$ endowed with its projective stable model structure is a monoidal model category.

Proof. The claim follows from Proposition 4.12 in the same way that [2, Théorème 4.3.76] follows from [2, Proposition 4.3.75].

If the model category $\mathfrak{M}$ is stable, then $\operatorname{Spt}_{T}^{\sharp}(\mathfrak{M})$ is also stable. In fact, much less is needed as the following result shows.

LEMMA 4.21 - The projective stable model structure on $\mathbf{S p t}_{T}^{\sharp}(\mathfrak{M})$ is stable in the sense of [9, Definition 7.1.1] if the object $T \in \mathbf{H o}(\mathfrak{M})$ is isomorphic to a suspension.

Proof. The proof of [2, Proposition 4.3.77] applies mutatis mutandis.

For later use, we establish the following technical result.

LEMMA 4.22 - We assume the following conditions.

- The category $\operatorname{Spt}_{T}^{\sharp}(\mathfrak{M})$ endowed with its stable model structure is stable in the sense of [9, Definition 7.1.1].

- The endofunctor $Q \otimes$ - preserves weak equivalences for every cofibrant object $Q \in \mathfrak{M}$.

- For every diagram in $\mathfrak{M}$ of the form

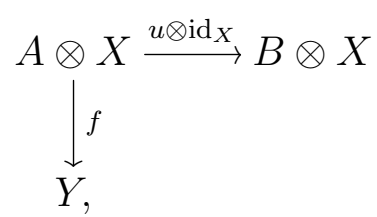

with $u: A \longrightarrow B$ a cofibration, the colimit is naturally isomorphic to the homotopy colimit in $\mathbf{H o}(\mathfrak{M})$.

Then, the following properties hold.

(i) For every projectively cofibrant commutative T-spectrum $\mathbf{E}$, the endofunctor $\mathbf{E} \otimes-$ preserves stable weak equivalences. 
(ii) If the monoidal model category $\mathfrak{M}$ satisfies the monoid axiom as in [15, Definition 3.3], then so does $\mathbf{S p t}_{T}^{\sharp}(\mathfrak{M})$ endowed with its projective stable model structure.

Proof. We split the proof in two parts.

Part 1: Here we prove (i). By Lemma 4.13(i), $\operatorname{Sus}_{T, \sharp}^{p}(Q) \otimes-$ preserves levelwise weak equivalences for every cofibrant object $Q \in \mathfrak{M}$. Since, $\operatorname{Sus}_{T, \sharp}^{p}(Q) \otimes-$ is also a left Quillen functor with respect to the projective stable model structure, we deduce that it preserves stable weak equivalences.

Now, recall that a projectively cofibrant commutative $T$-spectrum $\mathbf{E}$ is a retract of the target of a map from 0 which is a transfinite composition of pushouts of maps of the form $\operatorname{Sus}_{T, \sharp}^{p}(A) \longrightarrow \operatorname{Sus}_{T, \sharp}^{p}(B)$, with $p \in \mathbb{N}$ and $A \longrightarrow B$ a cofibration of $\mathfrak{M}$. As filtered colimits preserve stable equivalences, it is enough to show the following property: if $\mathbf{E}$ is a projectively cofibrant commutative $T$-spectrum satisfying the conclusion of (i), then so does the colimit of

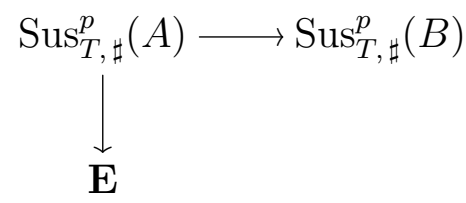

for every cofibration $A \longrightarrow B$. Let $\mathbf{F}$ be this colimit and set $C=B / A$. We thus have a distinguished triangle in the triangulated category $\mathbf{H o}_{\mathrm{st}}\left(\mathbf{S p t}_{T}^{\sharp}(\mathfrak{M})\right)$ :

$$
\mathbf{E} \longrightarrow \mathbf{F} \longrightarrow \operatorname{Sus}_{T, \sharp}^{p}(C) \longrightarrow \text {. }
$$

We need to show that the endofunctor $\mathbf{F} \otimes-$ preserves stable equivalences. It is enough to show that

$$
\mathbf{F} \stackrel{\mathrm{L}}{\otimes} \mathbf{G} \longrightarrow \mathbf{F} \otimes \mathbf{G}
$$

is an isomorphism in $\mathbf{H o}_{\text {st }}\left(\mathbf{S p t}_{T}^{\sharp}(\mathfrak{M})\right)$ for every commutative $T$-spectrum G. Clearly, $\mathbf{F} \stackrel{\mathrm{L}}{\otimes} \mathbf{G}$ is the homotopy colimit of

$$
\begin{aligned}
& \operatorname{Sus}_{T, \sharp}^{p}(A) \stackrel{\mathrm{L}}{\otimes} \mathbf{G} \longrightarrow \operatorname{Sus}_{T, \sharp}^{p}(B) \stackrel{\mathrm{L}}{\otimes} \mathbf{G} \\
& \mathbf{E} \stackrel{\mathrm{L}}{\otimes} \mathbf{G} \text {. }
\end{aligned}
$$

By our assumption about the endofunctor $\mathbf{E} \otimes-$, the natural morphism

$$
\mathbf{E} \otimes \mathbf{L} \mathbf{G} \longrightarrow \mathbf{E} \otimes \mathbf{G}
$$

is an isomorphism in $\mathbf{H o}_{\mathrm{st}}\left(\mathbf{S p t}_{T}^{\sharp}(\mathfrak{M})\right)$. On the other hand, the square

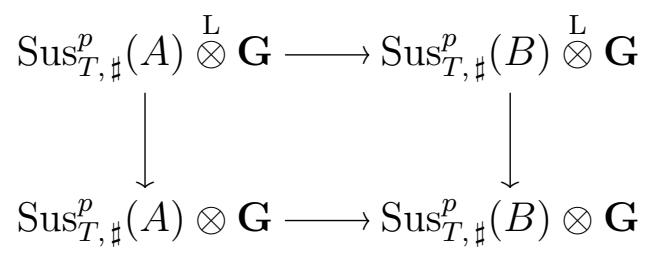

is homotopy cocartesian in the triangulated category $\mathbf{H o}_{\text {st }}\left(\mathbf{S p t}_{T}^{\sharp}(\mathfrak{M})\right)$. Indeed, taking the homotopy cofiber of the upper horizontal map gives $\operatorname{Sus}_{T, \sharp}^{p}(C) \stackrel{\mathrm{L}}{\otimes} \mathbf{G}$. On the 
other hand, using Lemma 4.8 and the third condition of the statement, the homotopy cofiber of the lower horizontal map is isomorphic to $\operatorname{Sus}_{T, \sharp}^{p}(C) \otimes \mathbf{G}$. (Indeed, homotopy cofiber can be computed using the injective unstable model structure.) Since $C$ is cofibrant, we know from the beginning of the proof, that

$$
\operatorname{Sus}_{T, \sharp}^{p}(C) \stackrel{\mathrm{L}}{\otimes} \mathbf{G} \longrightarrow \operatorname{Sus}_{T, \sharp}^{p}(C) \otimes \mathbf{G}
$$

is an isomorphism in $\mathbf{H o}_{\text {st }}\left(\mathbf{S p t}_{T}^{\sharp}(\mathfrak{M})\right)$.

Putting everything together, we see that the homotopy pushout of $(19)$ is naturally equivalent to the homotopy colimit of

$$
\begin{aligned}
& \operatorname{Sus}_{T, \sharp}^{p}(A) \otimes \mathbf{G} \longrightarrow \operatorname{Sus}_{T, \sharp}^{p}(B) \otimes \mathbf{G} \\
& \mathbf{E} \otimes \mathbf{G}
\end{aligned}
$$

which, by Lemma 4.8 and the third condition of the statement, is isomorphic to $\mathbf{F} \otimes \mathbf{G}$ in $\mathbf{H o}_{\text {st }}\left(\mathbf{S p t}_{T}^{\sharp}(\mathfrak{M})\right)$. This finishes the proof of (i).

Part 2: Here we prove (ii). Arguing as in the proof of Lemma 4.13(ii), we are left to show the following property: given a commutative $T$-spectrum $\mathbf{E}$, a cofibrant object $M \in \mathfrak{M}$ and a factorisation

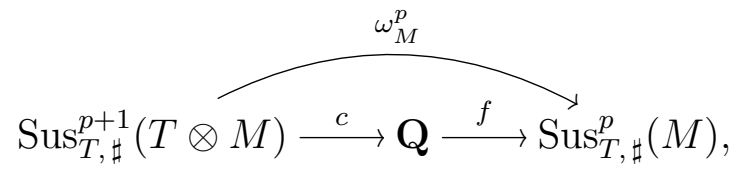

with $c$ is a projective cofibration and $f$ is a levelwise weak equivalence, every pushout of

$$
\operatorname{Sus}_{T, \sharp}^{p+1}(T \otimes M) \otimes \mathbf{E} \longrightarrow \mathbf{Q} \otimes \mathbf{E}
$$

is a stable weak equivalence.

The third condition of the statement implies an analogous property on commutative $T$-spectra. More precisely, given a projective cofibration $\mathbf{A} \longrightarrow \mathbf{B}$ and a commutative $T$-spectrum $\mathbf{X}$, every pushout of

$$
\mathbf{A} \otimes \mathbf{X} \longrightarrow \mathbf{B} \otimes \mathbf{X}
$$

is isomorphic to the corresponding homotopy pushout in $\mathbf{H o}_{\text {level }}\left(\mathbf{S p t}_{T}^{\sharp}(\mathfrak{M})\right)$. Indeed, using that a projective cofibration is a retract of a transfinite composition of pushouts of maps of the form $\operatorname{Sus}_{T, \sharp}^{p}(A) \longrightarrow \operatorname{Sus}_{T, \sharp}^{p}(B)$, with $p \in \mathbb{N}$ and $A \longrightarrow B$ a cofibration of $\mathfrak{M}$, and that filtered colimits preserve levelwise weak equivalences, we are left to treat the case of $\operatorname{Sus}_{T, \sharp}^{p}(A) \longrightarrow \operatorname{Sus}_{T, \sharp}^{p}(B)$. By Lemma 4.8, this follows from the third condition of the statement.

We now get back to the proof of (ii). By the previous discussion, every pushout of (20) is actually a homotopy pushout with respect to the unstable model structure. Therefore, it is enough to show that 20 is a stable equivalence. Since $\operatorname{Sus}_{T, \sharp}^{p+1}(T \otimes M)$ and $\mathbf{Q}$ are both projectively cofibrant, part (i) of the lemma (which we proved above) shows that 20 can be identified with

$$
\operatorname{Sus}_{T, \sharp}^{p+1}(T \otimes M) \stackrel{\mathrm{L}}{\otimes} \mathbf{E} \longrightarrow \mathbf{Q} \stackrel{\mathrm{L}}{\otimes} \mathbf{E}
$$

in $\mathbf{H o}_{\text {st }}\left(\mathbf{S p t}_{T}^{\sharp}(\mathfrak{M})\right)$. This finishes the proof since the endofunctor $-\stackrel{\mathrm{L}}{\otimes} \mathbf{E}$ preserves stable equivalences. 
Proposition 4.23 - The adjunction

$$
(-/ \Sigma, \iota): \operatorname{Spt}_{T}^{\Sigma}(\mathfrak{M}) \longrightarrow \operatorname{Spt}_{T}^{\sharp}(\mathfrak{M})
$$

is a Quillen adjunction with respect to the projective (resp. injective) stable model structures. Moreover, the functor $-/ \Sigma$ preserves stable weak equivalences, so that it derives trivially. Also, the right derived functor

$$
\mathrm{R} \iota: \mathbf{H o}_{\mathrm{st}}\left(\mathbf{S p t}_{T}^{\sharp}(\mathfrak{M})\right) \longrightarrow \mathbf{H o}_{\mathrm{st}}\left(\mathbf{S p t}_{T}^{\Sigma}(\mathfrak{M})\right)
$$

is fully faithful.

Proof. Except the fully faithfulness of R $\iota$, this follows from Proposition 4.14, and the fact that

identifies with

$$
\omega_{M}^{p} / \Sigma: \operatorname{Sus}_{T, \Sigma}^{p+1}(T \otimes M) / \Sigma \longrightarrow \operatorname{Sus}_{T, \Sigma}^{p}(M) / \Sigma
$$

$$
\omega_{M}^{p}: \operatorname{Sus}_{T, \sharp}^{p+1}(T \otimes M) \longrightarrow \operatorname{Sus}_{T, \sharp}^{p}(M)
$$

by Lemma 4.7. To prove the last claim, we need to check that the counit of the adjunction $(-/ \Sigma, \mathrm{R} \iota)$ is an isomorphism. Let $\mathbf{E}$ be a projectively stably fibrant commutative $T$-spectrum so that $\operatorname{R} \iota(\mathbf{E})$ is levelwise weakly equivalent to $\mathbf{E}$. In this case, the counit map can be identified with the identity map id $: \mathbf{E} / \Sigma \longrightarrow \mathbf{E}$. This proves the claim.

We now arrive at the main result of this section.

THEOREM 4.24 - We assume that the following conditions are satisfied.

(i) The transposition $\tau \in \Sigma_{2}$ acts by identity on $T^{\otimes 2}$ in $\mathbf{H o}(\mathfrak{M})$ (i.e., that $T \in$ $\operatorname{Ho}(\mathfrak{M})$ is even of dimension 1 in the sense of Kimura).

(ii) The functor $\mathrm{RHom}(T,-)$ commutes with filtered colimits in $\mathfrak{M}$.

(iii) The object $T$ is isomorphic to a suspension in $\mathbf{H o}(\mathfrak{M})$.

Then, the functor

$$
-/ \Sigma: \operatorname{Spt}_{T}^{\Sigma}(\mathfrak{M}) \longrightarrow \operatorname{Spt}_{T}^{\sharp}(\mathfrak{M})
$$

is a left Quillen equivalence with respect to the projective stable model structures.

Proof. By Proposition 4.23, we already know that $-/ \Sigma$ is a left Quillen functor and that $\mathrm{R} \iota$ is fully faithful. Thus, it remains to show that $\mathrm{R} \iota$ is essentially surjective. Condition (i) implies that the cyclic permutation (123) $\in \Sigma_{3}$ acts also by identity on $T^{\otimes 3}$ in $\mathbf{H o}(\mathfrak{M})$. Thus, by [2, Théorème 4.3.79], the forgetful functor

$$
\mathrm{Oub}^{\Sigma}: \mathbf{S p t}_{T}^{\Sigma}(\mathfrak{M}) \longrightarrow \mathbf{S p t}_{T}(\mathfrak{M})
$$

is a right Quillen equivalence. Therefore, it is enough to show that the composition of

$$
\mathbf{H o}_{\text {st }}\left(\mathbf{S p t}_{T}^{\sharp}(\mathfrak{M})\right) \stackrel{\mathrm{R} \iota}{\longrightarrow} \mathbf{H o}_{\text {st }}\left(\mathbf{S p t}_{T}^{\Sigma}(\mathfrak{M})\right) \stackrel{\mathrm{ROub}^{\Sigma}}{\longrightarrow} \mathbf{H o}_{\text {st }}\left(\mathbf{S p t}_{T}(\mathfrak{M})\right)
$$

is essentially surjective.

Now, by condition (i), Lemma 4.21, [2, Proposition 4.3.77] and [2, Théorème 4.3.79], the functors $\mathrm{R} \iota$ and $\mathrm{ROub}^{\Sigma}$ are triangulated functors. Also, they commute with infinite sums. (Indeed, by condition (ii), infinite direct sums preserve $\Omega$-spectra and $\mathrm{R} \iota$ and $\mathrm{ROub}^{\Sigma}$ can be computed on $\Omega$-spectra without a further fibrant replacement.) As $\mathbf{H o}_{\text {st }}\left(\mathbf{S p t}_{T}(\mathfrak{M})\right)$ coincides with its smallest triangulated subcategory closed under direct sums and containing the objects $\operatorname{Sus}_{T}^{p}(M)$ for $p \in \mathbb{N}$ and $M \in \mathfrak{M}$ cofibrant, it is enough to show that the $\operatorname{Sus}_{T}^{p}(M)$ 's belong to the image 
of the composition of (21). More precisely, we will show that the natural morphism of $T$-spectra

$$
\operatorname{Sus}_{T}^{p}(M) \longrightarrow \operatorname{Sus}_{T, \sharp}^{p}(M)
$$

is a stable weak equivalence in $\operatorname{Spt}_{T}(\mathfrak{M})$. In fact, this is a levelwise weak equivalence since, in degree $n \geqslant p,(22)$ is given by

$$
T^{\otimes n-p} \otimes M \longrightarrow \mathrm{S}^{n-p}(T) \otimes M,
$$

and condition (i) of the statement insures that $\Sigma_{n-p}$ is acting by identity on $T^{\otimes n-p}$ in $\mathbf{H o}(\mathfrak{M})$ so that $T^{\otimes n-p} / \Sigma_{n-p}$ is weakly equivalent to $T^{\otimes n-p}$.

In this paper, we will use the following model for $\mathbf{D} \mathbf{A}^{\text {ét }}(S ; \Lambda)$, the triangulated category of motives.

Definition 4.25 - Assume that $\Lambda$ is a $\mathbb{Q}$-algebra. Let $S$ be a base scheme of characteristic zero (i.e., $S$ is a $\mathbb{Q}$-scheme). Using the notation as in [2, §4.4.1], we set

$$
T_{S}=\left(\mathbb{P}_{S}^{1}, \infty_{S}\right) \otimes \Lambda=\left(\mathbb{P}_{S}^{1} \otimes \Lambda\right) /\left(\infty_{S} \otimes \Lambda\right)
$$

this is a presheaf of $\Lambda$-modules on $\mathrm{Sm} / S$. The model category

$$
\operatorname{Spt}_{T_{S}}^{\sharp}(\mathbf{C p l}(\mathbf{P S h}(\operatorname{Sm} / S ; \Lambda)))
$$

will be endowed with its stable ( $\mathbb{A}^{1}$, ét)-local model structure. More precisely, this is the projective stable model category on commutative $T_{S^{-}}$spectra as in Definition 4.16 deduced from the projective ( $\mathbb{A}^{1}$, ét)-local model structure on $\mathbf{C p l}(\mathbf{P S h}(\operatorname{Sm} / S ; \Lambda))$ as in [2, Définition 4.5.12] (see also the beginning of [4, §3] for a more concise discussion). We set

$$
\mathbf{D A}^{\text {ét }}(S ; \Lambda)=\mathbf{H o}_{\text {st }}\left(\mathbf{S p t}_{T_{S}}^{\sharp}(\mathbf{C p l}(\mathbf{P S h}(\operatorname{Sm} / S ; \Lambda)))\right) .
$$

Remark 4.26 - Up to a (monoidal triangulated) equivalence, the category $\mathbf{D A}^{\text {ét }}(S ; \Lambda)$ as defined above coincides with the categories described in [2, Définition 4.5.21] (for $\mathfrak{M}=\operatorname{Cpl}(\Lambda)$ and $\tau=$ ét) and at the beginning of [4, §3]. This follows from Theorem 4.24. Indeed, $T_{S} \in \mathbf{D A}^{\text {eff, ét }}(S ; \Lambda)$ is even of dimension 1 as it follows from the equivalence of categories $\mathbf{D} \mathbf{A}^{\text {eff, ét }}(S ; \Lambda) \simeq \mathbf{D M}^{\text {eff, ét }}(S ; \Lambda)$ of $[5$, Théorème B.1], available for $S$ of characteristic zero, and the well known fact that $\mathbb{Z}(1) \in$ $\mathbf{D M}^{\text {eff }}(k, \mathbb{Z})$ is even of dimension 1 for any field $k$. (The last property follows from [16. Lemma 4.8]; see also [17, Chapter 5, Corollary 2.1.5] and [11, Proposition 15.7].7]

LEMMA 4.27 - The category $\operatorname{Spt}_{T_{S}}^{\sharp}(\mathbf{C p l}(\mathbf{P S h}(\operatorname{Sm} / S ; \Lambda)))$, endowed with its stable $\left(\mathbb{A}^{1}\right.$, ét)-local model structure, is a monoidal model category satisfying the monoid axiom as in [15, Definition 3.3]. Moreover, for every projectively cofibrant commutative $T_{k}$-spectrum $\mathbf{E}$, the endofunctor $\mathbf{E} \otimes-$ preserves stable ( $\mathbb{A}^{1}$, ét)-local equivalences.

Proof. This follows immediately from Lemma 4.22 .

\footnotetext{
${ }^{7}$ Unfortunately, the proof in [1] is incomplete: it assumes wrongly that permutation matrices belong to $S L_{n}(k)$.
} 


\section{Stabilis ATiOn OF COMmUtative SPECTRA}

The goal of this section is to describe an explicit way to turn a commutative $T$-spectrum into an $\Omega$-spectrum. The story will be parallel to that for ordinary $T$-spectra which was detailed in [2, §4.3.4]. As in Section $4 .(\mathfrak{M}, \otimes, \mathbf{1})$ is a monoidal model category with cofibrant unit and $T \in \mathfrak{M}$ is a cofibrant object. We will work under the following assumptions.

\section{Hypothesis 5.1}

- The model category $\mathfrak{M}$ is presentable by cofibrations as in [2, Définition 4.2.39]. (Another possibility is to assume that $\mathfrak{M}$ is cellular as in [8, Definition 12.1.1].)

- Coproducts and filtered colimits in $\mathfrak{M}$ preserve weak equivalences.

- The category $\mathfrak{M}$ is additive and $\mathbb{Q}$-linear.

- The functor $\mathrm{R} \underline{\mathrm{Hom}}(T,-)$ commutes with filtered colimits in $\mathfrak{M}$.

- The transposition $\tau \in \Sigma_{2}$ acts by identity on $T^{\otimes 2}$ in $\mathbf{H o}(\mathfrak{M})$.

We start with the following lemma (which is the analogue of [2, Lemme 4.3.59]).

LEMMA $5.2-$ Let $f: \mathbf{E} \longrightarrow \mathbf{F}$ be a morphism of commutative T-spectra. Assume that $f_{n}: \mathbf{E}_{n} \longrightarrow \mathbf{F}_{n}$ is a weak equivalence for $n \geqslant N$. Then $f$ is a stable equivalence.

Proof. We argue by induction on $N$. When $N=0$, there is nothing to prove. Thus, we assume that $N \geqslant 1$.

We may assume that $f$ is a levelwise cofibration and that $\mathbf{E}$ and $\mathbf{F}$ are levelwise cofibrant. We may also assume that $\mathbf{E}_{n}=0$ for $n<N$. Then, by replacing $\mathbf{E}$ by $\mathbf{E} \amalg_{\operatorname{Sus}_{T, \sharp}^{N}\left(\mathbf{E}_{N}\right)} \operatorname{Sus}_{T, \sharp}^{N}\left(\mathbf{F}_{N}\right)$, we may assume that $\mathbf{E}_{N}=\mathbf{F}_{N}$. (At this point, we loose the property that $f$ is levelwise cofibrant.)

By adjunction, the map $\gamma_{N-1}: T \otimes \mathbf{F}_{N-1} \longrightarrow \mathbf{F}_{N}=\mathbf{E}_{N}$ gives rise to a morphism of commutative $T$-spectra

$$
a: \operatorname{Sus}_{T, \sharp}^{N}\left(T \otimes \mathbf{F}_{N-1}\right) \longrightarrow \mathbf{E} .
$$

Moreover, the square

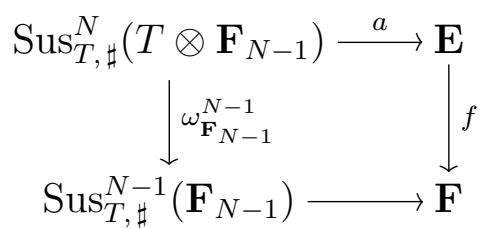

is commutative. Let $\mathbf{G}$ be the homotopy pushout of the diagram

$$
\begin{gathered}
\mathbf{A}=\operatorname{Sus}_{T, \sharp}^{N}\left(T \otimes \mathbf{F}_{N-1}\right) \stackrel{a}{\longrightarrow} \mathbf{E} \\
\underset{\mid}{\omega_{\mathbf{F}_{N-1}^{N-1}}^{N}} \\
\mathbf{B}=\operatorname{Sus}_{T, \sharp}^{N-1}\left(\mathbf{F}_{N-1}\right) .
\end{gathered}
$$

Then clearly, the map $\mathbf{E} \longrightarrow \mathbf{G}$ is a stable equivalence. Thus, it is enough to show that $\mathbf{G} \longrightarrow \mathbf{F}$ is a stable equivalence. Now, the assumption that $\tau \in \Sigma_{2}$ acts by identity on $T^{\otimes 2}$ in $\mathbf{H o}(\mathfrak{M})$ implies that $\mathbf{A}_{n} \longrightarrow \mathbf{B}_{n}$ is a weak equivalence for $n \geqslant N$. This, in turn, implies that $\mathbf{G}_{n} \longrightarrow \mathbf{F}_{n}$ is a weak equivalence for $n \geqslant N$. On the other hand, by construction, $\mathbf{G}_{N-1} \simeq \mathbf{F}_{N-1}$. Thus, in fact, $\mathbf{G}_{n} \longrightarrow \mathbf{F}_{n}$ is 
a weak equivalence for $n \geqslant N-1$. We may now use the induction hypothesis to conclude.

Notation 5.3 - Let $\mathbf{E}$ be a $T$-spectrum and let $M$ be an object of $\mathfrak{M}$. We denote by $M \otimes \mathbf{E}$ the $T$-spectrum given by $(M \otimes \mathbf{E})_{n}=M \otimes \mathbf{E}_{n}$, for $n \in \mathbb{N}$, and whose assembly map at level $n$ is given by the composition of

$$
T \otimes M \otimes \mathbf{E}_{n} \stackrel{\tau}{\sim} M \otimes T \otimes \mathbf{E}_{n} \stackrel{\operatorname{id} \otimes \gamma_{n}}{\longrightarrow} M \otimes \mathbf{E}_{n+1} .
$$

This defines an endofunctor

$$
M \otimes-: \mathbf{S p t}_{T}(\mathfrak{M}) \longrightarrow \mathbf{S p t}_{T}(\mathfrak{M}) .
$$

It admits a right adjoint which we denote by $\underline{\operatorname{Hom}}(M,-)$. The $T$-spectrum $\underline{\operatorname{Hom}}(M, \mathbf{E})$ can be described as follows. For $n \in \mathbb{N}$, we have $\underline{\operatorname{Hom}}(M, \mathbf{E})_{n}=\underline{\operatorname{Hom}}\left(M, \mathbf{E}_{n}\right)$ and the adjoint of the assembly map at level $n$ is the composition of

$$
\underline{\operatorname{Hom}}\left(M, \mathbf{E}_{n}\right) \stackrel{\gamma_{n}^{\prime}}{\longrightarrow} \underline{\operatorname{Hom}}\left(M, \underline{\operatorname{Hom}}\left(T, \mathbf{E}_{n+1}\right)\right) \stackrel{\tau}{\sim} \underline{\operatorname{Hom}}\left(T, \underline{\operatorname{Hom}}\left(M, \mathbf{E}_{n+1}\right)\right) .
$$

Clearly, if $\mathbf{E}$ is a commutative $T$-spectrum, then $M \otimes \mathbf{E}$ and $\underline{\operatorname{Hom}}(M, \mathbf{E})$ are also commutative. Thus, we have a pair of adjoint endofunctors

$$
(M \otimes-, \underline{\operatorname{Hom}}(M,-)): \mathbf{S p t}_{T}^{\sharp}(\mathfrak{M}) \longrightarrow \mathbf{S p t}_{T}^{\sharp}(\mathfrak{M})
$$

commuting with the inclusion $\operatorname{Spt}_{T}^{\sharp}(\mathfrak{M}) \hookrightarrow \operatorname{Spt}_{T}(\mathfrak{M})$.

Proposition 5.4 - Let $M \in \mathfrak{M}$ be a cofibrant object. Assume that the endofunctor $\mathrm{RHom}(M,-)$ of $\mathbf{H o}(\mathfrak{M})$ commutes with filtered colimits. Then, the endofunctor $\operatorname{RHom}(M,-)$ of $\mathbf{H o}_{\text {level }}\left(\mathbf{S p t}^{\sharp}(\mathfrak{M})\right)$ preserves stable equivalences.

Proof. The proof is similar to that of [2, Proposition 4.3.57]. As in loc. cit., we reduce to showing that $\operatorname{RHom}(M, f)$ is a stable equivalence when $f$ is a homotopy pushout of $\omega_{N}^{p}: \operatorname{Sus}_{T, \sharp}^{p+1}(T \otimes N) \longrightarrow \operatorname{Sus}_{T, \sharp}^{p}(N)$, with $N \in \mathfrak{M}$ a cofibrant object. As $\Sigma_{n}$ is acting by identity on $T^{\otimes n}$ in $\mathbf{H o}(\mathfrak{M})$, the map $f_{n}$ is a weak equivalence for all $n \geqslant p+1$. This implies that $\underline{\operatorname{RHom}}(M, f)_{n}$ is also a weak equivalence for $n \geqslant p+1$. By Lemma 5.2 , it follows that $\underline{\operatorname{Rom}}(M, f)$ is a stable equivalence.

Notation 5.5 - Recall that we have an adjunction $\left(\mathrm{s}_{+}, \mathrm{s}_{-}\right)$where $\mathrm{s}_{+}$and $\mathrm{s}_{-}$are endofunctors of $\mathbf{S p t}_{T}(\mathfrak{M})$ given as follows. If $\mathbf{E}$ is a $T$-spectrum, $\left(\mathrm{s}_{-}(\mathbf{E})\right)_{n}=\mathbf{E}_{n+1}$ for $n \geqslant 0,\left(\mathrm{~s}_{+}(\mathbf{E})\right)_{n}=\mathbf{E}_{n-1}$ for $n \geqslant 1$ and $\left(\mathrm{s}_{+}(\mathbf{E})\right)_{0}=0$. Clearly, these functors preserve commutative $T$-spectra. Thus, we get also an adjunction

$$
\left(\mathrm{s}_{+}, \mathrm{s}_{-}\right): \operatorname{Spt}_{T}^{\sharp}(\mathfrak{M}) \longrightarrow \operatorname{Spt}_{T}^{\sharp}(\mathfrak{M})
$$

and both functors commute with the inclusion $\operatorname{Spt}_{T}^{\sharp}(\mathfrak{M}) \hookrightarrow \operatorname{Spt}_{T}(\mathfrak{M})$.

The next lemma fails for $T$-spectra which are not commutative.

LEMMA 5.6 - Let $\mathbf{E}$ be a commutative T-spectrum. There is a natural morphism of commutative T-spectra

$$
T \otimes \mathbf{E} \longrightarrow \mathrm{s}_{-}(\mathbf{E})
$$

which is given in level $n$ by the assembly map $\gamma_{n}: T \otimes \mathbf{E}_{n} \longrightarrow \mathbf{E}_{n+1}$. 
Proof. We need to show that the following diagram is commutative

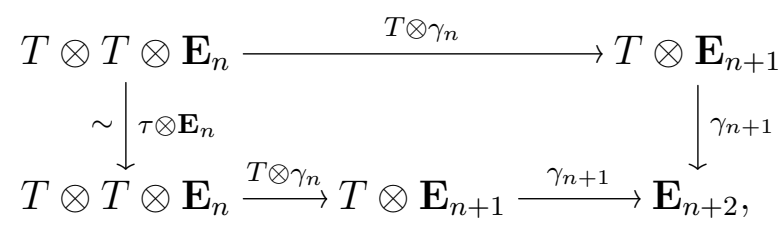

which is clearly true for a commutative $T$-spectrum.

Construction 5.7 - Let $\mathbf{E}$ be a commutative $T$-spectrum. We set

$$
\boldsymbol{\Lambda}(\mathbf{E})=\mathrm{s}_{-} \underline{\operatorname{Hom}}(T, \mathbf{E})=\underline{\operatorname{Hom}}\left(T, \mathrm{~s}_{-} \mathbf{E}\right) .
$$

This defines an endofunctor

$$
\Lambda: \operatorname{Spt}_{T}^{\sharp}(\mathfrak{M}) \longrightarrow \operatorname{Spt}_{T}^{\sharp}(\mathfrak{M}) .
$$

Moreover, by adjunction, 23 induces a natural transformation

$$
\lambda: \text { id } \longrightarrow \Lambda \text {. }
$$

For a commutative $T$-spectrum $\mathbf{E}, \lambda_{\mathbf{E}}: \mathbf{E} \longrightarrow \boldsymbol{\Lambda}(\mathbf{E})$ is given, in level $n$, by the adjoint to the assembly map $\gamma_{n}^{\prime}: \mathbf{E}_{n} \longrightarrow \underline{\operatorname{Hom}}\left(T, \mathbf{E}_{n+1}\right)$.

THEOREM 5.8 - Let $\mathbf{E}$ be a commutative T-spectrum which we assume to be levelwise fibrant. The morphism $\lambda_{\mathbf{E}}: \mathbf{E} \longrightarrow \boldsymbol{\Lambda}(\mathbf{E})$ is a stable equivalence.

Proof. By Proposition 5.4 the endofunctor $\operatorname{RHom}(T,-)$ of $\mathbf{H o}_{\text {level }}\left(\mathbf{S p t}_{T}^{\sharp}(\mathfrak{M})\right)$ preserves stable weak equivalences. The same is true for $\mathrm{Rs}_{-}$. (The proof of this uses the same method as the proof of Proposition 5.4, we leave the details to the reader.) Thus, $\mathrm{R} \Lambda$ preserves also stable equivalences. Therefore, to prove the theorem, we may assume that $\mathbf{E}$ is projectively stably fibrant. In this case $\lambda_{\mathbf{E}}$ is a levelwise weak equivalence by Proposition 4.18.

Remark 5.9 - The next proposition is analogous to [5, Lemme 2.40]. We warn the reader that the two statements are formally incompatible! In fact, the statement of [5. Lemme 2.40] is wrong: the definition of the isomorphism

$$
\tau_{(n)}: \boldsymbol{\Lambda}^{n+1}(\mathbf{E}) \stackrel{\sim}{\longrightarrow} \Lambda^{n+1}(\mathbf{E})
$$

needs to be changed so that the triangle commutes. More precisely, in loc. cit., the action of the permutation $\tau_{(n)}$ on the functor

$$
\mathrm{S}_{-}^{\mathrm{on+1}} \underline{\operatorname{hom}}\left(\left(\mathbb{P}^{1, \text { an }}, \infty\right)^{\wedge n+1},-\right)
$$

should be defined as the composition of the action of $\tau_{(n)}$ on $\mathrm{s}_{-}^{\circ n+1}$ with the inverse of the natural transformation $\underline{\operatorname{hom}}\left(\tau_{(n)},-\right)$, where $\tau_{(n)}$ is acting by permuting the factors of $\left(\mathbb{P}^{1, \text { an }}, \infty\right)^{\wedge n+1}$. Fortunately, this has no consequence on the proof of $[5$, Théorème 2.37] which only uses the existence of a commutative triangle as in the statement of [5. Lemme 2.40].

Proposition 5.10 - For a commutative T-spectrum E, the triangle

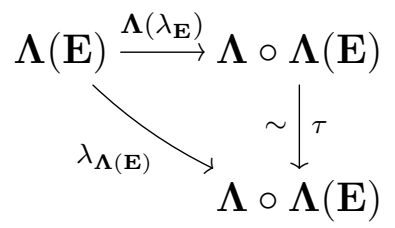


commutes.

Proof. In level $n \in \mathbb{N}$, this triangle looks like

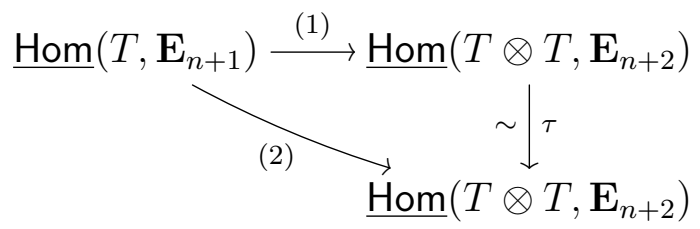

where (1) and (2) are described as follows. By construction, the map (1) is the composition of

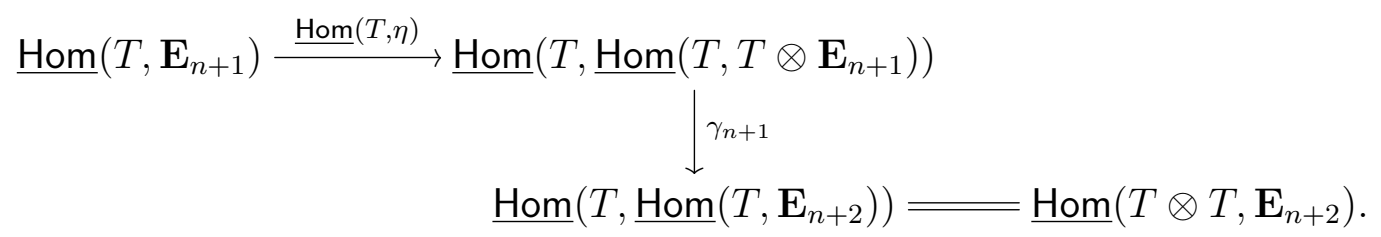

Similarly, by construction, the map (2) is the composition of

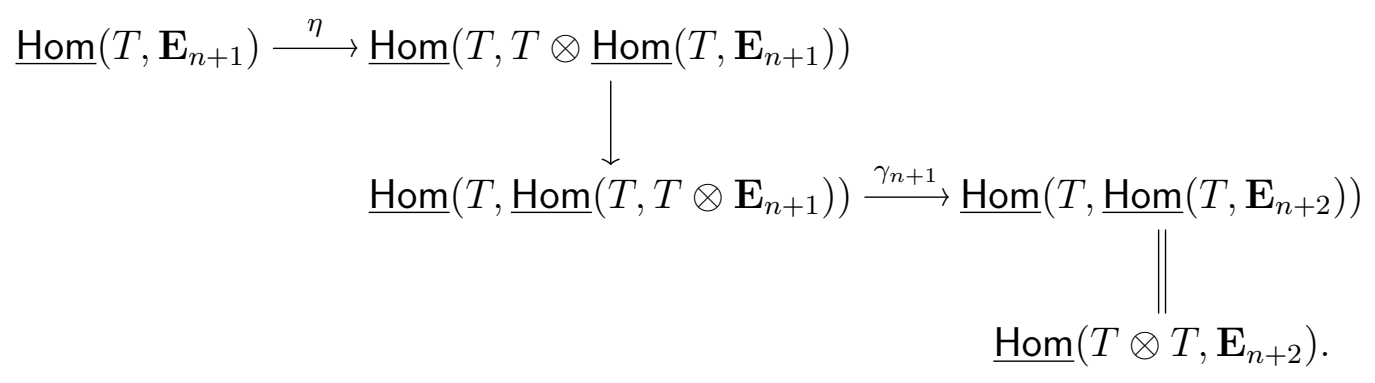

Thus, it is enough to show that the diagram

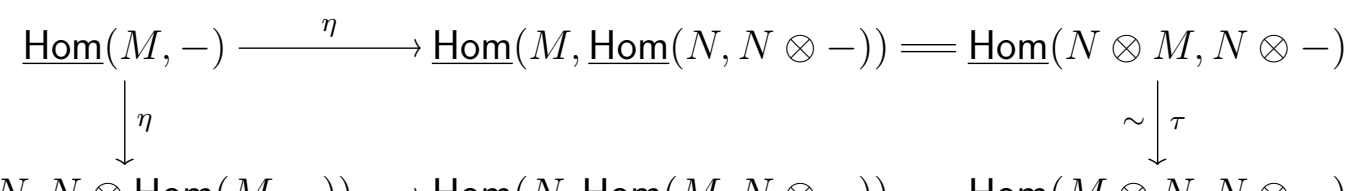

$\underline{\operatorname{Hom}}(N, N \otimes \underline{\operatorname{Hom}}(M,-)) \longrightarrow \underline{\operatorname{Hom}}(N, \underline{\operatorname{Hom}}(M, N \otimes-))=\underline{\operatorname{Hom}}(M \otimes N, N \otimes-)$

is commutative for $M, N \in \mathfrak{M}$. This is a particular case of Lemma 5.11 below.

LEMMA 5.11 - Let $\mathcal{C}$ be a category, $f_{1}$ and $f_{2}$ two endofunctors of $\mathcal{C}$, and $\tau: f_{1} \circ f_{2} \longrightarrow f_{2} \circ f_{1}$ a natural transformation. Assume that $f_{1}$ and $f_{2}$ admit right adjoints $g_{1}$ and $g_{2}$. Define a natural transformation $\sigma: f_{2} \circ g_{1} \longrightarrow g_{1} \circ f_{2}$ by the composition of

$$
f_{2} \circ g_{1} \stackrel{\eta}{\longrightarrow} g_{1} \circ f_{1} \circ f_{2} \circ g_{1} \stackrel{\tau}{\longrightarrow} g_{1} \circ f_{2} \circ f_{1} \circ g_{1} \stackrel{\delta}{\longrightarrow} g_{1} \circ f_{2} .
$$

Also, denote by $\tau^{\prime}: g_{1} \circ g_{2} \longrightarrow g_{2} \circ g_{1}$ the natural transformation deduced from $\tau$ by adjunction. Then, the following square

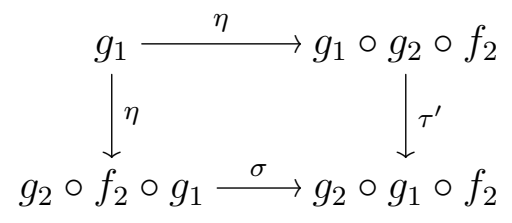

commutes. 
Proof. Indeed, $\sigma$ can also be defined as the composition of

$$
f_{2} \circ g_{1} \stackrel{\eta}{\longrightarrow} f_{2} \circ g_{1} \circ g_{2} \circ f_{2} \stackrel{\tau^{\prime}}{\longrightarrow} f_{2} \circ g_{2} \circ g_{1} \circ f_{1} \stackrel{\delta}{\longrightarrow} g_{1} \circ f_{1} .
$$

The claim thus follows from the commutativity of the following diagram

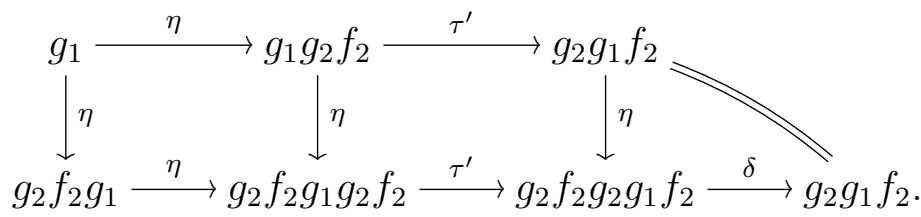

(The commutativity of the squares is obvious; the commutativity of the triangle follows from the definition of an adjunction.)

Remark 5.12 - Proposition 5.10, which implies in particular that the natural transformations

$$
\boldsymbol{\Lambda}\left(\lambda_{\mathbf{E}}\right), \lambda_{\mathbf{\Lambda}(\mathbf{E})}: \mathbf{\Lambda}(\mathbf{E}) \longrightarrow \boldsymbol{\Lambda} \circ \boldsymbol{\Lambda}(\mathbf{E})
$$

are not equal, is in contrast with [2, Lemme 4.3.62]. (Of course, in loc. cit., $\boldsymbol{\Lambda}$ and $\lambda$ denote different, albeit related, functor and natural transformation.)

To remedy the issue in the previous remark, we need the following construction.

Construction 5.13 - Let $\mathbf{E}$ be a commutative $T$-spectrum. There is a natural action of $\Sigma_{n}$ on $\boldsymbol{\Lambda}^{\circ n}(\mathbf{E})$ which is obtained via the identification

$$
\Lambda^{\circ n}(\mathbf{E})=\mathrm{s}_{-}^{\circ n} \underline{\operatorname{Hom}}(\overbrace{T \otimes \cdots \otimes T}^{n \text { times }}, \mathbf{E})
$$

from the natural action of $\Sigma_{n}$ on $T^{\otimes n}$. We set

$$
\widetilde{\Lambda}^{n}(\mathbf{E})=\Lambda^{\circ n}(\mathbf{E}) / \Sigma_{n} .
$$

By construction, we have natural transformations

$$
\widetilde{\lambda}^{n}: \mathrm{id} \longrightarrow \widetilde{\Lambda}^{n} \quad \text { and } \quad \mu^{m, n}: \widetilde{\Lambda}^{m} \circ \widetilde{\Lambda}^{n} \longrightarrow \widetilde{\Lambda}^{m+n} .
$$

COROLlARY $\mathbf{5 . 1 4}$ - For a commutative T-spectrum E, the square

$$
\begin{array}{r}
\widetilde{\boldsymbol{\Lambda}}^{m}(\mathbf{E}) \stackrel{\tilde{\boldsymbol{\Lambda}}^{m}\left(\tilde{\lambda}_{\mathbf{E}}^{n}\right)}{\longrightarrow} \widetilde{\boldsymbol{\Lambda}}^{m} \circ \widetilde{\boldsymbol{\Lambda}}^{n}(\mathbf{E}) \\
\widetilde{\boldsymbol{\Lambda}}^{n} \circ{\widetilde{\lambda^{\prime}}}^{m} \widetilde{\boldsymbol{\Lambda}}^{m}(\mathbf{E}) \stackrel{\mu_{\mathbf{E}}^{n, m}}{\longrightarrow} \widetilde{\boldsymbol{\Lambda}}^{m+n}(\mathbf{E})
\end{array}
$$

is commutative. We denote by $\widetilde{\lambda}_{\mathbf{E}}^{m, m+n}: \widetilde{\Lambda}^{m}(\mathbf{E}) \longrightarrow \widetilde{\Lambda}^{m+n}(\mathbf{E})$ the common value of the two possible compositions.

Proof. This is a direct consequence of Proposition 5.10 .

Construction $\mathbf{5 . 1 5}$ - Let $\mathbf{E}$ be a commutative $T$-spectrum. We define

$$
\widetilde{\Lambda}^{\infty}(\mathbf{E})=\operatorname{colim}_{m \in \mathbb{N}} \widetilde{\Lambda}^{m}(\mathbf{E})
$$

to be the colimit of the $\mathbb{N}$-system $\left\{\widetilde{\Lambda}^{m}(\mathbf{E})\right\}_{m \in \mathbb{N}}$ where the transition maps are given by $\widetilde{\lambda}_{\mathbf{E}}^{m, n}: \widetilde{\Lambda}^{m}(\mathbf{E}) \longrightarrow \widetilde{\Lambda}^{n}(\mathbf{E})$ for $m \leqslant n$. By construction, there is a natural transformation $\tilde{\lambda}_{\mathbf{E}}^{\infty}: \mathbf{E} \longrightarrow \widetilde{\Lambda}^{\infty}(\mathbf{E})$. 
THEOREM 5.16 - Let $\mathbf{E}$ be a levelwise fibrant commutative T-spectrum. Then $\widetilde{\Lambda}^{\infty}(\mathbf{E})$ is an $\Omega_{T}$-spectrum and $\widetilde{\lambda}_{\mathbf{E}}^{\infty}: \mathbf{E} \longrightarrow \widetilde{\Lambda}^{\infty}(\mathbf{E})$ is a stable equivalence.

Proof. For every $m \in \mathbb{N}$, the map $\mathbf{E} \longrightarrow \boldsymbol{\Lambda}^{\circ m}(\mathbf{E})$ is $\Sigma_{m}$-equivariant (with $\Sigma_{m}$ acting by identity on $\mathbf{E}$ ). On the other hand, by Theorem 5.8, this map is a stable equivalence. This implies that $\mathbf{E} \longrightarrow \widetilde{\Lambda}^{m}(\mathbf{E})$ is also a stable equivalence. As stable equivalences are preserved by transfinite compositions, we deduce that $\tilde{\lambda}_{\mathbf{E}}^{\infty}: \mathbf{E} \longrightarrow \widetilde{\Lambda}^{\infty}(\mathbf{E})$ is a stable equivalence.

We now prove that $\widetilde{\Lambda}^{\infty}(\mathbf{E})$ is an $\Omega_{T}$-spectrum. As $\underline{\operatorname{Rom}}(T,-)$ commutes with filtered colimits, it is enough to show that

$$
\lambda_{\widetilde{\mathbf{\Lambda}}^{\infty}(\mathbf{E})}: \widetilde{\boldsymbol{\Lambda}}^{\infty}(\mathbf{E}) \longrightarrow \boldsymbol{\Lambda} \circ \widetilde{\boldsymbol{\Lambda}}^{\infty}(\mathbf{E})
$$

is a levelwise weak equivalence. There are natural maps

$$
\mu_{\mathbf{E}}^{1, m}: \Lambda \circ \widetilde{\Lambda}^{m}(\mathbf{E}) \longrightarrow \widetilde{\Lambda}^{m+1}(\mathbf{E})
$$

which are levelwise weak equivalences since $\Sigma_{m+1}$ acts by identity on $T^{\otimes m+1}$ in $\operatorname{Ho}(\mathfrak{M})$. Passing to the colimit, we obtain a levelwise weak equivalence

$$
\mu_{\mathbf{E}}^{1, \infty}: \Lambda \circ \widetilde{\Lambda}^{\infty}(\mathbf{E}) \longrightarrow \widetilde{\Lambda}^{\infty}(\mathbf{E}) .
$$

Moreover, the composition of

$$
\widetilde{\Lambda}^{\infty}(\mathbf{E}) \longrightarrow \Lambda \circ \widetilde{\Lambda}^{\infty}(\mathbf{E}) \longrightarrow \widetilde{\Lambda}^{\infty}(\mathbf{E})
$$

is easily seen to be the identity (thanks to Corollary 5.14). This finishes the proof of the theorem.

Remark 5.17 - As far as I know, Theorem 5.16 has no analogue for symmetric $T$-spectra. However, in [5, §2.2.2], we were able to prove such an analogue in a particular situation and for a special sort of symmetric spectra, that were called $\boldsymbol{\Lambda}$-spectra. We refer the reader to [5, Définition 2.36] for the notion of $\boldsymbol{\Lambda}$-spectrum and to [5, Théorème 2.37] for the partial analogue of Theorem 5.16. Needless to say that Theorem 5.16 is much more satisfactory than [5, Théorème 2.37], although it requires a $\mathbb{Q}$-linear setting.

\section{The Betti monad, PART 1}

In this section, we revisit some of the constructions in [5, §2.2.3], taking advantage of the simplicity of the notion of commutative spectra (as opposed with that of symmetric spectra). These results, and in particular Theorem 6.20, are prerequisite for the results of Section 7, and in particular Theorem 7.16. However, Theorem 6.20 will not be explicitly used in Section 7 as we will be able to refer to [5] for the proofs. Nevertheless, we feel that Theorem 6.20 is of independent interest and clarifies the constructions in Section 7, so we decided to include it.

We remind the reader that the ring of coefficents $\Lambda$ is assumed to be a $\mathbb{Q}$-algebra. If not otherwise stated, our presheaves take values in the category of $\Lambda$-modules. We start by recalling [5, Définition 2.19]. For the notion of (co-)cubical object and its enriched variants, we refer the reader to [5, Définitions A.1, A.6 et A.12].

Definition 6.1 - For $n \in \mathbb{N}$, we denote by $\overline{\mathbb{D}}^{n}$ the closed unit polydisc of $\mathbb{C}^{n}$ considered as a complex pro-variety, i.e., we set

$$
\overline{\mathbb{D}}^{n}=\left\{\mathbb{D}(0, \rho)^{n}\right\}_{\rho>1}
$$


where $\mathbb{D}(0, \rho)=\{z \in \mathbb{C} ;|z|<\rho\}$. Varying $n \in \mathbb{N}$, one gets a $\Sigma$-enriched cocubical object $\overline{\mathbb{D}}$ in the category of complex pro-varieties in the following way.

(1) For $1 \leqslant i \leqslant n+1$ and $\epsilon \in\{0,1\}$, the face map $\mathrm{d}_{i, \epsilon}: \overline{\mathbb{D}}^{n} \hookrightarrow \overline{\mathbb{D}}^{n+1}$ is given by inserting the value of $\epsilon$ at the $i$-th coordinate, i.e.,

$$
\mathrm{d}_{i, \epsilon}\left(x_{1}, \ldots, x_{n}\right)=\left(x_{1}, \ldots, x_{i-1}, \epsilon, x_{i}, \ldots, x_{n}\right) .
$$

(2) For $1 \leqslant i \leqslant n$, the degeneracy map $\mathrm{p}_{i}: \overline{\mathbb{D}}^{n} \longrightarrow \overline{\mathbb{D}}^{n-1}$ is the projection parallel to the $i$-th factor, i.e.,

$$
\mathrm{p}_{i}\left(x_{1}, \ldots, x_{n}\right)=\left(x_{1}, \ldots, \widehat{x_{i}}, \ldots, x_{n}\right) .
$$

(3) For $1 \leqslant i \leqslant n-1$, the multiplication $\mathrm{m}_{i}: \overline{\mathbb{D}}^{n} \longrightarrow \overline{\mathbb{D}}^{n-1}$ is the multiplication of the $i$-th and $i+1$-th coordinates, i.e.,

$$
\mathrm{m}_{i}\left(x_{1}, \ldots, x_{n}\right)=\left(x_{1}, \ldots, x_{i-1}, x_{i} x_{i+1}, x_{i+2}, \ldots, x_{n}\right) .
$$

(4) For $n \in \mathbb{N}$ and $\sigma \in \Sigma_{n}$, the permutation map $\sigma: \overline{\mathbb{D}}^{n} \longrightarrow \overline{\mathbb{D}}^{n}$ is the permutation of coordinates, i.e.,

$$
\sigma\left(x_{1}, \ldots, x_{n}\right)=\left(x_{\sigma^{-1}(1)}, \ldots, x_{\sigma^{-1}(n)}\right) .
$$

Notation 6.2 - We denote by CpVar the category of smooth complex varieties. Given a presheaf $F$ on CpVar with values in a category admitting filtered colimits, we set

$$
F\left(\left(X_{i}\right)_{i}\right)=\operatorname{colim}_{i} F\left(X_{i}\right)
$$

whenever $\left(X_{i}\right)_{i}$ is a smooth complex pro-variety (i.e., a pro-object in CpVar).

Construction 6.3 - Given a complex of presheaves $K$ on CpVar, we set

$$
{ }^{\mathrm{a}} \mathrm{Sg}^{\mathbb{D}}(K)=\operatorname{Tot} \mathrm{A}(K(\overline{\mathbb{D}})) \text {. }
$$

In the formula above, Tot is the functor "total complex associated to a bicomplex" and $\mathrm{A}$ is as in [5, Définition A.20]. (Our sign convention will be as in [5, Remarque 2.21].) By construction, ${ }^{\mathrm{a}} \mathrm{Sg}^{\mathbb{D}}(K)$ is a complex of $\Lambda$-modules. We will also need a presheaf version of it, denoted by ${ }^{\mathrm{a}} \mathrm{Sg}^{\mathbb{D}}(K)$ and defined by

$$
{ }^{\mathrm{a}} \underline{\operatorname{Sg}}{ }^{\mathbb{D}}(K)=\operatorname{Tot} \mathrm{A}(\underline{\operatorname{hom}}(\overline{\mathbb{D}}, K)) .
$$

Clearly, we have ${ }^{\mathrm{a}} \mathrm{Sg} \mathbb{D}(K)=\Gamma\left(\mathrm{pt},{ }^{\mathrm{a}} \mathrm{Sg}^{\mathbb{D}}(K)\right)$ where $\Gamma(\mathrm{pt},-)$ is the "global sections" functor. There are also quasi-isomorphic variants of these constructions denoted by $\mathrm{Sg}^{\mathbb{D}}$ and ${ }^{\mathrm{n}} \mathrm{Sg} \mathbb{D}$ (and $\underline{\mathrm{Sg}^{\mathbb{D}}}$ and ${ }^{\mathrm{n}} \mathrm{Sg}^{\mathbb{D}}$ for the presheaf versions). We refer the reader to [5. Définition 2.20] for their definitions.

LEMMA 6.4 - The endofunctor ${ }^{\mathrm{a}} \mathrm{Sg}^{\mathbb{D}}$ of $\mathbf{C p l}(\mathbf{P S h}(\mathrm{CpVar} ; \Lambda))$ is naturally a monad.

Proof. The multiplication $\mathrm{m}:{ }^{\mathrm{a}} \mathrm{Sg}^{\mathbb{D}} \circ{ }^{\mathrm{a}} \mathrm{Sg}^{\mathbb{D}} \longrightarrow{ }^{\mathrm{a}} \mathrm{Sg}^{\mathbb{D}}$ is constructed in the same way as the morphism [5, (63)]. We leave the details to the reader.

We will endow the category CpVar with the classical (aka., usual, transcendental) topology which we denote by "cl". We warn the reader that in [3] and [5], we have used the symbol "usu" instead of "cl".

Remark 6.5 - The category $\mathbf{C p l}(\mathbf{P S h}(\mathrm{CpVar} ; \Lambda))$ admits three projective (resp. injective) model structures:

(1) the global model structure,

(2) the cl-local model structure, 
(3) the $\left(\mathbb{D}^{1}, \mathrm{cl}\right)$-local model structure.

We refer the reader to [3, §1] for the definitions and constructions of these model structures. We just remind the reader that the $\left(\mathbb{D}^{1}, \mathrm{cl}\right)$-local model structure is obtained as a Bousfield localisation of the cl-local model structure with respect to the maps $i_{0}: X \otimes \Lambda[n] \longrightarrow\left(\mathbb{D}^{1} \times X\right) \otimes \Lambda[n]$ where $n \in \mathbb{Z}, X \in \mathrm{CpVar}, \mathbb{D}^{1}$ is the unit open polydisc and $i_{0}$ is the zero section of $\mathbb{D}^{1}$. The homotopy category with respect to the $\left(\mathbb{D}^{1}, \mathrm{cl}\right)$-local model structure is denoted by $\operatorname{AnDA}^{\text {eff }}(\Lambda)$.

Recall the following result.

Proposition 6.6 - The "global sections" functor

$$
\Gamma(\mathrm{pt} ;-): \operatorname{Cpl}(\mathbf{P S h}(\mathrm{CpVar} ; \Lambda)) \longrightarrow \operatorname{Cpl}(\Lambda)
$$

is a right Quillen equivalence if the source is endowed with its projective $\left(\mathbb{D}^{1}, \mathrm{cl}\right)$-local model structure. In particular, we have an equivalence of categories

$$
\mathrm{R} \Gamma(\mathrm{pt} ;-): \operatorname{AnDA}^{\mathrm{eff}}(\Lambda) \stackrel{\sim}{\longrightarrow} \mathbf{D}(\Lambda) .
$$

Proof. This is a particular case of [3, Théorème 1.8].

THeOREM 6.7 - Let $K$ be a complex of presheaves on $\mathrm{CpVar}$. Then ${ }^{\mathrm{a}} \mathrm{Sg}^{\mathbb{D}}(K)$ is $\mathbb{D}^{1}$-local (considered as an object of $\mathbf{H o}_{\mathrm{cl}}(\mathbf{C p l}(\mathbf{P S h}(\mathrm{CpVar} ; \Lambda)))$ ). Moreover, the canonical morphism $K \longrightarrow{ }^{\mathrm{a}} \mathrm{Sg}^{\mathbb{D}}(K)$ is a local $\left(\mathbb{D}^{1}, \mathrm{cl}\right)$-equivalence. Said differently, ${ }^{\mathrm{a}} \mathrm{Sg} \mathbb{D}^{\mathbb{D}}$ is a $\mathbb{D}^{1}$-localisation functor.

Proof. This is [5, Théorème 2.23] since the complexes of presheaves $\underline{\operatorname{Sg}} \mathbb{D}(K)$ and ${ }^{\mathrm{a}} \mathrm{Sg}^{\mathbb{D}}(K)$ are naturally quasi-isomorphic.

COROLlary 6.8 - Let $K$ be a complex of presheaves on CpVar. Then, there is a natural isomorphism in $\mathbf{D}(\Lambda)$ :

$$
\mathrm{R} \Gamma(\mathrm{pt} ; K) \simeq{ }^{\mathrm{a}} \mathrm{Sg}^{\mathbb{D}}(K)
$$

where $\mathrm{R} \Gamma(\mathrm{pt} ;-)$ is the equivalence in Proposition 6.6.

Proof. This is [5, Corollary 2.26] modulo the quasi-isomorphism $\operatorname{Sg}^{\mathbb{D}}(K) \simeq{ }^{\mathrm{a}} \mathrm{Sg}^{\mathbb{D}}(K)$. Alternatively, it can be derived easily from Theorem 6.7.

To go further, we need the following construction.

Construction 6.9 - Let $K$ be a complex of presheaves on CpVar. For $m \in \mathbb{N}$, there is a natural transformation in $K$ :

$$
t_{m}: \underline{\operatorname{hom}}\left(\left(\overline{\mathbb{D}}^{m}, \partial \overline{\mathbb{D}}^{m}\right),{ }^{\mathrm{a}} \underline{\mathbb{D}}(K)\right) \longrightarrow{ }^{\mathrm{a}} \underline{\mathrm{Sg}} \mathbb{\mathbb { D }}(K)[-m]^{+}
$$

where the notations are as follows.

- $\left(\overline{\mathbb{D}}^{m}, \partial \overline{\mathbb{D}}^{m}\right)$ is the pro-pair where $\partial \overline{\mathbb{D}}^{m}$ is the divisor given by the union of the images of the faces $\mathrm{d}_{i, \epsilon}: \overline{\mathbb{D}}^{m-1} \hookrightarrow \overline{\mathbb{D}}^{m}$, for $1 \leqslant i \leqslant m$ and $\epsilon \in\{0,1\}$.

- The endofunctor hom $\left(\left(\overline{\mathbb{D}}^{m}, \partial \overline{\mathbb{D}}^{m}\right),-\right)$ is defined in [5, Définition 2.30].

- For a complex $A$, the complex $A[-m]^{+}$is obtained by translating the components of $A$ (so that $\left.\left(A[-m]^{+}\right)_{n}=A_{m+n}\right)$ without changing the sign of the differentials. Thus, for $m$ even, we have $A[-m]^{+}=A[-m]$. 
The morphism (24) is deduced from a morphism of bicomplexes

$$
\mathrm{A} \underline{\operatorname{hom}}\left(\left(\overline{\mathbb{D}}^{m}, \partial \overline{\mathbb{D}}^{m}\right), \underline{\operatorname{hom}}(\overline{\mathbb{D}}, K)\right) \longrightarrow \mathrm{A} \underline{\operatorname{hom}}(\overline{\mathbb{D}}, K)[-m]^{+},
$$

which, in degree $n \in \mathbb{N}$, is induced by the map of pro-pairs

$$
\overline{\mathbb{D}}^{n+m} \longrightarrow \overline{\mathbb{D}}^{n} \times\left(\overline{\mathbb{D}}^{m}, \partial \overline{\mathbb{D}}^{m}\right) .
$$

It follows immediately that (24) factors through the image of the projector

$$
\text { alt }_{m}=\frac{1}{m !} \sum_{\sigma \in \Sigma_{m}} \operatorname{sgn}(\sigma) \cdot \sigma
$$

acting on the source of (24). In particular, taking $2 m$ instead of $m$, we deduce a natural transformation

$$
l_{m}: \underline{\operatorname{hom}}\left(\left(\overline{\mathbb{D}}^{2}, \partial \overline{\mathbb{D}}^{2}\right)^{\wedge m},{ }^{\mathrm{a}} \underline{\operatorname{Sg}} \mathbb{D}(K)\right) / \Sigma_{m} \longrightarrow{ }^{\mathrm{a}} \mathrm{Sg}^{\mathbb{D}}(K)[2 m] .
$$

LEMMA 6.10 - Assume that $K$ is injectively fibrant (with respect to the global model structure). Then, (25) is a quasi-isomorphism of complexes of presheaves.

Proof. This follows immediately from [5, Lemme 2.33].

Recall that $T_{\mathrm{pt}}=\left(\mathbb{P}^{1, \text { an }}, \infty\right) \otimes \Lambda$. In what follows, the expression " $T_{\mathrm{pt}}$-spectrum" means " $T_{\mathrm{pt}}$-spectrum of complexes of presheaves on CpVar".

Remark 6.11 - The endofunctor ${ }^{\mathrm{a}} \mathrm{Sg}^{\mathbb{D}}$ extends to an endofunctor on the category of $T_{\mathrm{pt}}$-spectra. Given a $T_{\mathrm{pt}}$-spectrum $\mathbf{E}$, the $T_{\mathrm{pt}}$-spectrum ${ }^{\mathrm{a}}{ }_{\mathrm{Sg}} \mathbb{D}(\mathbf{E})$ is given in level $n$ by ${ }^{\text {a }} \underline{\underline{ }} \mathbb{D}\left(\mathbf{E}_{n}\right)$. Moreover, the adjoint of the assembly map at level $n$ is given by

$$
{ }^{\mathrm{a}} \underline{\operatorname{Sg}} \mathbb{D}\left(\mathbf{E}_{n}\right) \stackrel{\gamma_{n}^{\prime}}{\longrightarrow}{ }^{\mathrm{a}} \underline{\mathbb{D}} \mathbb{D}\left(\underline{\operatorname{hom}}\left(\left(\mathbb{P}^{1, \text { an }}, \infty\right), \mathbf{E}_{n+1}\right)\right) \stackrel{\tau}{\sim} \underline{\operatorname{hom}}\left(\left(\mathbb{P}^{1, \text { an }}, \infty\right),{ }^{\mathrm{a}} \underline{\operatorname{Sg}} \mathbb{D}\left(\mathbf{E}_{n+1}\right)\right) .
$$

If the $T_{\mathrm{pt}}$-spectrum $\mathbf{E}$ is commutative, so is the $T_{\mathrm{pt}}$-spectrum ${ }^{\mathrm{a}} \mathrm{Sg}^{\mathbb{D}}(\mathbf{E})$.

Remark 6.12 - Similarly, the functor hom $\left(\left(\overline{\mathbb{D}}^{2}, \partial \overline{\mathbb{D}}^{2}\right)^{\wedge m},-\right)$ extends to the category of $T_{\mathrm{pt}}$-spectra and preserves the commutative ones. Moreover, the natural transformation $l_{m}$ extends as well into a natural transformation

$$
l_{m}: \underline{\operatorname{hom}}\left(\left(\overline{\mathbb{D}}^{2}, \partial \overline{\mathbb{D}}^{2}\right)^{\wedge m}, \underline{{ }^{a}}{ }^{\mathbb{D}}(\mathbf{E})\right) / \Sigma_{m} \longrightarrow{ }^{\mathrm{a}} g^{\mathbb{D}}(\mathbf{E})[2 m]
$$

on the category of (commutative) $T_{\mathrm{pt}}$-spectra.

As in [5, Page 66], we fix an element $\alpha \in T_{\mathrm{pt}}\left(\overline{\mathbb{D}}^{2}, \partial \overline{\mathbb{D}}^{2}\right)$ whose class in homology freely generates the $\Lambda$-module $\mathrm{H}_{2}\left(\mathrm{Sg}^{\mathbb{D}}\left(T_{\mathrm{pt}}\right)\right)$. This choice induces a natural transformation

$$
\alpha^{*}: \underline{\operatorname{hom}}\left(\left(\mathbb{P}^{1, \text { an }}, \infty\right), K\right) \longrightarrow \underline{\operatorname{hom}}\left(\left(\overline{\mathbb{D}}^{2}, \partial \overline{\mathbb{D}}^{2}\right), K\right) .
$$

DEFINITION 6.13 - Let $\mathbf{E}$ be a commutative $T_{\mathrm{pt}}$-spectrum.

(a) We define a natural transformation

$$
\varsigma_{\mathbf{E}}^{m}: \widetilde{\boldsymbol{\Lambda}}^{m}\left({ }^{\mathrm{a}} \underline{\operatorname{Sg}}^{\mathbb{D}}(\mathbf{E})\right) \longrightarrow \mathrm{s}_{-}^{\circ m} \circ{ }^{\mathrm{a}} \underline{S g}^{\mathbb{D}}(\mathbf{E})[-2 m]
$$

by taking the composition of

$$
\begin{aligned}
& \mathrm{S}_{-}^{\circ m} \underline{\operatorname{hom}}\left(\left(\mathbb{P}^{1, \text { an }}, \infty\right)^{\wedge m},{ }^{\mathrm{a}} \underline{\operatorname{Sg}} \mathbb{D}(\mathbf{E})\right) / \Sigma_{m} \\
& \downarrow^{\left(\alpha^{\wedge m}\right)^{*}} \\
& \mathrm{~S}_{-}^{\circ m} \underline{\operatorname{hom}}\left(\left(\overline{\mathbb{D}}^{2}, \partial \overline{\mathbb{D}}^{2}\right)^{\wedge m},{ }^{\mathrm{a}} \underline{\operatorname{Sg}} \mathbb{\mathbb { D }}(\mathbf{E})\right) / \Sigma_{m} \stackrel{l_{m}}{\longrightarrow} \mathrm{S}_{-}^{\circ m} \circ{ }^{\mathrm{a}} \underline{\operatorname{Sg}} \mathbb{D}(\mathbf{E})[-2 m] .
\end{aligned}
$$


(b) We define a natural transformation

$$
\vartheta_{\mathbf{E}}:{ }^{\mathrm{a}} \underline{\underline{\operatorname{Sg}}}{ }^{\mathbb{D}}(\mathbf{E}) \longrightarrow \mathrm{S}_{-} \circ{ }^{\mathrm{a}} \underline{\mathrm{Sg}^{\mathbb{D}}}(\mathbf{E})[-2]
$$

by taking the composition of

$$
\begin{aligned}
& { }^{\mathrm{a}} \underline{\operatorname{Sg}} \mathbb{D}(\mathbf{E}) \stackrel{\lambda}{\longrightarrow} \mathrm{s}_{-} \underline{\operatorname{hom}}\left(\left(\mathbb{P}^{1, \text { an }}, \infty\right),{ }^{\mathrm{a}} \underline{ }{ }^{\mathbb{D}}(\mathbf{E})\right) \\
& \alpha^{*} \\
& \mathrm{~S}_{-} \underline{\operatorname{hom}}\left(\left(\overline{\mathbb{D}}^{2}, \partial \overline{\mathbb{D}}^{2}\right),{ }^{\mathrm{a}} \underline{\operatorname{Sg}} \mathbb{D}(\mathbf{E})\right) \stackrel{l_{1}}{\longrightarrow} \mathrm{S}_{-} \circ{ }^{\mathrm{a}} \underline{\operatorname{Sg}} \mathbb{\mathbb { D }}(\mathbf{E})[-2] \text {. }
\end{aligned}
$$

Thus, we have $\vartheta_{\mathbf{E}}=\varsigma_{\mathbf{E}}^{1} \circ \lambda_{\mathbf{E}}$.

Remark 6.14 - For $n \in \mathbb{N}$, the two maps

$$
\mathrm{s}_{-}^{\circ n}\left(\vartheta_{\mathbf{E}}\right), \vartheta_{\mathrm{S}_{-}^{\circ n}(\mathbf{E})}: \mathrm{s}_{-}^{\circ n} \circ{ }^{\mathrm{a}} \underline{\mathrm{Sg}^{\mathbb{D}}}(\mathbf{E}) \longrightarrow \mathrm{s}_{-}^{\circ n+1} \circ{ }^{\mathrm{a}} \underline{\operatorname{Sg}} \mathbb{D}(\mathbf{E})[-2]
$$

coincide. This is compatible with [5, Lemme 2.47] since, for a commutative $T_{\mathrm{pt}^{-}}$ spectrum $\mathbf{E}$, the action of $\Sigma_{n+1}$ on $\mathbf{s}_{-}^{\circ+1} \mathbf{E}$ is trivial. In particular, there is no ambiguity in defining a map

$$
\vartheta_{\mathbf{E}}^{m}:{ }^{\mathrm{a}} \underline{\operatorname{Sg}}^{\mathbb{D}}(\mathbf{E}) \longrightarrow \mathrm{s}_{-}^{\circ m} \circ{ }^{\mathrm{a}} \underline{\operatorname{Sg}}^{\mathbb{D}}(\mathbf{E})[-2 m]
$$

as an iteration of the natural transformation of Definition 6.13(b).

LEMMA 6.15 - For all $m \in \mathbb{N}$, we have a commutative triangle:

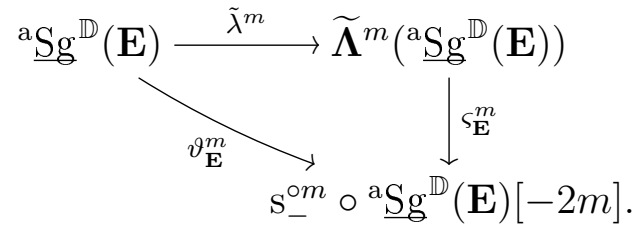

For all $0 \leqslant m \leqslant n$, we have a commutative square:

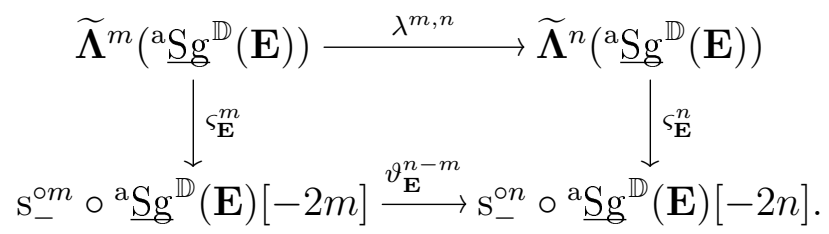

Proof. We leave this to the reader.

Proposition 6.16 - Let $\mathbf{E}$ be a commutative $T_{\mathrm{pt}^{-}}$-spectrum. Then $\vartheta_{\mathbf{E}}^{m}$ is a stable $\left(\mathbb{D}^{1}, \mathrm{cl}\right)$-local equivalence. If $\mathbf{E}$ is levelwise projectively $\left(\mathbb{D}^{1}, \mathrm{cl}\right)$-fibrant, then $\varsigma_{\mathbf{E}}^{m}$ is a levelwise quasi-isomorphism.

Proof. We repeat the proof of [5, Proposition 2.43] using Theorem 5.8 instead of [5. Proposition 2.38]. (Note that in [5, Proposition 2.43] we only stated a weaker conclusion about $\varsigma_{\mathbf{E}}$, namely that the latter is a levelwise $\left(\mathbb{D}^{1}, \mathrm{cl}\right)$-local equivalence. Nevertheless, in loc. cit., the stronger conclusion holds as well.)

As in the proof of [5, Proposition 2.43], we may assume that $\mathbf{E}$ is levelwise injectively $\left(\mathbb{D}^{1}, \mathrm{cl}\right)$-fibrant. It follows, as in loc. cit., that $\varsigma_{\mathbf{E}}^{m}$ is a levelwise $\left(\mathbb{D}^{1}, \mathrm{cl}\right)$-local equivalence. Since the source and the target of $\varsigma_{\mathbf{E}}^{m}$ are projectively $\left(\mathbb{D}^{1}, \mathrm{cl}\right)$-fibrant (by [5, Lemme 2.28]), it follows that $\varsigma_{\mathbf{E}}^{m}$ is even a levelwise quasi-isomorphism. 
Now, using the commutative triangle in Lemma 6.15, it remains to show that

$$
\widetilde{\lambda}^{m}:{ }^{\mathrm{a}} \underline{\operatorname{Sg}} \mathbb{D}(\mathbf{E}) \longrightarrow \widetilde{\Lambda}^{m}\left({ }^{\mathrm{a}} \underline{\operatorname{Sg}}^{\mathbb{D}}(\mathbf{E})\right)
$$

is a stable $\left(\mathbb{D}^{1}, \mathrm{cl}\right)$-local equivalence. This follows from Theorem 5.8 since, by 5 , Lemme 2.28], ${ }^{\mathrm{a}} \mathrm{Sg}^{\mathbb{D}}(\mathbf{E})$ is levelwise projectively $\left(\mathbb{D}^{1}, \mathrm{cl}\right)$-fibrant.

DeFinition 6.17 - Let $\mathbf{E}$ be a commutative $T_{\mathrm{pt}}$-spectrum. We set

$$
{ }^{\mathrm{a}} \operatorname{Sing} \mathbb{\mathbb { D }}(\mathbf{E})=\underset{n \in \mathbb{N}}{\operatorname{colim}} \mathrm{s}_{-}^{\circ n} \circ{ }^{\mathrm{a}} \underline{\underline{\operatorname{Sg}}} \mathbb{D}(\mathbf{E})[-2 n],
$$

the colimit of the $\mathbb{N}$-system

$$
\begin{aligned}
& { }^{\mathrm{a}} \underline{\mathrm{Sg}} \mathbb{\mathbb { D }}(\mathbf{E}) \stackrel{\vartheta_{\mathbf{E}}}{\longrightarrow} \mathrm{s}_{-} \circ{ }^{\mathrm{a}} \underline{\mathrm{Sg}} \mathbb{\mathbb { D }}(\mathbf{E})[-2] \stackrel{\vartheta_{\mathbf{E}}}{\longrightarrow} \cdots \stackrel{\vartheta_{\mathbf{E}}}{\longrightarrow} \mathrm{s}_{-}^{\circ n} \circ{ }^{\mathrm{a}} \underline{\mathrm{Sg}} \mathbb{\mathbb { D }}(\mathbf{E})[-2 n] \\
& \stackrel{\vartheta_{\mathbf{E}}}{\longrightarrow} \mathrm{s}_{-}^{\circ n+1} \circ{ }^{\mathrm{a}} \underline{\mathrm{Sg}} \mathbb{D}(\mathbf{E})[-2 n-2] \stackrel{\vartheta_{\mathbf{E}}}{\longrightarrow} \cdots .
\end{aligned}
$$

Remark 6.18 - The endofunctor ${ }^{\mathrm{a}}$ Sing ${ }^{\mathbb{D}}$ coincides with the three endofunctors ${ }^{\mathrm{a}} \operatorname{Sing}{ }^{\mathbb{D}, \infty},{ }^{\mathrm{a}} \operatorname{Sing}{ }^{\mathbb{D}, \infty}$ and ${ }^{\mathrm{a}} \operatorname{Sing}{ }^{\mathbb{D}, \infty}, \infty$ of $[5]$ when restricted to commutative $T_{\mathrm{pt}^{-}}$ spectra. Only ${ }^{\text {a Sing }}{ }^{\mathbb{D}, \infty}$ was explicitly defined in [5]; see [5, Définition 2.45]. The other two appear in [5, Page 89]. This explains the missing " $\infty$ " in our notation. (We refer the reader to Remark 7.13 for further explanations in an analogous context.)

LEMмA 6.19 - The endofunctor ${ }^{\text {a Sing }}{ }^{\mathbb{D}}$ of the category

$$
\mathbf{S p t}_{T_{\mathrm{pt}}}^{\sharp}(\mathbf{C p l}(\mathbf{P S h}(\mathrm{CpVar} ; \Lambda)))
$$

is naturally a pseudo-monoidal monad.

Proof. The monad structure on ${ }^{\text {a }}$ Sing $^{\mathbb{D}}$ is induced from the monad structure on ${ }^{\text {a }} \mathrm{Sg}^{\mathbb{D}}$ (see Lemma 6.4). We refer the reader to Lemma 7.14 and the references used in its proof for further explanations in an analogous context.

The following is the main result of this section.

THEOREM 6.20 - Let $\mathbf{E}$ be a commutative $T_{\mathrm{pt}}$-spectrum. The canonical morphism $\mathbf{E} \longrightarrow{ }^{\mathrm{a}} \operatorname{Sing}^{\mathbb{D}}(\mathbf{E})$ is a stable $\left(\mathbb{D}^{1}, \mathrm{cl}\right)$-local equivalence and ${ }^{\mathrm{a}} \mathbf{S i n g}^{\mathbb{D}}(\mathbf{E})$ is an $\Omega$-spectrum which is levelwise $\mathbb{D}^{1}$-local. Said differently, ${ }^{\text {a Sing }}{ }^{\mathbb{D}}$ is a stable $\mathbb{D}^{1}$ localisation functor.

Proof. We repeat the proof of [5, Théorème 2.48] using Theorem 5.16 instead of [5, Théorème 2.37].

As in loc. cit., we know that the commutative $T_{\mathrm{pt}^{-} \text {-Spectrum }}{ }^{\mathrm{a}} \operatorname{Sing}^{\mathbb{D}}(\mathbf{E})$ is levelwise $\mathbb{D}^{1}$-local and that the morphism $\mathbf{E} \longrightarrow{ }^{\mathrm{a}} \operatorname{Sing}^{\mathbb{D}}(\mathbf{E})$ is a stable $\left(\mathbb{D}^{1}, \mathrm{cl}\right)$-local equivalence. (The latter property follows from Proposition 6.16.) Thus, it remains to show that ${ }^{\text {a }} \operatorname{Sing}^{\mathbb{D}}(\mathbf{E})$ is an $\Omega$-spectrum.

As in loc. cit., we may assume that $\mathbf{E}$ is levelwise projectively $\left(\mathbb{D}^{1}, \mathrm{cl}\right)$-fibrant. The same is then true for ${ }^{\mathrm{a}} \mathrm{Sg}^{\mathbb{D}}(\mathbf{E})$. Consider the morphism of $\mathbb{N}$-systems

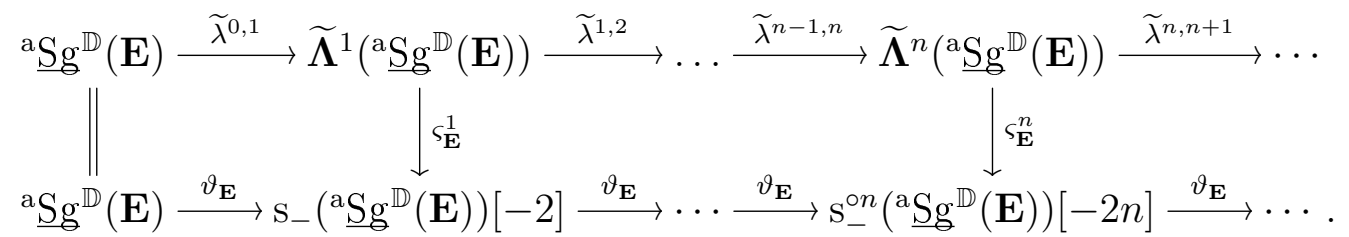


(The diagram commutes thanks to Lemma 6.15.) The vertical arrows are levelwise quasi-isomorphisms by Proposition 6.16. Taking the colimit, one gets a levelwise quasi-isomorphism

$$
\varsigma_{\mathbf{E}}^{\infty}: \widetilde{\Lambda}^{\infty}\left({ }^{\mathrm{a}} \underline{\operatorname{Sg}}^{\mathbb{D}}(\mathbf{E})\right) \longrightarrow{ }^{\mathrm{a}} \operatorname{Sing}^{\mathbb{D}}(\mathbf{E})
$$

By Theorem 5.16, the source of this morphism is an $\Omega$-spectrum. This finishes the proof.

For completeness, we recall the following.

Proposition 6.21 - The "global sections" functor

$$
\Gamma\left(\mathrm{pt} ; \operatorname{Ev}_{0}(-)\right): \mathbf{S p t}_{T_{\mathrm{pt}}}^{\sharp}(\mathbf{C p l}(\mathbf{P S h}(\mathrm{CpVar} ; \Lambda))) \longrightarrow \mathbf{C p l}(\Lambda)
$$

is a right Quillen equivalence if the source is endowed with its projective stable $\left(\mathbb{D}^{1}, \mathrm{cl}\right)$-local model structure. In particular, we have an equivalence of categories

$$
\mathrm{R} \Gamma\left(\mathrm{pt} ; \operatorname{REv}_{0}(-)\right): \operatorname{AnDA}(\Lambda) \stackrel{\sim}{\longrightarrow} \mathbf{D}(\Lambda)
$$

where $\operatorname{AnDA}(\Lambda)$ denotes the homotopy category with respect to the stable $\left(\mathbb{D}^{1}, \mathrm{cl}\right)$ local model structure.

Proof. This follows from Proposition 6.6 as explained in [3, Pages 238-239].

By a standard argument, we get the following corollary from Theorem 6.20.

Corollary 6.22 - Let $\mathbf{E}$ be a commutative $T_{\mathrm{pt}^{-}}$-spectrum. Then, there is a natural isomorphism in $\mathbf{D}(\Lambda)$ :

$$
\mathrm{R} \Gamma\left(\mathrm{pt} ; \operatorname{REv}_{0}(\mathbf{E})\right) \simeq \Gamma\left(\mathrm{pt} ; \operatorname{Ev}_{0}\left({ }^{\mathrm{a}} \operatorname{Sing}(\mathbf{E})\right)\right)
$$

where $\mathrm{R} \Gamma\left(\mathrm{pt} ; \mathrm{REv}_{0}(-)\right)$ is the equivalence in Proposition 6.21.

\section{The Betti MONAD, PART 2}

We fix a base field $k$ and a complex embedding $\sigma: k \hookrightarrow \mathbb{C}$. In this section, we will study "semi-algebraic" versions of some of the constructions in Section 6 .

Notation 7.1 - For $n \in \mathbb{N}$, we denote by $\overline{\mathbb{D}}_{\text {êt }}^{n}$ the pro- $k$-variety of étale neighbourhoods of $\overline{\mathbb{D}}^{n}$ in $\mathbb{A}_{k}^{n}$. More precisely, let $\mathscr{V}_{\text {êt }}\left(\overline{\mathbb{D}}^{n} / \mathbb{A}_{k}^{n}\right)$ be the category of pairs $(U, u)$ where $U$ is an étale $\mathbb{A}_{k}^{n}$-scheme and $u: \overline{\mathbb{D}}^{n} \longrightarrow U^{\text {an }}$ is a morphism of complex pro-varieties making the triangle

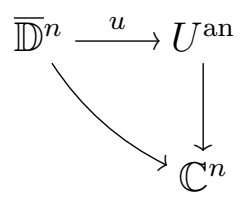

commutative. By [5, Proposition 2.58], the category $\mathscr{V}_{\text {êt }}\left(\overline{\mathbb{D}}^{n} / \mathbb{A}_{k}^{n}\right)$ is cofiltered and the functor

$$
\begin{aligned}
\overline{\mathbb{D}}_{\text {êt }}^{n}: \mathscr{V}_{\text {èt }}\left(\overline{\mathbb{D}}^{n} / \mathbb{A}_{k}^{n}\right) & \longrightarrow \mathrm{Sm} / k \\
(U, u) & \mapsto U
\end{aligned}
$$

defines a pro- $k$-scheme $\overline{\mathbb{D}}_{\text {êt }}^{n}$ which is pro-étale over $\mathbb{A}_{k}^{n}$. Also, it follows from [5, Proposition 2.58] that $\overline{\mathbb{D}}_{\text {êt }}^{n}$ is pro-affine (and more precisely, isomorphic to a proobject in the category of affine étale $\mathbb{A}_{k}^{n}$-schemes). Moreover, by [5, Proposition 2.102], $\mathcal{O}\left(\overline{\mathbb{D}}_{\text {êt }}^{n}\right)$ is the subalgebra of $\mathcal{O}\left(\overline{\mathbb{D}}^{n}\right)$ consisting of those holomorphic functions which are algebraic over the field of rational functions $\operatorname{Frac} \mathcal{O}\left(\mathbb{A}_{k}^{n}\right)$. 
Definition $7.2-A s$ in Definition 6.1 , one gets, by varying $n \in \mathbb{N}$, a $\Sigma$ enriched cocubical object $\overline{\mathbb{D}}_{\text {ét }}$ in the category of pro-k-varieties. The $\Sigma$-enriched cubical algebra $\mathcal{O}\left(\overline{\mathbb{D}}_{\text {êt }}\right)$ is naturally a $\Sigma$-enriched cubical subalgebra of $\mathcal{O}(\overline{\mathbb{D}})$ and this property determines the structural maps of $\overline{\mathbb{D}}_{\text {ét }}$ uniquely. (For this reason, we will not spell these out.)

Construction 7.3 - Given a complex of presheaves $K$ on $\mathrm{Sm} / k$, we set (by analogy with Construction 6.3

$$
{ }^{\mathrm{a}} \operatorname{Sg}_{\text {ét }}^{\mathbb{D}}(K)=\operatorname{Tot} \mathrm{A}\left(K\left(\overline{\mathbb{D}}_{\text {ét }}\right)\right) \text {. }
$$

By construction, ${ }^{\text {a }} \mathrm{Sg}_{\text {ét }} \mathbb{D}(K)$ is a complex of $\Lambda$-modules. We also need a presheaf version of it, denoted by ${ }^{\mathrm{a}} \underline{S g}_{\text {êt }}^{\mathbb{D}}(K)$ and defined by

$$
{ }^{\mathrm{a}} \underline{\operatorname{Sg}}_{\text {ét }}^{\mathbb{D}}(K)=\operatorname{Tot} \mathrm{A}\left(\underline{\text { hom }}\left(\overline{\mathbb{D}}_{\text {ét }}, K\right)\right) \text {. }
$$

Clearly, we have ${ }^{a} \operatorname{Sg}_{\text {êt }} \mathbb{D}(K)=\Gamma\left(k,{ }^{a} \operatorname{Sg}_{\text {êt }}^{\mathbb{D}}(K)\right)$ where $\Gamma(k,-)$ is the "global sections" functor. There are also quasi-isomorphic variants of these constructions denoted by $\mathrm{Sg}_{\text {ét }}^{\mathbb{D}}$ and ${ }^{\mathrm{n}} \mathrm{Sg}_{\text {ét }}^{\mathbb{D}}$ (and $\underline{S g}_{\text {ét }}^{\mathbb{D}}$ and ${ }^{\mathrm{n}} \underline{\mathrm{Sg}_{\text {ét }}} \mathbb{\mathbb { D }}$ for the presheaf versions); see [5, Remarque 2.60].

LEMMA 7.4 - The endofunctor ${ }^{\mathrm{a}} \mathrm{Sg}_{\text {ét }}^{\mathbb{D}}$ of $\operatorname{Cpl}(\mathbf{P S h}(\operatorname{Sm} / k ; \Lambda))$ is naturally a monad.

Proof. This is explained in [5, Page 84].

TheOREM 7.5 - Let $K$ be a complex of presheaves on $\mathrm{Sm} / k$. Then, there is a natural morphism

$$
{ }^{\mathrm{a}} \mathrm{Sg}_{\text {ét }} \mathbb{D}^{\mathbb{D}}(K) \longrightarrow{ }^{\mathrm{a}} \mathrm{Sg}^{\mathbb{D}}\left(A n^{*}(K)\right)
$$

which is a quasi-isomorphism. (Recall that $A n^{*}$ is the inverse image functor along the functor $A n: \mathrm{Sm} / k \longrightarrow$ CpVar given by $X \mapsto X^{\mathrm{an}}$.)

Proof. This is the combination of [5, Lemme 2.55] and [5, Théorème 2.61].

As in [5, §2.1.2], we define the Betti realisation functor

$$
\mathrm{Bti}_{\sigma}^{\text {eff, } *}: \mathbf{D A}^{\text {eff, ét }}(k ; \Lambda) \longrightarrow \mathbf{D}(\Lambda)
$$

to be the composition of

$$
\mathbf{D A}^{\text {eff, ét }}(k ; \Lambda) \stackrel{\mathrm{L} A n^{*}}{\longrightarrow} \mathbf{A n D A}^{\mathrm{eff}}(\Lambda) \stackrel{\mathrm{R} \Gamma(\mathrm{pt} ;-)}{\sim} \mathbf{D}(\Lambda),
$$

where the second functor is the equivalence in Proposition 6.6.

Corollary 7.6 - Let $K$ be a complex of presheaves on $\mathrm{Sm} / k$ considered as an object of $\mathbf{D A}^{\mathrm{eff}, \mathrm{e} t}(k ; \Lambda)$. Then, there is a canonical isomorphism in $\mathbf{D}(\Lambda)$ :

$$
\operatorname{Bti}_{\sigma}^{\text {eff, } *}(K) \simeq{ }^{\text {a }} \operatorname{Sg}_{\text {ét }}^{\mathbb{D}}(K) \text {. }
$$

Proof. This follows from Corollary 6.8 and Theorem 7.5, see [5, Corollaire 2.63]. 77].

The following is a "semi-algebraic" analogue of Construction 6.9; see also [5, Page

Construction 7.7 - Let $K$ be a complex of presheaves on $\mathrm{Sm} / k$. For $m \in \mathbb{N}$, there is a natural transformation in $K$ :

$$
t_{m}: \underline{\operatorname{hom}}\left(\left(\overline{\mathbb{D}}_{\text {ét }}^{m}, \partial \overline{\mathbb{D}}_{\text {êt }}^{m}\right), \underline{\operatorname{Sg}}_{\text {ét }}^{\mathbb{D}}(K)\right) \longrightarrow{ }^{\mathrm{a}} \underline{\operatorname{Sg}}_{\text {ét }}^{\mathbb{D}}(K)[-m]^{+}
$$


where the notations are as follows.

- $\left(\overline{\mathbb{D}}_{\text {êt }}^{m}, \partial \overline{\mathbb{D}}_{\text {êt }}^{m}\right)$ is the pro-pair where $\partial \overline{\mathbb{D}}_{\text {êt }}^{m}$ is the divisor given by the union of the images of the faces $\mathrm{d}_{i, \epsilon}: \overline{\mathbb{D}}_{\mathrm{et}}^{m-1} \hookrightarrow \overline{\mathbb{D}}_{\text {êt }}^{m}$, for $1 \leqslant i \leqslant m$ and $\epsilon \in\{0,1\}$.

- The endofunctor hom $\left(\left(\overline{\mathbb{D}}_{\mathrm{et}}^{m}, \partial \overline{\mathbb{D}}_{\mathrm{et}}^{m}\right),-\right)$ is defined in [5, Page 77].

As in the analytic case (see Construction 6.9), the morphism (27) factors through the image of the projector alt $m$. In particular, taking $2 m$ instead of $m$, we deduce a natural transformation

$$
l_{m}: \underline{\operatorname{hom}}\left(\left(\overline{\mathbb{D}}_{\text {ét }}^{2}, \partial \overline{\mathbb{D}}_{\text {ét }}^{2}\right)^{\wedge m},{ }^{\mathrm{a}} \mathrm{Sg}_{\text {ét }}^{\mathbb{D}}(K)\right) / \Sigma_{m} \longrightarrow{ }^{\mathrm{a}} \underline{S g}_{\text {ét }}^{\mathbb{D}}(K)[2 m] .
$$

Recall that $T_{k}=\left(\mathbb{P}_{k}^{1}, \infty\right) \otimes \Lambda$. In what follows, the expression " $T_{k}$-spectrum" means " $T_{k}$-spectrum of complexes of presheaves on $\mathrm{Sm} / k$ ".

Remark 7.8 - The endofunctor ${ }^{a} \underline{S g}_{\text {ét }}^{\mathbb{D}}$ extends to an endofunctor on the category of $T_{k}$-spectra exactly as in Remark 6.11. This extension preserves commutative $T_{k}$-spectra. Also, the natural transformation $l_{m}$ of Construction 7.7 extends to a natural transformation

$$
l_{m}: \underline{\operatorname{hom}}\left(\left(\overline{\mathbb{D}}_{\text {ét }}^{2}, \partial \overline{\mathbb{D}}_{\text {êt }}^{2}\right)^{\wedge m}, \underline{\operatorname{Sg}}_{\text {ét }}^{\mathbb{D}}(\mathbf{E})\right) / \Sigma_{m} \longrightarrow{ }^{\mathrm{a}} \underline{\operatorname{Sg}}_{\text {êt }}^{\mathbb{D}}(\mathbf{E})[2 m]
$$

on the category of (commutative) $T_{k}$-spectra.

We now assume that the element $\alpha \in T_{\mathrm{pt}}\left(\overline{\mathbb{D}}^{2}, \partial \overline{\mathbb{D}}^{2}\right)$, that was chosen in Section 6 . belongs to $T_{k}\left(\overline{\mathbb{D}}_{\text {êt }}^{2}, \partial \overline{\mathbb{D}}_{\text {êt }}^{2}\right)$. This is possible by Theorem 7.5. It follows that the class in homology of the element $\alpha \in T_{k}\left(\overline{\mathbb{D}}_{\text {êt }}^{2}, \partial \overline{\mathbb{D}}_{\text {êt }}^{2}\right)$ freely generates the $\Lambda$-module $\mathrm{H}_{2}\left(\mathrm{Sg}_{\text {ét }}^{\mathbb{D}}\left(T_{k}\right)\right)$. The element $\alpha$ induces a natural transformation

$$
\alpha^{*}: \underline{\operatorname{hom}}\left(\left(\mathbb{P}_{k}^{1}, \infty\right),-\right) \longrightarrow \underline{\operatorname{hom}}\left(\left(\overline{\mathbb{D}}_{\text {êt }}^{2}, \partial \overline{\mathbb{D}}_{\text {êt }}^{2}\right),-\right) .
$$

Below is the "semi-algebraic" analogue of Definition 6.13(b).

DEFINITION 7.9 - Let $\mathbf{E}$ be a commutative $T_{k}$-spectrum. We define a natural transformation

$$
\vartheta_{\mathbf{E}}:{ }^{\mathrm{a}} \underline{\mathrm{Sg}}_{\text {ét }}^{\mathbb{D}}(\mathbf{E}) \longrightarrow \mathrm{S}_{-} \circ{ }^{\mathrm{a}} \underline{\mathrm{Sg}}_{\text {ét }}^{\mathbb{D}}(\mathbf{E})[-2]
$$

by taking the composition of

$$
\begin{aligned}
& { }^{\mathrm{a}} \underline{\operatorname{Sg}}_{\text {ét }}^{\mathbb{D}}(\mathbf{E}) \stackrel{\lambda}{\longrightarrow} \mathrm{S}_{-} \underline{\operatorname{hom}}\left(\left(\mathbb{P}_{k}^{1}, \infty\right),{ }^{\mathrm{a}} \underline{\operatorname{Sg}}_{\text {ét }}^{\mathbb{D}}(\mathbf{E})\right) \\
& \downarrow \alpha^{*} \\
& \mathrm{~S}_{-} \underline{\mathrm{hom}}\left(\left(\overline{\mathbb{D}}_{\text {êt }}^{2}, \partial \overline{\overline{\mathbb{D}}}_{\text {êt }}^{2}\right),{ }^{\mathrm{a}} \underline{\mathrm{Sg}_{\text {êt }}} \mathbb{\mathbb { D }}_{(\mathbf{E}}(\mathbf{E})\right) \stackrel{l_{1}}{\longrightarrow} \mathrm{S}_{-} \circ{ }^{\mathrm{a}} \underline{\mathrm{Sg}}_{\text {êt }}^{\mathbb{D}}(\mathbf{E})[-2] .
\end{aligned}
$$

Remark 7.10 - As in Remark 6.14, for $n \in \mathbb{N}$, the two maps

$$
\mathrm{s}_{-}^{\circ n}\left(\vartheta_{\mathbf{E}}\right), \vartheta_{\mathrm{s}_{-}^{\circ n}(\mathbf{E})}: \mathrm{s}_{-}^{\circ n} \circ{ }^{\mathrm{a}} \underline{S g}_{\text {ét }} \mathbb{D}(\mathbf{E}) \longrightarrow \mathrm{s}_{-}^{\circ n+1} \circ{ }^{\mathrm{a}} \underline{\operatorname{Sg}}_{\text {ét }}^{\mathbb{D}}(\mathbf{E})[-2]
$$

coincide. In particular, there is no ambiguity in defining a map

$$
\vartheta_{\mathbf{E}}^{m}:{ }^{a} \underline{\operatorname{Sg}}_{\text {êt }}^{\mathbb{D}}(\mathbf{E}) \longrightarrow \mathrm{s}_{-}^{o m} \circ{ }^{a} \underline{S g}_{\text {ét }}^{\mathbb{D}}(\mathbf{E})[-2 m]
$$

as an iteration of the natural transformation of Definition 7.9,

Definition 7.11 - Let $\mathbf{E}$ be a commutative $T_{k}$-spectrum. We set

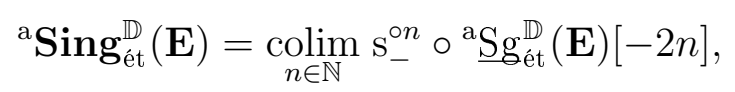

the colimit of the $\mathbb{N}$-system

$$
{ }^{\mathrm{a}} \underline{\mathrm{Sg}}_{\text {ét }}^{\mathbb{D}}(\mathbf{E}) \stackrel{\vartheta_{\mathbf{E}}}{\longrightarrow} \mathrm{S}_{-} \circ{ }^{\mathrm{a}} \underline{\mathrm{Sg}_{\text {ét }}} \mathbb{\mathbb { D }}(\mathbf{E})[-2] \stackrel{\vartheta_{\mathbf{E}}}{\longrightarrow} \cdots \stackrel{\vartheta_{\mathbf{E}}}{\longrightarrow} \mathrm{s}_{-}^{\circ n} \circ{ }^{\mathrm{a}} \underline{\mathrm{Sg}_{\text {ét }}}(\mathbf{E})[-2 n]
$$




$$
\stackrel{\vartheta_{\mathbf{E}}}{\longrightarrow} \mathrm{S}_{-}^{\circ n+1} \circ{ }^{\mathrm{a}} \underline{\mathrm{Sg}_{\text {ét }}^{\mathbb{D}}}(\mathbf{E})[-2 n-2] \stackrel{\vartheta_{\mathbf{E}}}{\longrightarrow} \cdots
$$

As in [5, §2.1.2] (and [3, Définition 2.1]), we define the Betti realisation functor

$$
\mathrm{Bti}_{\sigma}^{*}: \mathbf{D A}^{\text {ét }}(k ; \Lambda) \longrightarrow \mathbf{D}(\Lambda)
$$

to be the composition of

$$
\mathbf{D A}^{\text {ét }}(k ; \Lambda) \stackrel{\mathrm{L} A n^{*}}{\longrightarrow} \operatorname{AnDA}(\Lambda) \stackrel{\mathrm{R} \Gamma\left(\mathrm{pt} ; \mathrm{REv}_{0}(-)\right)}{\sim} \mathbf{D}(\Lambda),
$$

where the second functor is the equivalence in Proposition 6.21 .

CoRollary $\mathbf{7 . 1 2}$ - Let $\mathbf{E}$ be a commutative $T_{k}$-spectrum considered as an object of $\mathbf{D} \mathbf{A}^{\text {ét }}(k ; \Lambda)$. Then, there is a canonical isomorphism in $\mathbf{D}(\Lambda)$ :

$$
\operatorname{Bti}_{\sigma}^{*}(\mathbf{E}) \simeq \Gamma\left(k ; \operatorname{Ev}_{0}\left({ }^{a} \operatorname{Sing}_{\text {ét }}^{\mathbb{D}}(\mathbf{E})\right)\right) .
$$

Proof. This follows easily from Corollary 6.22 and Theorem 7.5, see also [5, Lemme 2.71].

Remark 7.13 - As in Remark 6.18, the endofunctor ${ }^{\text {a }}$ Sing $_{\text {ét }}^{\mathbb{D}}$ coincides with the three endofunctors ${ }^{\text {a }} \operatorname{Sing}_{\text {ét }}^{\mathbb{D}, \infty},{ }^{\text {a }} \operatorname{Sing}_{\text {ét }}{ }^{\mathbb{D},}$ and ${ }^{\mathrm{a}} \mathbf{S i n g}_{\text {ét }}^{\mathbb{D}, \infty, \infty}{ }^{\prime}$ of $[5]$ when restricted to commutative $T_{k}$-spectra. (See [5, Définition 2.65(ii)] for the definition of a ${ }^{\text {Singét }}{ }^{\mathbb{D},}$, [5. Page 86] for the definition of 'Sing ${ }_{\text {ét }}^{\mathbb{D}, \infty}$ ' and [5, Corollaire 2.81] for the definition of a ${ }^{\text {Sing }}$ ét $\infty, \infty^{\prime}$.) This explains the missing " $\infty$ " in our notation. As this plays an important role below, we explain the reason for which these three functors coincide on commutative $T_{k}$-spectra; this is based on the following two properties.

- First, the two natural transformations

$$
\vartheta_{\mathbf{E}}, \vartheta_{\mathbf{E}}^{\prime}:{ }^{\mathrm{a}} \underline{S g}_{\text {ét }}^{\mathbb{D}}(\mathbf{E}) \longrightarrow \mathrm{s}_{-} \circ{ }^{\mathrm{a}} \underline{\operatorname{Sg}}_{\text {ét }}^{\mathbb{D}}(\mathbf{E})[-2],
$$

where $\vartheta_{\mathbf{E}}^{\prime}$ is [5, (65)], coincide; this is [5, Proposition 2.79(b)] (which is valid, more generally, for $\mathbf{E}$ a symmetric $T_{k}$-spectrum).

- Second, as in Remark 7.10, the two natural transformations

$$
\mathrm{s}_{-}^{\circ n}\left(\vartheta_{\mathbf{E}}^{\prime}\right), \vartheta_{\mathrm{s}_{-}^{\circ n}(\mathbf{E})}^{\prime}: \mathrm{s}_{-}^{\circ n} \circ{ }^{\mathrm{a}} \underline{\operatorname{Sg}}_{\text {êt }}^{\mathbb{D}}(\mathbf{E}) \longrightarrow \mathrm{s}_{-}^{\circ n+1} \circ{ }^{\mathrm{a}} \underline{\operatorname{Sg}}_{\text {ét }}^{\mathbb{D}}(\mathbf{E})[-1]
$$

coincide. (This, contrary to the previous property, relies on the fact that $\mathbf{E}$ is a commutative $T_{k}$-spectrum.)

With these two properties in hand, one sees immediately that ${ }^{a} \mathbf{S i n g}_{\text {ét }}^{\mathbb{D}, \infty^{\prime}}(\mathbf{E})$, as defined in [5, Page 86], is equal to ${ }^{\mathrm{a}} \mathbf{S i n g}_{\text {ét }}^{\mathbb{D}},(\mathbf{E})$, as defined in [5, Définition 2.65(ii)]. Moreover, these two properties and [5. Lemme 2.80], imply that the maps

$$
\begin{gathered}
\vartheta_{\mathbf{E}}^{\prime}:{ }^{\mathrm{a}} \operatorname{Sing}_{\text {ét }}^{\mathbb{D}, \infty}(\mathbf{E}) \longrightarrow{ }^{\mathrm{a}} \operatorname{Sing}^{\mathbb{D}, \infty}\left(\mathrm{S}_{-} \mathbf{E}\right)[-2] \quad \text { and } \\
\vartheta_{\mathbf{E}}:{ }^{\mathrm{a}} \mathbf{S i n g}_{\text {ét }}^{\mathbb{D}, \infty^{\prime}}(\mathbf{E}) \longrightarrow \mathrm{S}_{-} \circ{ }^{\mathrm{a}} \mathbf{S i n g}_{\text {ét }}^{\mathbb{D}, \infty^{\prime}}(\mathbf{E})[-2]
\end{gathered}
$$

of [5, Corollary 2.81] are both isomorphisms of commutative $T_{k}$-spectra. This implies that the natural maps

$$
{ }^{\mathrm{a}} \operatorname{Sing}_{\text {êt }}^{\mathbb{D}, \infty}(\mathbf{E}) \longrightarrow{ }^{\mathrm{a}} \mathbf{S i n g}_{\text {êt }}^{\mathbb{D}, \infty, \infty}(\mathbf{E}) \text { and }{ }^{\mathrm{a}} \operatorname{Sing}_{\text {ét }}^{\mathbb{D}, \infty^{\prime}}(\mathbf{E}) \longrightarrow{ }^{\mathrm{a}} \mathbf{S i n g}_{\text {ét }}^{\mathbb{D}, \infty, \infty^{\prime}}(\mathbf{E})
$$

are isomorphisms. 
LEMMA 7.14 - The endofunctor ${ }^{\mathrm{a}} \mathbf{S i n g}_{\text {ét }}^{\mathbb{D}}$ of the category

$$
\operatorname{Spt}_{T_{k}}^{\sharp}(\mathbf{C p l}(\mathbf{P S h}(\operatorname{Sm} / k ; \Lambda)))
$$

is naturally a pseudo-monoidal monad.

Proof. The multiplication map

$$
\mathrm{m}:{ }^{\mathrm{a}} \operatorname{Sing}_{\text {ét }}^{\mathbb{D}} \circ{ }^{\mathrm{a}} \operatorname{Sing}_{\text {ét }}^{\mathbb{D}} \longrightarrow{ }^{\mathrm{D}} \operatorname{Sing}_{\text {ét }}^{\mathbb{D}}
$$

is [5, (67)] modulo the identifications of Remark 7.13. The pseudo-monoidal structure of a Sing êt ${ }^{\mathbb{D}}$ is the binatural transformation $\mathrm{m}$ at the end of [5, Page 92], modulo the identifications of Remark 7.13. The necessary properties that make ${ }^{\text {a }}$ Singét $_{\text {ento }}^{\mathbb{D}}$ a pseudo-monoidal monad follow from the corresponding properties of the pseudomonoidal monad ${ }^{a} \underline{S g}_{\text {ét }}^{\mathbb{D}}$.

Definition 7.15 - The monad ${ }^{\text {a }}$ Singét $_{\text {of Lemma } 7.14}^{\mathbb{D}}$ is called the Betti monad.

The terminology introduced in Definition 7.15 is justified by the following theorem.

THEOREM 7.16

(a) The endofunctor ${ }^{\text {a Sing }}{ }_{\text {ét }}^{\mathbb{D}}$ preserves projectively stably ( $\mathbb{A}^{1}$, ét)-fibrant commutative $T_{k}$-spectra. Moreover, it admits a right derived functor

$$
\mathrm{R}\left({ }^{\mathrm{a}} \mathbf{S i n g}_{\text {ét }}^{\mathbb{D}}\right): \mathbf{D A}^{\text {ét }}(k ; \Lambda) \longrightarrow \mathbf{D A}^{\text {ét }}(k ; \Lambda) \text {. }
$$

(b) Let $\mathbf{E}$ be a commutative $T_{k}$-spectrum and assume that $\mathbf{E}$ is projectively stably $\left(\mathbb{A}^{1}\right.$,ét)-fibrant. There exists a canonical isomorphism in $\mathbf{D A}^{\mathbf{e}^{\mathrm{e}}}(k ; \Lambda)$ :

$$
{ }^{\mathrm{a}} \operatorname{Sing}_{\text {ét }}^{\mathbb{D}}(\mathbf{E}) \simeq \operatorname{Bti}_{\sigma, *} \operatorname{Bti}_{\sigma}^{*}(\mathbf{E}) .
$$

(c) The isomorphisms in (b) define an invertible natural transformation

$$
\mathrm{R}\left({ }^{\mathrm{a}} \text { Sing }_{\text {ét }}^{\mathbb{D}}\right) \simeq \mathrm{Bti}_{\sigma, *} \mathrm{Bti}_{\sigma}^{*}
$$

of endofunctors of $\mathbf{D} \mathbf{A}^{\mathrm{e} t}(k ; \Lambda)$. Moreover, (30) is an isomorphism of pseudomonoidal monads.

Proof. Except the assertion that $(30)$ is a morphism of pseudo-monoidal monads, everything follows from [5, Théorème 2.67]. Indeed, a projectively stably ( $\mathbb{A}^{1}$,ét)fibrant commutative $T_{k}$-spectrum is also a projectively stably ( $\mathbb{A}^{1}$, ét)-fibrant symmetric $T_{k}$-spectrum. (In loc. cit., we work with the Nisnevich topology. However, the proof works as well with the étale topology, since, with $\Lambda$ being a $\mathbb{Q}$-algebra, filtered colimits preserve projectively ét-fibrant complexes of presheaves.)

The assertion that (30) is a morphism of pseudo-monoidal monads follows from [5, Théorème 2.82] and [5, Théorème 2.87], and the identifications in Remark 7.13.

\section{The motivic homotopy Hopf Algebra}

In this section, we put the results of Sections 3 and 7 together to construct the motivic homotopy Hopf algebra. We first check that the conditions in Setting 3.1 are satisfied.

Proposition 8.1 - Conditions (a)-(c) in Setting 3.1 are satisfied if we take:

- $\mathfrak{E}=\operatorname{Cpl}(\Lambda)$ with its projective model structure (making it a monoidal model category); 
- $\mathfrak{M}=\mathbf{S p t}_{T_{k}}^{\sharp}(\mathbf{C p l}(\mathbf{P S h}(\operatorname{Sm} / k ; \Lambda)))$ with its stable $\left(\mathbb{A}^{1}\right.$, ét $)$-local model structure of Definition 4.25 (making it a monoidal model category);

- $c=\operatorname{Sus}_{T_{k}, \sharp}^{0} \circ(-)_{\mathrm{cst}}$ (where $(-)_{\mathrm{cst}}$ is the "constant presheaf" functor) and $\Gamma=\Gamma\left(k ; \operatorname{Ev}_{0}(-)\right)$;

- $\mathcal{S}={ }^{\text {a Singét }} \mathbb{E}_{\text {dith }}^{\mathbb{D}}$ its structure of a monoidal monad (see Lemma 7.14).

Proof. By Theorem 7.16(a), the endofunctor ${ }^{\mathrm{a}} \mathbf{S i n g}_{\text {ét }}^{\mathbb{D}}$ preserves projectively stably ( $\mathbb{A}^{1}$, ét)-fibrant commutative $T_{k^{-}}$-spectra. Also, it clearly preserves levelwise quasiisomorphisms (and, in particular, stable $\left(\mathbb{A}^{1}\right.$, ét)-local equivalences between projectively stably $\left(\mathbb{A}^{1}\right.$, ét)-fibrant commutative $T_{k}$-spectra). Thus, condition (a) of Setting 3.1 is satisfied.

To verify condition (b) of Setting 3.1, we use the isomorphism (30) of Theorem 7.16(c). We thus need to show that the composition

$$
\mathrm{R} \Gamma\left(k ; \mathrm{REv}_{0}(-)\right) \circ \mathrm{Bti}_{\sigma *} \mathrm{Bti}_{\sigma}^{*}
$$

is monoidal. This would follows if we could show that the composition

$$
\mathrm{R} \Gamma\left(k ; \operatorname{REv}_{0}(-)\right) \circ \mathrm{Bti}_{\sigma *}
$$

is isomorphic to the identity functor since $\mathrm{Bti}_{\sigma}^{*}$ is monoidal. Thus, by adjunction, we are left to show that

$$
\operatorname{Bti}_{\sigma}^{*} \circ \operatorname{LSus}_{T_{k}, \sharp}^{0} \circ(-)_{\mathrm{cst}}
$$

is isomorphic to the identity functor, which is true by [5, Lemme 2.5].

Finally, condition (c) of Setting 3.1 follows from the fact that $\mathrm{Bti}_{\sigma}^{*}$ is monoidal and the fact that the binatural morphism

$$
\operatorname{Bti}_{\sigma, *}(A) \otimes M \longrightarrow \operatorname{Bti}_{\sigma, *}\left(A \otimes \operatorname{Bti}_{\sigma}^{*}(M)\right)
$$

is invertible for all $A \in \mathbf{D}(\Lambda)$ and $M \in \mathbf{D A}^{\text {ét }}(k ; \Lambda)$ by [5, Proposition 2.7(b)].

Remark 8.2 - With the notations as in Proposition 8.1, the monoidal functor $\mathrm{R} \Gamma \circ \mathrm{RS}$ of Setting 3.1(b) is nothing but the Betti realisation functor Bti* ${ }_{\sigma}^{*}$. (This follows from the proof of Proposition 8.1.) Said differently, one can recover the Betti realisation functor from the Betti monad using the formula

$$
\mathrm{Bti}_{\sigma}^{*}=\mathrm{R} \Gamma\left(k ; \operatorname{REv}_{0}\left(\mathrm{R}\left({ }^{\mathrm{a}} \mathbf{S i n g}_{\text {êt }}^{\mathbb{D}}\right)(-)\right)\right) .
$$

But we already know this formula: see Corollary 7.12 which also states that it is not necessary to derive ${ }^{\text {a }}$ Singét $_{\text {ét }}^{\mathbb{D}}$ in the above formula.

We now come to the main theorem of this paper. Let's denote by

$$
\mathbf{S}=\operatorname{Sus}_{T_{k}, \sharp}^{0}\left(\Lambda_{\mathrm{cst}}\right)
$$

the unit of the monoidal category $\operatorname{Spt}_{T_{k}}^{\sharp}(\mathbf{C p l}(\mathbf{P S h}(\operatorname{Sm} / k ; \Lambda)))$. Below, we use notation from Construction 3.9.

THEOREM $8.3-$ Let $\mathbf{S} \longrightarrow \mathbf{A}$ be a stable $\left(\mathbb{A}^{1}\right.$,ét)-local equivalence of commutative algebras in the category of commutative $T_{k}$-spectra such that $\mathbf{A}$ is projectively $\left(\mathbb{A}^{1}\right.$, ét)-fibrant.

(A) The cosimplicial algebra

$$
\underline{\mathcal{H}}_{\text {mot }}(k, \sigma ; \Lambda)=\Gamma\left(k ; \operatorname{Ev}_{0}\left(\widehat{\operatorname{Sing}}_{\text {ét }}^{\mathbb{D}}(\mathbf{A})\right)\right)
$$

is a homotopy Hopf algebra in $\operatorname{Cpl}(\Lambda)$. 
(B) Let $\mathbf{E}$ be an $\mathbf{A}$-module which is projectively ( $\mathbb{A}^{1}$,ét)-fibrant (as a commutative $T_{k}$-spectrum). Then, the $\underline{\mathcal{H}}_{\text {mot }}(k, \sigma ; \Lambda)$-module

$$
\underline{\mathrm{Bti}}_{\sigma}^{*}(\mathbf{E})=\Gamma\left(k ; \mathrm{Ev}_{0}\left({ }^{\mathrm{a}} \widehat{\mathbf{S i n g}_{\text {ét }}}(\mathbf{E})\right)\right)
$$

is a homotopy comodule over $\underline{\mathcal{H}}_{\text {mot }}(k, \sigma ; \Lambda)$.

(C) There is triangulated monoidal functor

$$
\underline{\operatorname{Bti}}_{\sigma}^{*}: \mathbf{D A}^{\text {ét }}(k ; \Lambda) \longrightarrow \operatorname{hocoMod}\left(\underline{\mathcal{H}}_{\text {mot }}(k, \sigma ; \Lambda)\right)
$$

which makes the triangle

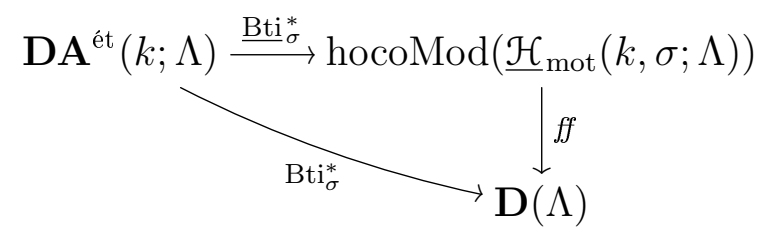

commutative up to a natural isomorphism.

Proof. Thanks to Proposition 8.1, the theorem follows, as a particular case, from Theorems 3.10 and 3.11. (The technical assumptions needed for Theorem 3.11 are satisfied by Lemma 4.27.)

For Theorem 8.3 to be applicable, we need to show that an algebra $\mathbf{A}$, as in the statement above, exists. To do so, we recall some notation.

Notation 8.4 - For a smooth $k$-variety $X, \Lambda_{\mathrm{tr}}(X)$ denotes the presheaf with transfers on $\mathrm{Sm} / k$ represented by $X$, i.e., given by

$$
\Lambda_{\mathrm{tr}}(X)(U)=\operatorname{Cor}(U, X) \otimes \Lambda \quad \text { for } \quad U \in \mathrm{Sm} / k,
$$

where $\operatorname{Cor}(U, X)$ is the group of finite correspondences from $U$ to $X$.

Notation 8.5 — We denote by

$$
\mathbf{S}^{\mathrm{tr}}=\operatorname{Sus}_{T_{k}^{\mathrm{tr}}, \sharp}^{0}\left(\Lambda_{\mathrm{cst}}\right)
$$

the analogue with transfers of $\mathbf{S}$. More precisely, after forgetting transfers, $\mathbf{S}^{\text {tr }}$ is the commutative $T_{k}$-spectrum given in level $n$ by

$$
\mathbf{S}_{n}^{\operatorname{tr}}=\Lambda_{\mathrm{tr}}\left(\left(\mathbb{P}_{k}^{1}, \infty\right)^{\wedge n}\right) / \Sigma_{n}
$$

(Recall that $\mathbf{S}_{n}=\left(\Lambda \otimes\left(\mathbb{P}_{k}^{1}, \infty\right)^{\wedge n}\right) / \Sigma_{n}$.) There is an obvious morphism of commutative $T_{k}$-spectra $\mathbf{S} \longrightarrow \mathbf{S}^{\text {tr }}$ which is easily seen to be a morphism of commutative algebras.

Notation 8.6 - As in Definitions 6.1 and 7.2 , there is a $\Sigma$-enriched cocubical $k$ variety $\mathbb{A}_{k}$ given in degree $n \in \mathbb{N}$ by $\mathbb{A}_{k}^{n}$. As in Constructions 6.3 and 7.3 , this cocubical $k$-variety can be used to construct an endofunctor ${ }^{a} \operatorname{Sg}^{\mathbb{A}}$ of $\mathbf{C p l}(\mathbf{P S h}(\operatorname{Sm} / k ; \Lambda))$. Of course, ${ }^{a} \mathrm{Sg}^{\mathbb{A}}$ is simply a cubical version of the well known "Suslin-Voevodsky complex".

We have the following result which relies on a number of results of Voevodsky and Friedlander-Voevodsky in [17].

Proposition 8.7 - The natural morphism $\mathbf{S} \longrightarrow \mathbf{S}^{\text {tr }}$ is a levelwise ( $\mathbb{A}^{1}$, ét)local equivalence and the commutative $T_{k}$-spectrum ${ }^{\mathrm{a}} \mathrm{Sg}^{\mathbb{A}}\left(\mathbf{S}^{\mathrm{tr}}\right)$ is projectively stably $\left(\mathbb{A}^{1}\right.$, ét)-fibrant. 
Proof. The first assertion follows form [5, Théorème B.1] as in the proof of [5, Corollaire 2.120]. (We may use [5, Théorème B.1] instead of [5, Corollaire 2.120] since $\Lambda$ is a $\mathbb{Q}$-algebra; this gives us the more precise statement that $\mathbf{S} \longrightarrow \mathbf{S}^{\text {tr }}$ is a levelwise $\left(\mathbb{A}^{1}\right.$, ét)-local equivalence instead of stable one.) The second assertion is a consequence of the second assertion in [5, Corollaire 2.120] and the fact that $\Sigma_{n}$ acts by identity on $\Lambda_{\mathrm{tr}}\left(\left(\mathbb{P}_{k}^{1}, \infty\right)^{\wedge n}\right)$ in $\mathbf{D} A^{\text {eff, ét }}(k ; \Lambda)$. (This point was discussed in Remark 4.26 .)

Remark 8.8 - Since the commutative $T_{k}$-spectrum ${ }^{\mathrm{a}}{ }^{\mathrm{Sg}}{ }^{\mathbb{A}}\left(\mathbf{S}^{\mathrm{tr}}\right)$ is a commutative algebra, Proposition 8.7 implies that we may take $\mathbf{A}={ }^{\mathrm{a}} \underline{S g}^{\mathbb{A}}\left(\mathbf{S}^{\operatorname{tr}}\right)$ in the statement of Theorem 8.3 to get the homotopy Hopf algebra

$$
\Gamma\left(k ; \operatorname{Ev}_{0}\left({ }^{a} \widehat{\operatorname{Sing}}_{\text {ét }}^{\mathbb{D}}\left({ }^{\mathrm{a}} \underline{\operatorname{Sg}}^{\mathbb{A}}\left(\mathbf{S}^{\mathrm{tr}}\right)\right)\right)\right) .
$$

On the other hand, by [5, 2.122], the natural transformation

$$
{ }^{\mathrm{a}} \underline{\operatorname{Sg}}_{\text {ét }}^{\mathbb{D}}(K) \longrightarrow{ }^{\mathrm{a}} \underline{\operatorname{Sg}}_{\text {ét }}^{\mathbb{D}}\left({ }^{\mathrm{a}} \underline{\operatorname{Sg}}^{\mathbb{A}}(K)\right)
$$

is a quasi-isomorphism for every complex of presheaves $K$ on $\mathrm{Sm} / k$. This implies that the natural morphism of commutative cosimplicial algebras

$$
\Gamma\left(k ; \mathrm{Ev}_{0}\left({ }^{\mathrm{a}} \widehat{\mathbf{S i n g}_{\text {ét }}}\left(\mathbf{S}^{\operatorname{tr}}\right)\right)\right) \longrightarrow \Gamma\left(k ; \operatorname{Ev}_{0}\left({ }^{a} \widehat{\operatorname{Sing}}_{\text {ét }}^{\mathbb{D}}\left({ }^{\mathrm{a}}{ }^{\mathrm{Sg}}{ }^{\mathbb{A}}\left(\mathbf{S}^{\mathrm{tr}}\right)\right)\right)\right)
$$

is a quasi-isomorphism. This motivates the following definition.

DEFinition 8.9 - The motivic homotopy Hopf algebra (of the field $k$ endowed with the complex embedding $\sigma$ ) is

$$
\underline{\mathcal{H}}_{\text {mot }}(k, \sigma ; \Lambda)=\Gamma\left(k ; \operatorname{Ev}_{0}\left(\widehat{\operatorname{Sing}}_{\text {ét }}^{\mathbb{D}}\left(\mathbf{S}^{\text {tr }}\right)\right)\right) .
$$

There is a triangulated monoidal functor

$$
\underline{\operatorname{Bti}}_{\sigma}^{*}: \mathbf{D A}^{\text {ét }}(k ; \Lambda) \longrightarrow \operatorname{hocoMod}\left(\underline{\mathcal{H}}_{\text {mot }}(k, \sigma ; \Lambda)\right)
$$

which factors the Betti realisation.

Remark 8.10 - By Theorem 3.12, the Hopf algebra in $\mathbf{D}(\Lambda)$ determined by the motivic homotopy Hopf algebra $\mathcal{H}_{\text {mot }}(k, \sigma ; \Lambda)$ via Proposition 1.4 is precisely the motivic Hopf algebra $\mathcal{H}_{\text {mot }}(k, \sigma ; \Lambda)$ as defined in [5, Définition 2.9(b)]. Similarly, for a motive $M \in \mathbf{D A}^{\text {ét }}(k ; \Lambda)$, the coaction of $\mathcal{H}_{\text {mot }}(k, \sigma ; \Lambda)$ on $\operatorname{Bti}_{\sigma}^{*}(M)$ given by [5, Proposition 2.11], is precisely the left coaction deduced from the homotopy comodule $\mathrm{Bti}_{\sigma}^{*}(M)$ via Proposition 1.7 .

Remark 8.11 - Keep the notation as in Definition 8.9. There is a functor

$$
\underline{\mathrm{Bti}}_{\sigma}^{*}: \mathbf{D M}^{\mathrm{ét}}(k ; \Lambda) \longrightarrow \operatorname{hocoMod}\left(\underline{\mathcal{H}}_{\text {mot }}(k, \sigma ; \Lambda)\right)
$$

which admits a simpler description than (31). Indeed, as in Definition 4.25 and Remark 4.26. $\mathbf{D M}^{\text {ét }}(k ; \Lambda)$ is the homotopy category of

$$
\mathbf{S p t}_{T_{k}^{\operatorname{tr}}}^{\sharp}(\mathbf{C p l}(\mathbf{P S t r}(\operatorname{Sm} / k ; \Lambda)))
$$

endowed with its stable ( $\mathbb{A}^{1}$, ét)-local structure. (Above, $\operatorname{PStr}(\operatorname{Sm} / k ; \Lambda)$ denotes the category of presheaves with transfers on $\mathrm{Sm} / k$ and $T_{k}^{\operatorname{tr}}=\Lambda_{\mathrm{tr}}\left(\mathbb{P}_{k}^{1}, \infty\right)$.) Now, given a commutative $T_{k}^{\mathrm{tr}}$-spectrum E,

$$
\Gamma\left(k ; \operatorname{Ev}_{0}\left({ }^{a} \widehat{\operatorname{Sing}_{\text {ét }}^{\mathbb{D}}}(\mathbf{E})\right)\right)
$$

is automatically a module over the cosimplicial algebra $\underline{\mathcal{H}}_{\text {mot }}(k, \sigma ; \Lambda)$ as defined in Definition 8.9. Moreover, if $\mathbf{E}$ is projectively stably $\left(\mathbb{A}^{1}\right.$, ét)-fibrant, $(33)$ is a 
homotopy comodule by Theorem 8.3 (B) applied to the commutative $T_{k}$-spectrum $\mathrm{O}_{\mathrm{tr}}(\mathbf{E})$ (obtained from $\mathbf{E}$ by forgetting transfers). In other word, 32 can be defined simply as the right derived functor

$$
\mathrm{R} \Gamma\left(k ; \operatorname{REv}_{0} \mathrm{R}\left({ }^{a} \widehat{\operatorname{Sing}}_{\text {ét }}^{\mathbb{D}}(-)\right)\right)
$$

on commutative $T_{k}^{\mathrm{tr}}$-spectra. On the contrary, the functor $(31)$ is defined as the composition of the right derived functor (34), which is then defined on commutative $T_{k}$-spectra, and the left derived endofunctor $\mathbf{S}^{\mathrm{tr}} \stackrel{\mathrm{L}}{\otimes}$-.

\section{REFERENCES}

1. Joseph Ayoub, Les six opérations de Grothendieck et le formalisme des cycles évanescents dans le monde motivique. I, Astérisque (2007), no. 314, $\mathrm{x}+466$ pp. (2008). MR 2423375 (2009h:14032)

2. L_ Les six opérations de Grothendieck et le formalisme des cycles évanescents dans le monde motivique. II, Astérisque (2007), no. 315, vi+364 pp. (2008). MR 2438151 (2009m:14007)

3. __ Note sur les opérations de Grothendieck et la réalisation de Betti, J. Inst. Math. Jussieu 9 (2010), no. 2, 225-263. MR 2602027 (2011c:14061)

4. __ La réalisation étale et les opérations de Grothendieck, Ann. Sci. Éc. Norm. Supér. (4) 47 (2014), no. 1, 1-145. MR 3205601

5 . __ L'algèbre de Hopf et le groupe de Galois motiviques d'un corps de caractéristique nulle, I, J. Reine Angew. Math. 693 (2014), 1-149. MR 3259031

6. L_ L'algèbre de Hopf et le groupe de Galois motiviques d'un corps de caractéristique nulle, II, J. Reine Angew. Math. 693 (2014), 151-226. MR 3259032

7. Utsav Choudhury and Martin Gallauer Alves de Souza, An isomorphism of motivic Galois groups, Preprint (2015), 54 pages.

8. Philip S. Hirschhorn, Model categories and their localizations, Mathematical Surveys and Monographs, vol. 99, American Mathematical Society, Providence, RI, 2003. MR 1944041 (2003j:18018)

9. Mark Hovey, Model categories, Mathematical Surveys and Monographs, vol. 63, American Mathematical Society, Providence, RI, 1999. MR 1650134 (99h:55031)

10. S S S Sectra and symmetric spectra in general model categories, J. Pure Appl. Algebra 165 (2001), no. 1, 63-127. MR 1860878 (2002j:55006)

11. Carlo Mazza, Vladimir Voevodsky, and Charles Weibel, Lecture notes on motivic cohomology, Clay Mathematics Monographs, vol. 2, American Mathematical Society, Providence, RI; Clay Mathematics Institute, Cambridge, MA, 2006. MR 2242284 (2007e:14035)

12. Madhav Nori, Lectures at TIFR, LaTeX notes by N. Fakhruddin, Unpublished (2000), 32 pages.

13. J. P. Pridham, Tannaka duality for enhanced triangulated categories, Preprint (2013), 52 pages.

14. Charles Rezk, A model for the homotopy theory of homotopy theory, Trans. Amer. Math. Soc. 353 (2001), no. 3, 973-1007 (electronic). MR 1804411 (2002a:55020)

15. Stefan Schwede and Brooke E. Shipley, Algebras and modules in monoidal model categories, Proc. London Math. Soc. (3) 80 (2000), no. 2, 491-511. MR 1734325 (2001c:18006)

16. Vladimir Voevodsky, Cancellation theorem, Doc. Math. (2010), no. Extra volume: Andrei A. Suslin sixtieth birthday, 671-685. MR 2804268 (2012d:14035)

17. Vladimir Voevodsky, Andrei Suslin, and Eric M. Friedlander, Cycles, transfers, and motivic homology theories, Annals of Mathematics Studies, vol. 143, Princeton University Press, Princeton, NJ, 2000. MR 1764197 (2001d:14026) 
Institut FÜr Mathematik, Universität ZÜRICH, WinterthuRerstr. 190, CH-8057 ZÜRICH, SWITZERLAND

\& CNRS, Laga Université Paris 13, 99 avenue J.B. Clément, 93430 Villetaneuse, FRANCE

E-mail address: joseph.ayoub@math.uzh.ch 